\title{
NURSING STUDENTS' EXPERIENCES OF MORAL UNCERTAINTY IN THE CONTEXT OF GLOBAL HEALTH PRACTICUMS
}

\author{
by \\ Madeleine Bernice Greig \\ BSN, The University of British Columbia (Okanagan), 2014 \\ A THESIS SUBMITTED IN PARTIAL FULFILLMENT OF \\ THE REQUIREMENTS FOR THE DEGREE OF \\ MASTER OF SCIENCE IN NURSING \\ in \\ THE COLLEGE OF GRADUATE STUDIES
}

THE UNIVERSITY OF BRITISH COLUMBIA

(Okanagan)

August 2018

(C) Madeleine Bernice Greig 
The following individuals certify that they have read, and recommend to the Faculty of Graduate and Postdoctoral Studies for acceptance, a thesis/dissertation entitled:

\section{NURSING STUDENTS' EXPERIENCES OF MORAL UNCERTAINTY IN THE CONTEXT OF GLOBAL HEALTH PRACTICUMS}

\begin{tabular}{ll} 
submitted by & Madeleine Bernice Greig in partial fulfillment of the requirements for \\
the degree of & Master of Science \\
\cline { 2 - 2 } & in
\end{tabular}

\section{Examining Committee:}

Dr. Barbara Pesut, Faculty of Health and Social Development

Supervisor

Dr. Patricia Marck, Faculty of Human and Social Development

Supervisory Committee Member

Dr. Michael Burgess, Faculty of Medicine

Supervisory Committee Member

Dr. Barbara Astle, School of Nursing

Additional Examiner 


\section{Abstract}

Background: Increasingly, undergraduate nursing students are electing to participate in global health practicums (GHPs). However, empirical and theoretical nursing literature that is dedicated to exploring the ethical implications of GHPs in nursing is surprisingly nascent.

Research Design: The aim of this interpretive descriptive study was to explore nursing students' experiences of moral uncertainty in the context of GHPs. Data was collected using semistructured interviews with 17 graduates of an undergraduate nursing program in Western Canada who had participated in a GHP in their final semester of nursing school.

Findings: Four main themes typifying the data were constructed. In the first theme, heightened reality in the global context, experiencing the 'exotic' nature of working in a foreign environment was a source of considerable moral uncertainty for past GHP participants. Moral uncertainty was born out of participants' grappling with issues related to heightened positions of power, influence, and visibility. In the second theme, cultural collisions in healthcare, moral uncertainty, and at times, moral distress, was derived from experiencing alternative approaches to care, and in particular, what participants perceived as a quelled, less action-oriented approach to care. In the third theme, blurring of boundaries: Scope of practice issues, practicing beyond one's scope of practice contributed substantially to participants' experiences of moral uncertainty. Lastly, in the fourth theme, connectedness, a sense of belonging was of paramount importance in helping participants to redress experiences of moral uncertainty.

Discussion: This study's findings indicate that nursing students from one Canadian university experienced a considerable amount of moral uncertainty, and less frequently, moral distress, during their GHP experience. Underlying much of the moral uncertainty was a misalignment between what participants expected their GHP to entail, and what was experienced in reality. 
Participants reflected deeply on these disparities, evidencing the development of a critical approach to global citizenship, as well as a heightened social consciousness. However, further integration of critical and transformative pedagogies into pre-departure training for GHPs, specifically, and undergraduate nursing education, more broadly, is needed. 


\section{Lay Summary}

More students than ever are electing to take part in international practicums from healthrelated disciplines. The purpose of this study was to explore the more challenging aspects of global health practicums (GHPs) attended by undergraduate nursing students from a Canadian university. In particular, this study explored nursing students' experiences of moral uncertainty during their GHP experience. Findings indicate that nursing students experienced considerable amounts of moral uncertainty during their GHP. Most often, participants' experiences of uncertainty stemmed from encountering different approaches to healthcare, being situated in new and 'exotic' cultural and clinical care environments, and when participants grappled with how best to stay within one's professional scope of practice. Of particular interest is the way in which participants' processed feelings of moral uncertainty, highlighting the need for further training to help nursing students navigate feelings of moral uncertainty in the context of GHPs. 


\section{Preface}

This thesis is original, unpublished, independent work by the author, Madeleine Greig. This study was granted ethical approval by the University of British Columbia Behavioural Research Ethics Board - Certificate Number: H16-01184. 
Table of Contents

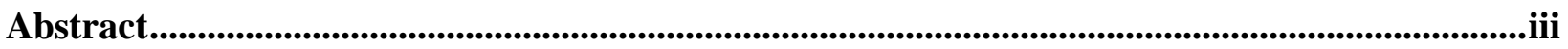

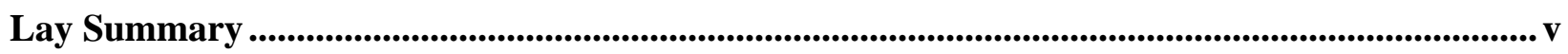

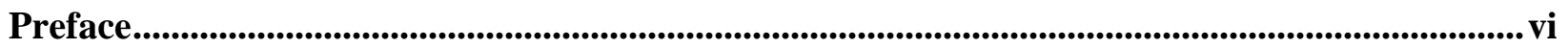

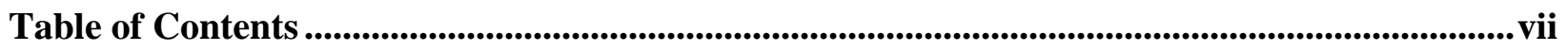

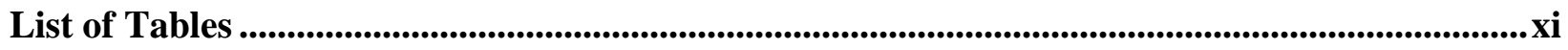

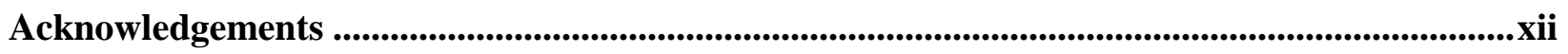

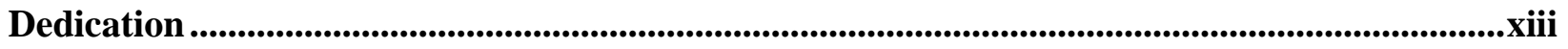

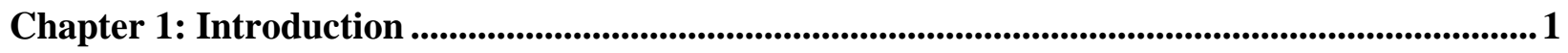

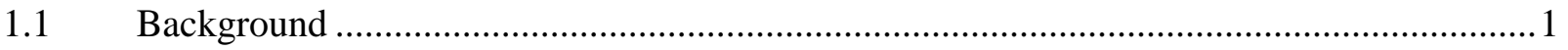

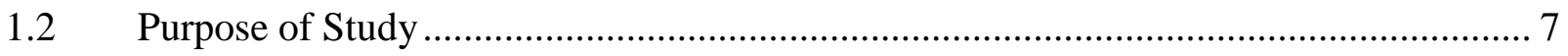

1.3 Definition of the GHP facilitated by UBC Okanagan............................ 8

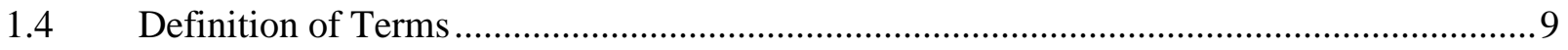

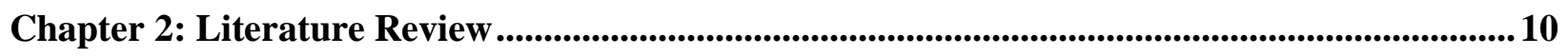

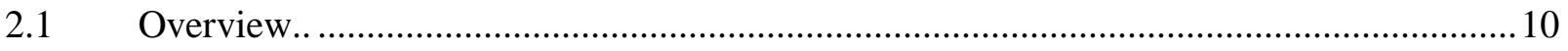

2.2 Methods for Scoping Review....................................................................... 10

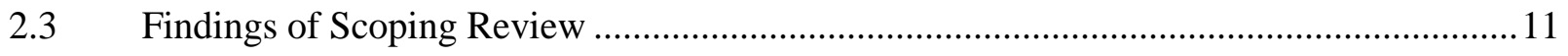

2.3.1 Creation and application of ethical guidelines................................................. 12

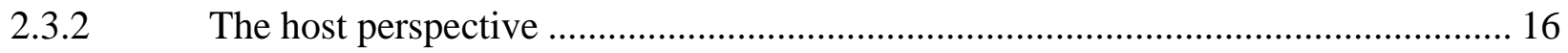

2.3.3 Health care professionals' and trainees' perceptions of GHPs ............................. 19

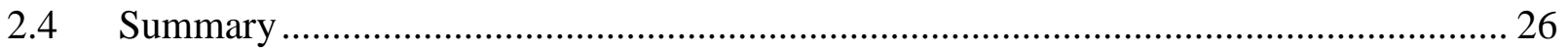

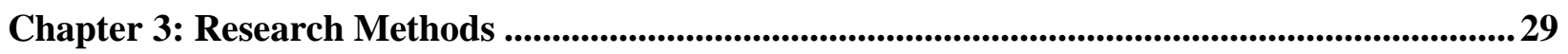

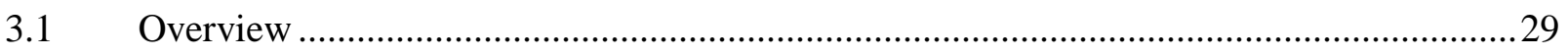




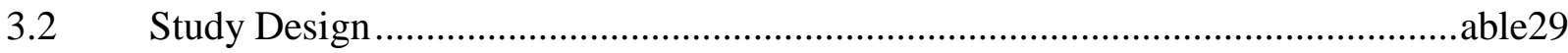

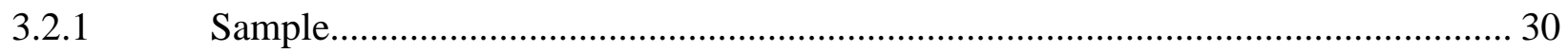

3.2.1.1 Sampling ........................................................................................... 30

3.2.1.2 Criterion for sample selection ...................................................................... 31

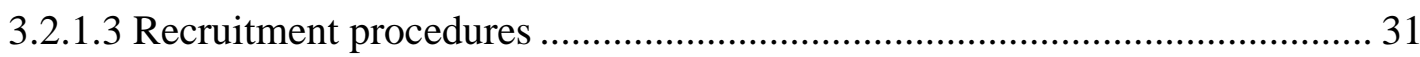

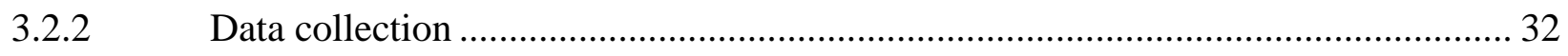

3.2.2.1 Semi-structured interviewing...................................................................... 33

3.2.2.2 Creation of the semi-structured interview guide............................................ 33

3.2.2.3 Interview process ……………………………...................................... 34

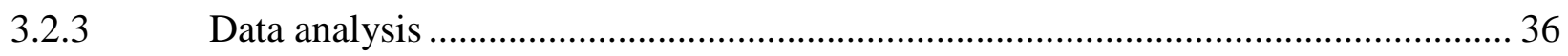

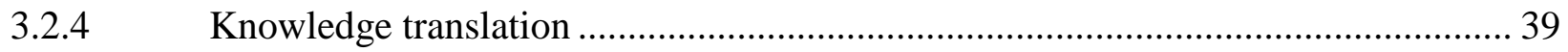

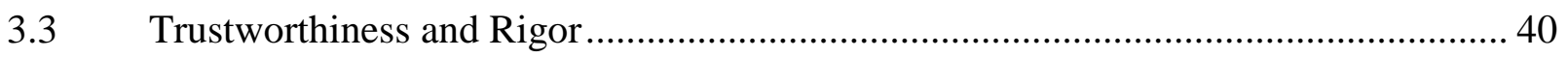

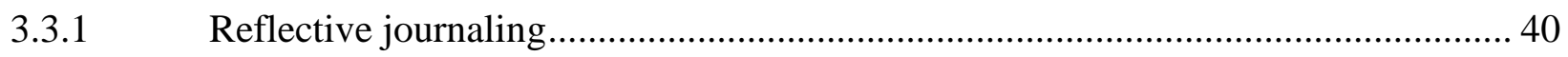

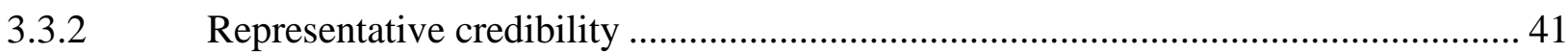

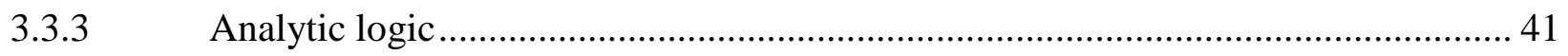

3.3.4 Interpretive authority ..................................................................................... 41

3.4 Research Ethics Review and Approval..................................................................... 42

Chapter 4: Findings .............................................................................................................................................. 44

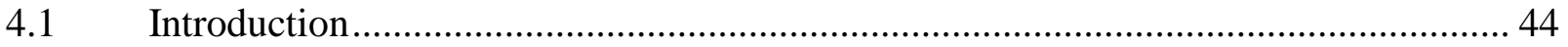

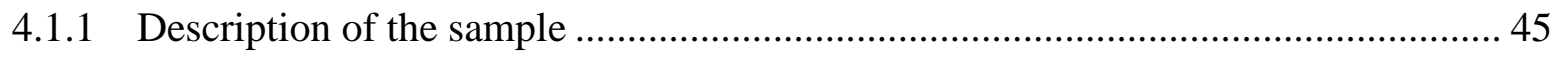

4.2 Heightened Reality in the Global Context .................................................................. 46

4.2.1 Heightened presence: "On display" in the global context ......................................... 47

4.2.2 Confronting issues of power and privilege ............................................................ 48

4.3 Cultural Collisions in Healthcare.............................................................................. 50 
4.3.1 A quelled approach to care: Experiences of moral uncertainty ............................ 51

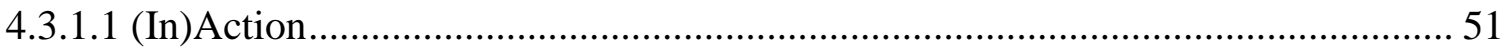

4.3.1.2 Relational Practice ............................................................................... 55

4.3.1.3 "It’s just a different type of care: Seeking clarity amidst moral uncertainty 56

4.3.1.4 "My hands were tied": Experiences of moral distress ............................... 58

4.4 Blurring of Boundaries: Scope of Practice Issues............................................... 60

4.4.1 Contextual factors: Laying the foundation for moral uncertainty ........................ 60

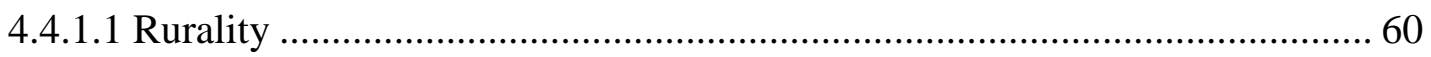

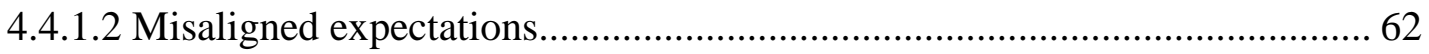

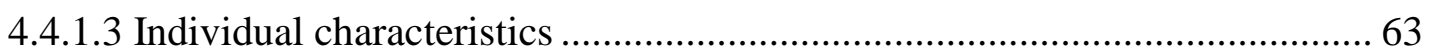

4.4.2 Experiences of moral uncertainty derived from issues of scope of practice............ 64

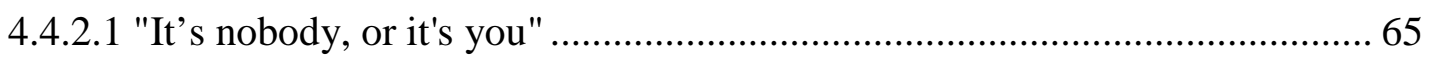

4.4.2.2 "I'll just watch for my own learning": Experiences of guilt and burden....... 68

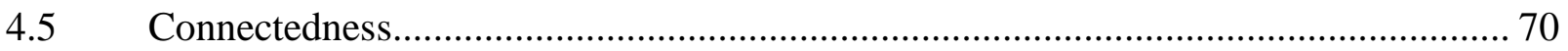

4.5.1 Something familiar: Working through experiences of moral uncertainty ............... 73

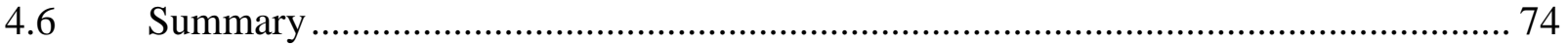

Chapter 5: Discussion of Findings, Recommendations, and Conclusions ...................................... 76

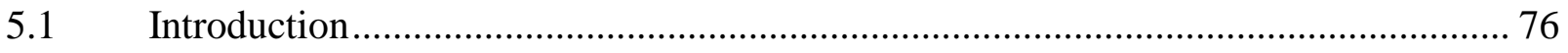

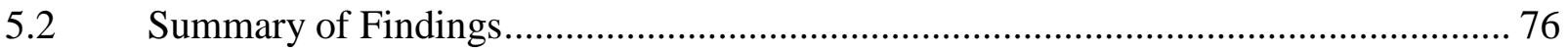

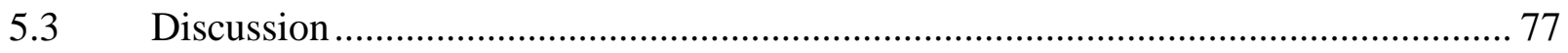

5.3.1 The role of expectations in nurses' experiences of moral uncertainty: What does the

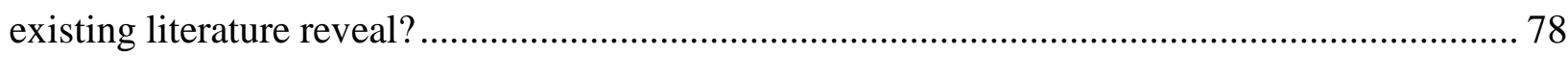

5.3.2 The role of expectations in nursing students' experiences of moral uncertainty during GHPs. 80 
5.3.2.1 Nursing students' motivations and expectations for engaging in GHPs ....... 80

5.3.2.2 The role of misaligned expectations in nursing students' experiences

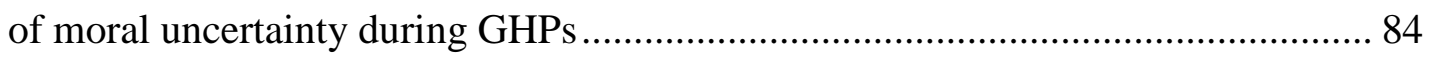

5.3.3 Redressing experiences of moral uncertainty in the context of GHPs.................... 87

5.3.4 Moral uncertainty: An opportunity to facilitate the development of students'

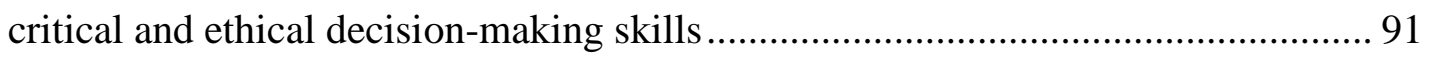

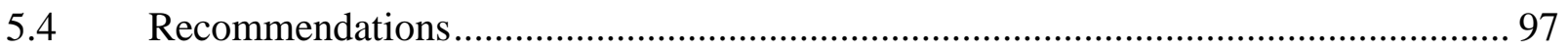

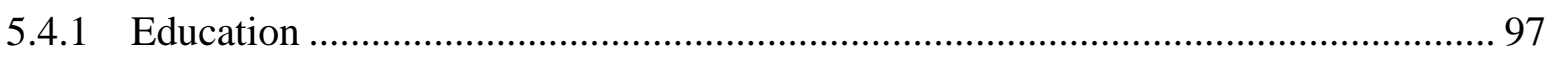

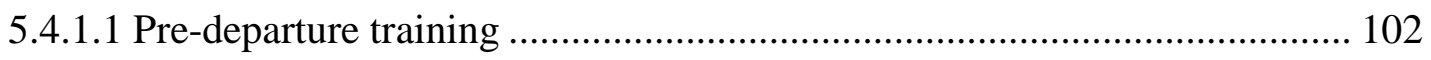

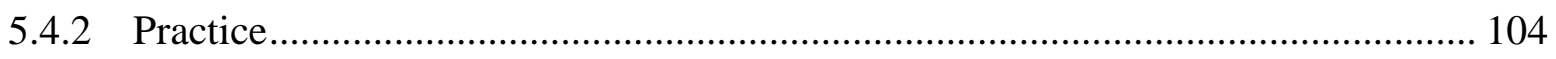

5.4.2.1 Clarifying program objectives ......................................................... 104

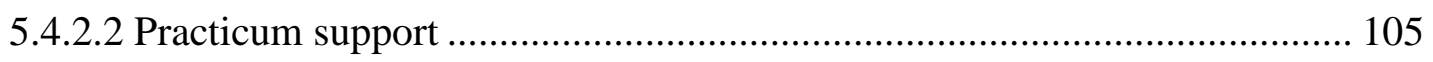

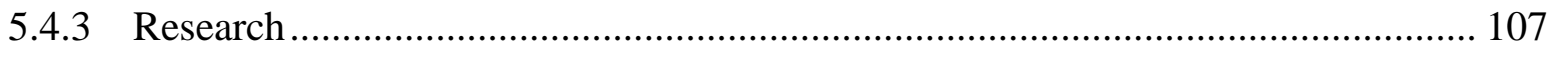

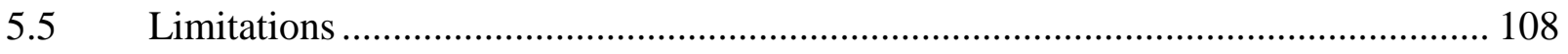

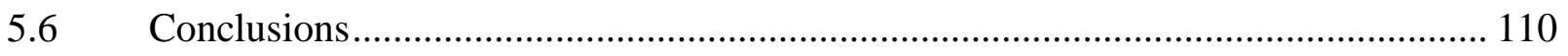

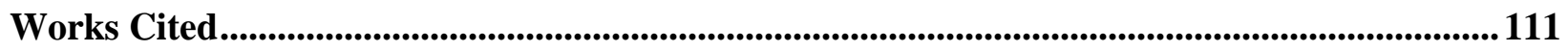

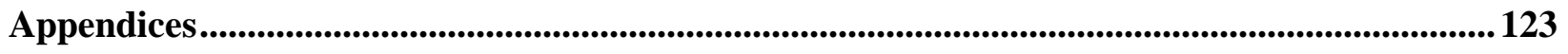

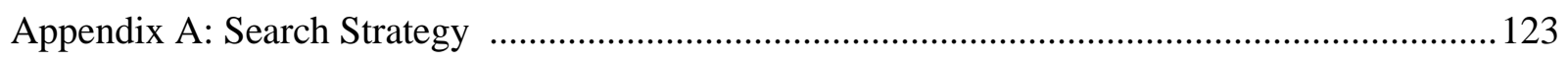

Appendix B: PRISMA Flow Diagram .......................................................................... 124

Appendix C: Recruitment Bulletin ............................................................................... 125

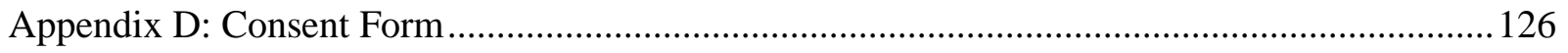

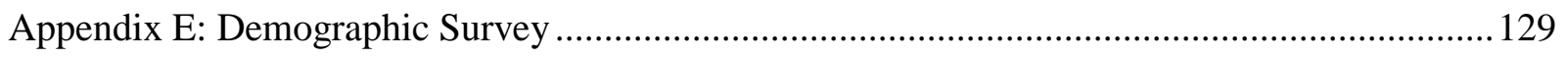

Appendix F: Semi-Structured Interview Guide .............................................................. 130

Appendix G: Field Notes Template .................................................................................. 132 


\section{List of Tables}

Table 1: Countries of studies' origins.............................................11

Table 2: Thematic accounts of moral uncertainty...................................45

Table 3: 'Soft' versus 'critical' global citizenship education............................100 


\section{Acknowledgements}

I offer my profound gratitude to all those faculty members, staff, and fellow students at UBC whom I have had the pleasure of learning from during my time in this warm and vibrant academic community.

This thesis would not have been possible without the sustained support and encouragement of a number of individuals.

Firstly, I must thank my supervisor, Barb Pesut, for her unparalleled mentorship, support, and friendship. Barb, you truly embody what it means to be an exceptional researcher, educator, and community member. I am so fortunate to have spent these past few years alongside you.

Secondly, to my supervisory committee, Patricia Marck and Michael Burgess; I am so thankful for your encouragement, thoughtfulness, and dedication to mentorship. Your feedback, throughout the entirety of this process, has been invaluable.

Thirdly, to all those who inhabit the Health, Ethics and Diversity Lab; you make work enjoyable.

Lastly, I must thank my family and friends who have provided me with unfaltering levels love and encouragement. I am profoundly grateful for you all. 


\section{Dedication}

Earth who gives to us this food.

Sun who makes it ripe and good.

Dear Sun, dear Earth by you we live,

Our loving thanks to you we give!

This thesis is dedicated to the Triumvirate of Farrell Street: Charlie, Bernice, \& Hector. 


\section{Chapter 1. Introduction}

\subsection{Background}

More health sciences students than ever are electing to participate in global health practicums (GHPs). Medical students have predominantly been the catalysts in the growth of these programs (Hanson, Harms, \& Plamondon, 2011; Izadnegahdar et al. 2008); however, the demand for GHPs in nursing has similarly increased (Burgess, Reimer-Kirkham, \& Astle, 2014; Battat et al., 2010; Reimer-Kirkham, Van Hofwegen, \& Pankratz, 2009; Hoe-Harwood, ReimerKirkham, Sawatzky, Terblanche, \& Van Hofwegen, 2009). In an exploration of the prevalence of 'innovative’ clinical placements offered at baccalaureate nursing programs in Canada, HoeHarwood et al. (2009) found that 54\% of nursing programs in Canada offered the opportunity to participate in an international clinical experience. Similar rates of engagement have been described in the populations comprised of medical students. For instance, in $2008,47 \%$ of the 129 accredited MD granting US medical schools offered global health initiatives, including the opportunity to engage in a GHP (Crump \& Sugarman, 2008). While statistics detailing nursing students' involvement in GHPs are limited, and the existing rates of engagement are dated, Izadnegahdar et al. identified that one in five Canadian medical students had participated in a GHP (2008).

The steady rise in requests for global health content and experiences (Crump \& Sugarman, 2010; McCall \& Iltis, 2014) can be attributed to a number of factors. These include hastened rates of globalization (Crump \& Sugarman, 2010; Kent-Wilkinson, Dietrich Leurer, Luimes, Ferguson, \& Murray, 2015; Shah \& Wu, 2008) and migration, as well as an engaged and sustained commitment to health equity. For example, the World Health Organization’s (WHO) Millennium Development Goals (2000-2015) and Sustainable Development Goals (2015-2030), 
which have shaped, and continue to shape, "the way we think and talk about the world, shaping the international discourse and debate on development, and stimulating popular awareness of moral imperatives such as achieving gender equality and ending poverty and starvation” (WHO, 2015, p. 4).

Over the past decade, the existing literature has largely focused on exploring the benefits of GHPs. Particular attention was paid to how these experiences impact students from the sending institutions. Health sciences students who have participated in GHPs have shown a greater understanding of socioeconomic influences, are more likely to pursue a career in global health and have shown substantive insight into the complexity of delivering culturally safe care (Izadnegahdar et al., 2008; Kulbok, Mitchell, Glick, and Greiner, 2012; Leininger, 1998). Furthermore, students who participated in a GHP reported improved diagnostic capabilities, an enhanced desire to care for indigent and marginalized populations, aspirations to practice primary care in the future, and increased interests in humanitarianism (Crump \& Sugarman, 2010; Lahey, 2012). However, while these gains for students are important, and indeed, laudable, there are substantial ethical risks associated with these experiences (Lahey, 2012; Crump \& Sugarman, 2008; Hanson, Harms, \& Plamondon, 2011) that warrant further exploration.

From the onset of a student's decision to participate in a GHP, the ethical implications must be considered, questioned, and reflected upon. As Lahey (2012) points out, major tenets of biomedical ethics such as beneficence, non-maleficence, justice, and respect for autonomy apply in the context of GHPs, just as they inform much of our ethical decision-making in Western healthcare contexts. However, when students have not been adequately prepared for the complexity inherent in providing care in the international setting, these key tenets of biomedical ethics have the potential to be disrupted or breached when competing ethical demands, new 
cultural practices, or intriguing opportunities for clinical engagement are presented (Lahey, 2012). Although it is not inherently wrong for students to pursue self-satisfying endeavors, competing interests, such as the opportunity to diversify one’s resume (Lahey, 2012), or the chance to participate in previously unlearned clinical skills (Hanson et al., 2011; Shah \& Wu, 2008), have the potential to undermine 'the good' that could be achieved during GHPs when the program is structured in a way that puts the benefits of the visiting students, above the potential risks or benefits to the host communities.

Similar lines of argumentation call into question the extent to which justice can be achieved during GHPs (Hanson et al., 2011; Lahey 2012). For instance, while GHPs may be well positioned to encourage global citizenship (Burgess, Reimer-Kirkham, \& Astle, 2014), and embedded within that, social justice (Mill, Astle, Ogilvie, \& Gastaldo, 2010), Hanson et al. (2011) argue that until the root causes of these inequities are critically acknowledged and addressed, little may be done to tackle what is seen as a widening gap of social inequities in health. This was later echoed by Lahey, who posited that “...although justice is inarguably served when redressing health disparities, the societal injustices that give rise to health disparities in the first place can be overlooked by medical groups with inadequate time or training to understand the larger local political calculus” (2012, p. 212). For instance, it can be argued that ethical issues related to global justice, and in turn, health inequity, are founded in the deeply seeded roots of poverty (Pogge, 2001; Ryan-Krause, 2016). While much has been done to redress issues of global poverty (United Nations, 2017), in the context of short-term GHPs, time constraints (Lahey, 2012), a focus on individual health needs, and a lack of understanding of the ramifications of global poverty (Ryan-Krause, 2016), make it unlikely for this root cause of global injustice to be addressed. 
Beyond the tensions inherent in key tenets of biomedical ethics such as beneficence and justice, ethical challenges related to personal issues, cultural differences, sustainability, and resource allocation have also been identified in the literature dedicated to exploring the ethical implications of GHPs (Crump \& Sugarman, 2008, Crump \& Sugarman, 2010, Lahey, 2012; Logar, Harrison, \& Glass, 2015). Furthermore, notable attention has been placed on examining the professional challenges raised during GHPs, including issues pertaining to practicing beyond one’s scope of practice (Crump \& Sugarman, 2008; Lahey, 2012; Logar et al, 2015; Hanson et al., 2011; Shah \& Wu, 2008). While some authors argue that this ethical breach during overseas practicums is, at times, deliberate (Hanson et al., 2011), others argue that practicing beyond one's scope of practice may be an unintended consequence of being thrust into a resource-poor setting that is in dire need of skilled hands, notwithstanding of the provider's preparation or experience (Crump \& Sugarman, 2008; Logar et al., 2012). Irrespective of the intent, however, this complex and nuanced issue requires consideration to the situation that students are being put in as well as to the issues that arise in relation to justice, patient safety, and nonmaleficence (Crump \& Sugarman, 2010; Lahey, 2012; Logar et al., 2015). As articulated in the following anecdote, threats to patient care as well as to trainees' psychological wellbeing can result from breaches in scope of practice:

After finishing my first year of medical school, I participated in a mission trip to Mexico. Before flying to Mexico, I was not given any cultural, medical, or other training, nor could I speak Spanish. Upon arriving, I was assigned to a clinic where there were hundreds of patients but only one physician. I remember vividly seeing a frail 11-year-old boy with polyuria, polydipsia and nocturia. My lack of medical training limited my differential. With only a scattered history and no other tests, I told him to limit his caffeine intake and 
see if that helps. Thinking back, he could have had a urinary tract infection, any number of renal abnormalities, or worse, I sent him out without ruling out diabetic ketoacidosis. And while I was seeing patients by myself, other first year medical students were performing surgeries in the other clinics and later bragging about it. (Anonymous Medical Student, Personal Reflection in Shah \& Wu, 2008, p. 376)

Despite these potential harms, such programs are often "conceived on the basis of justice, beneficence, and the duty to assist” (Crump \& Sugarman, 2008, p. 1458). Indeed, when GHPs are grounded in culturally sensitive and mutually beneficial partnerships, they can be well suited to addressing issues related to health equity (Adams, Wagner, Nutt, \& Binagwaho, 2016; RyanKrause, 2016). However, with the increased number of health sciences students electing to take part in GHPs we must take into consideration the ethical implications of these experiences and seek to balance - to the greatest extent possible - the range of benefits and potential harms associated with GHPs.

In an attempt to redress the ethical risks of GHPs, a growing body of theoretical literature largely grounded in medicine - is dedicated to preparing students for the types of ethical issues that may arise in the context of GHPs. For instance, in an effort to set the stage for program and curricular reform, a number of authors have discussed the spectrum of benefits and risks that have been associated with GHPs (Crump \& Sugarman, 2008; Lahey, 2012; Pinto \& Upshur, 2009; Shah \& Wu, 2008). Such discussions resulted in the creation of an ethics curriculum for global health outreach programs (Lahey, 2012), an ethics and best practice guideline for training experiences in global health (Crump \& Sugarman, 2010), a global health ethics framework (Pinto \& Upshur, 2009), and a call for an added emphasis on professionalism in global health curricula (Shah \& Wu, 2008). Furthermore, in a unique contribution to this body of literature, 
Logar et al. (2015) recommended that more ‘traditional global health ethics curricula” (p. 76) should include simulation-based learning as a means to better preparing medical students - and others - for the types of ethical issues they may encounter during GHPs (2015).

In comparison to the body of literature originating from the field of medicine, theoretical literature dedicated to examining the ethical implications of GHPs from a nursing perspective is limited, albeit insightful. Racine and Perron’s (2012) postcolonial analysis of GHPs examined the risks of reinforcing colonial behaviours when nursing students engaged in clinical practice overseas. The authors argued that concepts such as racialization - the "exclusionary process of social stratification that creates Otherness” (Racine \& Perron, 2012, p. 193) - and cultural voyeurism - "the exotic view of the Other" (Racine \& Perron, 2012, p. 192) -, pose significant challenges in developing GHPs that promote cultural safety. They concluded that it is not until a critical social consciousness is raised in nurse educators and nursing students, that GHPs will become little more than exploitive cultural excursions (Racine \& Perron, 2012). Along similar lines of critique, Leininger (1998) found that without care and attention to key aspects of transcultural learning, such as acknowledging inherent cultural biases and entering "the others' worlds as much as possible” (Leininger, 1998, p. 62), GHPs may be little more than opportunities that foster cultural imposition. Leininger (1998) found that a lack of cultural and contextual insight into the strengths of local care practices, coupled by a desire to change these “cultural lifeways” (Leininger, 1998, p. 59), fostered host faculty’s frustrations in hosting GHPs (Leininger, 1998). Lastly, in an avocation for adequately preparing trainees for GHPs, Levi (2009) emphasized the importance of incorporating cultural humility into pre-departure training. Levi (2009) argued that until biases such as those which characterize other cultures and care practices as inferior are identified and reflected upon, nursing students will be unable to enter 
into partnerships with the host communities that are built on principles of equity and respect (Levi, 2009).

\subsection{Purpose of Study}

The relative paucity of literature specific to the ethical implications of GHPs in nursing is troubling. With a few notable expectations (Burgess, Reimer-Kirkham, \& Astle, 2014; ReimerKirkham, Van Hofwegen, \& Pankratz, 2009), the nursing literature to date has focused primarily on identifying the benefits of GHPs as they pertain to nursing students and their future clientele. Central to many of the nursing specific studies was the idea that nursing students came away from GHPs with some element of psychological, cultural, social or professional growth (Afriyie Asenso, Reimer-Kirkham, \& Astle, 2013; Bentley \& Ellison, 2007, Bohman \& Borglin, 2014; Kollar \& Ailinger, 2002; Levine \& Perpetua, 2006; Sloand et al., 2008). For instance, GHPs have shown to contribute tremendously to fostering cultural competency in nursing students (Bentley \& Ellison, 2007; Carpenter \& Garcia, 2012; Kollar \& Ailinger, 2002; Larson et al., 2010; Levine \& Perpetua, 2006; Ruddock \& Turner, 2007), as well as facilitating “new ways of viewing the world” (Reimer-Kirkham, Van Hofwegen, \& Pankratz, 2009, p.5).

While these advancements in nursing students' professional and personal skill-sets are undoubtedly positive, the ethical implications of nursing students' involvement in GHPs have been largely overlooked. With the increased number of nursing students electing to participate in GHPs (Leininger, 1998; Reimer Kirkham et al., 2005), we must take into consideration the ethics of international training opportunities for undergraduate nursing students and avoid experiences whereby one partner potentially benefits at the expense and exploitation of the other (Crump \& Sugarman, 2008; Hanson et al., 2011; Lahey, 2012; Logar et al., 2015). 
Therefore, the purpose of this study is to explore the moral and ethical implications of GHPs attended by undergraduate nursing students from one university in Western Canada, through an examination of the moral uncertainty nursing students experience during GHPs. It is expected that this research will provide us with a better understanding of the moral challenges nursing students experience during GHPs, thus contributing to our ability to better prepare students for the moral and ethical complexities that are inherent in cross-cultural exchanges.

\subsection{Description of the GHP facilitated by UBC Okanagan}

UBC Okanagan’s School of Nursing offers fourth year nursing students with the opportunity to participate in a GHP in either Ghana, Africa, or Zambia, Africa. In order to participate in a GHP, students must meet a number of eligibility criteria; including, but not limited to, a Grade Point Average above 72\%, an exemplary attendance record in practice related courses, and no record of unprofessional conduct (Go Global, 2017). The GHP itself is fiveweeks long. The first week is dedicated to focused pre-departure seminars and labs (in Canada) and approximately three days of travel time. Weeks two through five occur in country and consist of practical experiences in diverse settings, such as "rural and remote community health clinics, government funded health centers, school and university settings, and a number of nongovernment organizations” (Go Global, 2017, para 2). GHPs facilitated by UBC Okanagan typically take place in early March through to mid-April. Students are accompanied on their GHP by one or two nursing instructors employed by UBC Okanagan’s School of Nursing. Supervision is varied given the geographic diversity of the student placements, as well as the ratio of students to instructors. However, continuous lines of communication between the GHP students and the instructors are maintained - to the greatest extent possible - throughout the course of the practicum (i.e. via telephone). 


\subsection{Definition of Terms}

Global health. For the purposes of this study, "global health is an area for study, research, and practice that places a priority on improving health and achieving equity in health for all people worldwide. Global health emphasizes transnational health issues, determinants, and solutions; involves many disciplines within and beyond the health sciences and promotes interdisciplinary collaboration; and is a synthesis of population-based prevention with individual-level clinical care” (Koplan et al., 2009, p. 1995).

Global health practicum (GHP). For the purposes of this study, global health practicum (GHP) will be used synonymously with the terms international medical elective, international surgical elective, nursing education exchange, international service learning, global health training program, and international education exchange.

Moral uncertainty. For the purposes of this study, moral uncertainty occurs when "a nurse feels indecision or a lack of clarity or is unable to even know what the moral problem is, while at the same time feeling uneasy or uncomfortable” (CNA, 2008, p. 6).

Global North. For the purposes of this study, Global North is defined as "those countries found mainly, but not exclusively, in the northern hemisphere, characterized by high levels of economic development” (Castree, Kitchin, \& Rogers, 2013).

Global South. For the purposes of this study, Global South is defined as “developing-countries, which are located primarily in the Southern Hemisphere (United Nations Development Programme, 2007, p. 1). 


\section{Chapter 2. Literature Review}

\subsection{Overview}

To date, theoretical and empirical work dedicated to examining the ethics of international practicums is surprisingly nascent (Crump \& Sugarman, 2010). This relative paucity of literature is further evidenced within the professional divides of the health sciences. The vast majority of literature specific to addressing the ethical implications of GHPs has originated from the field of medicine. Contributions from nursing and the allied-health sciences, while present, have been much more infrequent. As a result, this scoping review will take a multidisciplinary approach, and will include empirical evidence generated from the fields of medicine, nursing, and alliedhealth. The purpose of this scoping review is to examine the empirical literature specific to the ethical implications of GHPs.

\subsection{Methods for Scoping Review}

A scoping review of the literature published up to February of 2016 was conducted in CINAHL, MEDLINE, and EMBASE online databases. This initial search was repeated in May of 2018 to identify relevant articles $(n=3)$ that had been published in the time since the original search and screen had been conducted. At the time of the initial search, a cursory search of the terms "global health" and "international practicum" was employed, identifying vernacular that was specific to this field of study. From there, a combination of database specific controlled vocabulary and keyword searches were used. No limit to publishing date was applied. A detailed example of the search strategy used in Medline can be found in Appendix A. Searches resulted in the identification of 591 articles, which were then uploaded into RefWorks (Proquest, 2018) for storage and screening. Upon removal of duplicates, remaining articles were screened first on title and abstract and then assessed for eligibility based on full-text (see Appendix B). Reference lists 
of included articles were screened for additional relevancy, resulting in the identification of two additional studies. Inclusion criteria were empirical (both quantitative and qualitative) articles, written in the English language, with a focus on the ethical implications of GHPs. Articles were excluded if they were not specific to healthcare or if the full-text documents were not available through UBC's online databases.

\subsection{Findings of Scoping Review}

Twenty-one studies were screened in and grouped into three over-arching themes identified by the author: creation and application of ethical guidelines $(n=5)$, the host perspective $(n=6)$, and healthcare professionals’ and trainees’ perceptions of global health practicums $(n=10)$. Studies originated primarily from the United States and Canada; however, authorship was also representative of the United Kingdom and Uganda (see: Table 1.). Fourteen studies used qualitative methods, four studies used quantitative methods, two studies used mixed-methods, and one study was a literature review.

Table 1. Countries of studies’ origins

\section{Country}

United States

Canada

\section{United Kingdom}

Uganda

\section{Number of studies, n (\%)}

$12(57.1 \%)$

$6(28.6 \%)$

$2(9.5 \%)$

$1(4.8 \%)$ 


\subsubsection{Creation and application of ethical guidelines}

Five studies looked at the development and use of ethical guidelines to better prepare students for the types of ethical challenges that may arise in the global health context (DeCamp, Rodriguez, Hecht, Barry, \& Sugarman, 2013; Martin et al., 2013; Seymour, Benzian, \& Kalenderian, 2012; Wallace \& Webb, 2014; Zaidi, Haddad, \& Lathrop, 2015).

Three of the five articles focused on the development of ethics training programs (Decamp et al., 2013; Martin et al., 2013; Seymour et al., 2012). Martin et al. concentrated on gaining insight into the types of ethical conflicts medical residents most frequently encountered during both short-term surgical GHPs and domestic clerkships (2013). Through the analysis of survey data $(n=17)$, the authors found that when students engaged in international clerkships, they were more frequently exposed to ethical topics such as professional obligations of surgeons $(\mathrm{P}=$ 0.025) and truth-telling/surgeon patient relationships $(\mathrm{P}=0.044)$ (Martin et al., 2013). Surgical residents struggled to maintain professional obligations when delivering care under the constraints of short-term GHPs. As one participant noted, "I believe it is unethical to perform a procedure or provide treatment, then leave without ensuring adequate medical expertise remains to deal with any complications that arise...” (Martin et al., 2013, p. 369). Moreover, residents felt challenged by local cultural practices and language barriers to adequately satisfy the requirements of informed consent. Residents were unsure if patients fully understood the risks of surgery and whether or not they were aware of the critical role residents would be playing in their respective surgeries. While a small sample size limits the generalizability of the findings, these distinctions provide a starting point for tailoring ethics curricula for GHPs with a greater emphasis needing to be placed on topics such as the role of the trainee, cultural competence, 
appropriate postoperative follow-up, healthcare rationing, and the weight of Western norms (e.g., informed consent; confidentiality) in culturally distinct environments (Martin et al., 2013).

Seymour et al., (2012) in conjunction with the Harvard School of Dental Medicine, designed and piloted a Workshop for Ethical Volunteering in Global Health. The workshop focused on educating students about the difference between voluntourism - "a combination of volunteering and tourism” (Seymour et al., 2012, p. 1253)” - and responsible, and subsequently, more ethical, engagement during GHPs. Ultimately, the workshop sought to "provide students with a systematic approach to ethical volunteering, providing ways for them to critically reflect on motivations and attitudes related to traditional models of volunteering and facilitating alignment [of students' values] with principles of global health” (p. 1254). Participants ( $n=30)$ were asked to complete a survey prior to and upon completion of the workshop, rating the importance they placed on differing components of volunteering. Post workshop scores revealed that students placed more importance on components considered to be "the new standard for ethical volunteering in global health” (p. 1255) and less importance on more conventional components of volunteering when compared to pre-workshop scores. For example, greater importance was allotted to ideas such as cultural awareness and sustainability, rather than an emphasis on bringing donated medical supplies or simply providing clinical care (Seymour et al., 2012). The authors concluded by outlining the following guidelines for responsible volunteering: 1) think critically and reflect intensively before engaging in any volunteer activity, and be clear about motives and conscious of limitations; 2) engage only in activities that are relevant and sustainable in the host/recipient communities based on sound public health principles and evidence; 3) do as little harm as possible with any activity chosen; and 4) 
make sure that the volunteer activity benefits the community and is a challenging experience that leads to personal reflection and growth. (Seymour et al., 2012, p. 1256) While the results of this study are undoubtedly encouraging, several limitations do exist. Firstly, the small sample size $(n=30)$ limits the generalizability of the study's findings. Secondly, further research is needed to measure the translatability of the knowledge gleaned through this workshop into real-life volunteering experiences; a limitation that was acknowledged by the authors.

DeCamp et al., (2013) developed and evaluated a global health ethics curriculum entitled Ethical Challenges in Short-Term Global Health Training. In this comprehensive study, the authors solicited input into the types of ethical scenarios to include in an ethics curriculum, developed a freely available, online case curriculum, and evaluated the use of the program through the analysis of web usage data. Unfortunately, however, input was largely solicited from individuals in the Global North, with a self-admitted failure to adequately engage the perspectives of those in the Global South. Nevertheless, the case-based curriculum, which focused primarily on helping trainees to mitigate common ethical issues encountered during GHPs, was categorized into three overarching domains: Trainee Behaviour; Broader Context of Short-term Programs; and Research (DeCamp et al., 2013). Amongst other compelling topics, issues related to sustainability, trainee's scope of practice, developing cultural sensitivity, and identifying burdens of GHPs were addressed in the online ethics curriculum. Results from the analysis of user surveys and web usage data revealed a need for ethics training amongst shortterm global health trainees. Twenty-four percent of users reported they were required to take the curriculum in order to fulfill pre-departure requirements. Yet interestingly, of the nearly twothirds of identified users who had previously worked abroad or attended a GHP, only 52\% ( $n=$ 
156) of these individuals reported receiving training in global health ethics (DeCamp et al., 2013).

An additional two articles focused on the use of ethical frameworks to guide and evaluate GHPs (Wallace \& Webb, 2014; Zaidi et al., 2015). Zaidi and colleagues (2015) applied the ethical lens suggested by the Working Group on Ethics Guidelines for Global Health Training (WEIGHT) to 19 Obstetrics and Gynecology GHPs based out of the United States. Interviews with key members of these GHPs $(n=19)$ - all of whom represented perspectives from the Global North - revealed that varied techniques were used to address issues such as reciprocity, selection and training of participants, and assessing host community needs. However, while efforts were made to develop more mutually beneficial partnerships, significant gaps were identified. The majority of programs lacked application requirements for students wishing to train abroad; $63 \%$ of programs reported a single day of pre-departure training; $47 \%$ of programs interviewed did not perform formal needs assessments of the host community; and finally, for many, clinical care was deemed the "sole crux of their reciprocal relationship" (p. 1199). Yet interestingly, 32\% of programs maintained that they had not encountered any concerning ethical situations or questions - a statistic that merits further exploration (Zaidi et al., 2015).

Wallace and Webb (2014) conducted a limited, albeit insightful, review of the literature describing existing pre-departure training programs for medical students and found a relative scarcity of information. The authors argued that substantive pre-departure training with a focus on ethics would better prepare students to navigate the ethical dilemmas encountered during GHPs. While the authors did not elaborate on the screening process used, or the exact number of articles included in the review, they found that pre-departure training programs for existing GHPs were heavily located within the biomedical paradigm. Pre-departure training focused on 
the pathophysiology of infectious diseases and ways to protect one's own health when practicing in the international setting. Furthermore, while culture, context, and social issues were discussed, superficial reflections seemed to dominate existing curriculums. Lastly, references to historical influences of inequities in global health, and critical perspectives on the weight that power and privilege hold in international development were found to be lacking (Wallace \& Webb, 2014).

\subsubsection{The host perspective}

Articles within this theme explored perceptions and experiences of GHPs from various host perspectives. Four studies explored the perceptions held by healthcare professionals in communities that hosted participants of GHPs (Bozinoff et al., 2014; Kraeker \& Chandler, 2013; Kumwenda, Dowell, Daniels, \& Merrylees, 2015; Lukolyo et al., 2016). One study explored the perceptions held by local medical students (Elobu et al., 2014), and a final exploration by DeCamp, Enumah, O’Neil, and Sugarman (2014) provided insight into the perspectives of GHP care recipients.

The need for a more sustained commitment to equitable and mutually beneficial partnerships was a theme that resonated across the four studies that explored healthcare professionals’ perceptions of GHPs (Bozinoff et al., 2014; Kraeker \& Chandler, 2013; Kumwenda et al., 2015). Bozinoff et al.’s (2014) mixed-methods study identified that questions of reciprocity originated, in part, from a lack of formalized exchange programs. Specifically, host supervisors of medical electives indicated that local students - from low resource settings should be given the opportunity to participate in South - North partnerships, thereby complimenting the North - South exchange programs that dominate most GHP designs (Bozinoff et al., 2014). Moreover, Kraeker and Chandler (2013) found that healthcare professionals $(n=9)$ who frequently hosted GHPs at the University of Namibia's School of 
Medicine experienced a lack of collaboration with sending institutions. Findings suggested that visitors lacked a sense of contextual awareness and commitment to understanding local priorities, capabilities, and cultural practices. This led to feelings of frustration as time and energy were wasted (Kraeker \& Chandler, 2013). Similarly, Kumwenda et al.’s (2015) qualitative exploration found that improved collaboration between the sending and hosting institutions could strengthen existing GHPs. Local supervisors of GHP students $(n=14)$ emphasized the need for sending institutions and GHP students to take a more proactive stance in ensuring communities and individuals benefit from hosting GHPs. Host supervisors highlighted the need for clearly defined and mutually identified program objectives as a way to uphold the safety, ethics, and integrity of GHPs:

I think some of them [elective students] are frustrated because we will not allow them to do things that they are not qualified to do. This is not a bush hospital; this is a hospital that has been there for 105 years...We are never enough, but I would never put a scalpel in the hand of a student, never, it has never happened. (Kumwenda et al., 2015, p. 629) Lastly, Lukolyo et al. (2016) explored host clinical preceptors' expectations and perceptions of short-term medical practicums at four clinical sites in sub-Saharan Africa. Despite viewing these experiences as favourable, host clinical preceptors indicated that a greater emphasis should be placed on cultural sensitivity training, length of the practicum, and encouraging interprofessional teamwork to ensure that GHPs remain a mutually beneficial experience for all those involved (Lukolyo et al., 2016).

Notwithstanding these challenges, host partners in all four of these studies expressed a strong desire to continue hosting international colleagues and students (Bozinoff et al., 2014; Kraeker \& Chandler, 2013; Kumwenda et al., 2015; Lukolyo et al., 2016). This finding may be 
related to the relationships that existed between the host partners and the study authors. For instance, many of the medical elective supervisors interviewed by Bozinoff et al. (2014) previously held or at one time had sought to connect with the university responsible for conducting this research study. This power differential, evident in Bozinoff et al.’s (2014) study as well as in the research conducted by Kumwenda et al. (2015), Kraeker and Chandler (2013) and Lukolyo et al. (2016), has the potential to heighten response bias as the participants may not want to jeopardize any current or future working relationships with the institution conducting the research.

In an exploration of Ugandan anaesthesia trainees’ perceptions of international global health collaborations, Elobu et al.’s (2014) descriptive, cross-sectional survey $(n=33)$ revealed that the majority of Ugandan anesthesia trainees (75\%) benefited from hosting international colleagues. In particular, Ugandan trainees noted improvements to their clinical skills which were augmented through the delivery of skills workshops and specialty education. Given this finding, it is perhaps unsurprising to note that Ugandan trainees felt they learned more from visiting faculty, than visiting students; confirming once again the need for improved collaboration and presence from sending institutions. Furthermore, while trainees were supportive of international collaborations, substantive areas for improvement were identified. A considerable portion of respondents (31\%) reported a level of discomfort with the ethical decision making of visiting faculty members. Interestingly, this was mirrored by $30 \%$ of respondents reporting similar discomforts with decisions made by faculty from the host institution. Unfortunately, none of the respondents expanded on the topic of ethics in the space provided (Elobu et al., 2014). 
A final article reported on the perceptions of individuals who were receiving care from a short-term medical outreach program (DeCamp et al., 2014). Medical and nursing students were often part of these faith-based mission trips, which provided important insight into how care recipients respond to services provided by trainees. Participants $(n=30)$ were overwhelmingly positive about the care they received from students, describing involvement in three ways: "as beneficial to the student's professional development, as beneficial to the communities because of the care delivered, and as part of future non-specific benefits to the community” (DeCamp et al., 2014, p. 420).

While DeCamp et al.’s (2014) findings are indicative of a substantial achievement, limitations in interpreting the results of this study do exist. For instance, irrespective of interviewers' efforts to dissociate themselves from the medical outreach program, participants frequently misidentified the interviewers as members of the care team delivering services to the

community (DeCamp et al., 2014). This misidentification, which was readily acknowledged by the researchers, has the potential for heightening response bias, as participants may not feel comfortable expressing their true sentiments towards the medical program for fear of jeopardizing their current and prospective access to care (DeCamp et al., 2014).

\subsubsection{Healthcare professionals' and trainees' perceptions of GHPs}

Of the articles identified that explored healthcare professionals' and trainees' perceptions of GHPs, three articles explicitly sought to characterize and explore the ethical challenges encountered by students during GHPs (Dell et al., 2014; Elit et al., 2011; Harrison, Logar, Le, Glass, 2016). Elit et al.'s (2011) qualitative inquiry constructed five over-arching themes: (i) uncertainty about how best to help; (ii) perceptions of Western medical students as different; (iii) moving beyond one's scope of practice; (iv) navigating different cultures of medicine; and (v) 
unilateral capacity building (p. 704). The small, homogenous sample of students who were interviewed $(n=12)$ grappled with the level of responsibility that was allotted to them by local healthcare professionals and patients (Elit et al., 2011). On some accounts, students felt overwhelmed by the perception that they should be - and were - capable of performing skills beyond their training and ability. Some students questioned whether patient safety was compromised as a result of their involvement in clinical care. And yet, at other times, students felt restricted in their ability to advocate for patients within a healthcare context that was so different than their own (Elit et al., 2011).

Twenty-three Canadian medical trainees who had attended a GHP during medical school expressed similar sentiments. Comparable to Elit et al.’s study (2014), Dell and colleagues (2013) explored the ethical challenges faced by medical students during GHPs, with an added focus on examining scenarios where students' physical safety was jeopardized. Of the 23 individuals interviewed, 13 participants (57\%) remarked on either witnessing and/or being involved in an ethically challenging situation. Often centered on issues of resource disparity, students found that they struggled to provide care in an environment which lacked affordable access to healthcare services: "a lot of patients really needed tests or blood transfusions and could not afford them and therefore they were just left to just bleed to death” (Dell et al., 2013, p. 66). This was compounded by a lack of human resources in many clinical settings, resulting in some trainees having to manage patient loads that were beyond their clinical ability and scope of practice. Additionally, students felt conflicted by how little they were able to give back to the local community, frequently referencing the tendency of GHPs to be largely unilateral capacity building exercises: 
Although I took money and donated it to the hospitals and I did try and contribute, I basically told myself I would never go back to a developing country until I had something to offer, I felt like it was very one-sided, that all I did was take, take, take. (Research Participant, Dell et al., 2013, p. 67)

Moreover, Dell et al. (2013) found that 83\% ( $n=19)$ of participants experienced threats to personal safety during GHPs. These threats to participants’ psychological and physical wellbeing included exposure to infectious diseases, a lack of understanding of the local cultural and political climate, and diminished occupational health and safety standards. Furthermore, despite $60 \%$ of participants indicating that they participated in some element of pre-departure training, Dell et al. highlighted that participants still experienced a vast array of ethical challenges during their GHP experience (2013). Unfortunately, the type, length, and/or content of the pre-departure training was not reported on, limiting our ability to interpret the implied sense of inadequacy of pre-departure training.

Lastly, Harrison et al. (2016) explored multi-professional (e.g., medicine, nursing, pharmacy) perceptions of the ethical issues that are encountered by trainees during global health outreach work. Participants interviewed in this study "described ethical challenges that they had experienced and believed that trainees may also encounter when working in resource-limited settings" (Harrison et al., 2016, p. 6). Analysis of the focus group and interview data revealed a number of ethical dilemmas that had the potential to challenge trainees during overseas practicums. These ethical dilemmas were then constructed into four overarching themes related to: "(1) cultural differences (informed consent, truth-telling, autonomy); (2) professional issues (power dynamics, training of local staff, corruption); (3) limited resources (scope of practice, material shortages); (4) personal moral development (dealing with moral distress, establishing a 
moral compass, humility and self-awareness)” (Harrison et al., 2016, p. 1). While the views solicited only represented those of US-based healthcare professionals - a limitation acknowledged by the authors -, the themes identified have the potential to inform the training health sciences students receive prior to engaging in overseas practicums (Harrison et al., 2016).

Four articles explored the outcomes, complexities, and meaning of GHPs as perceived by medical students (Abedini, Gruppen, Kolar, \& Kumagai, 2012; Kumwenda, Royan, Ringsell \& Dowell, 2014; McCarthy, Petrosoniak, \& Varpio, 2013; Petrosoniak, McCarthy, \& Varpio, 2010). While these studies did not explicitly seek to identify the ethical implications of GHPs, questions attributed to the ethics of engaging in GHPs were raised.

To garner a better understanding of the goals and implications of GHPs, Petrosoniak et al. (2010) conducted a qualitative exploration of the harms and benefits of GHPs as experienced by medical students. Interviews, conducted with a convenience sample of medical trainees $(n=10)$ and healthcare professionals ( $n=10)$, suggested that existing GHPs are rife with elements of medical tourism - experiences which disproportionately favour the participants of the GHP over the host (Petrosoniak et al., 2010). In a similar exploration of the complexities inherent in GHPs, Abedini et al.'s qualitative (2012) study found that first-year medical students benefited greatly from international medical electives. While contextual elements of the study, such as the duration of the GHP, limit the transferability of the findings, $85 \%(n=11)$ of first-year medical students who participated in a one-week GHP reported an enhancement in language and clinical skills, as well as a heightened understanding of the complexities of working in a foreign healthcare system. Furthermore, an additional seven students (54\%) went on to say that they felt their clinical contributions positively impacted the host communities. Nevertheless, through reflexive discussions and probing by the interviewers, students remarked on the more 
challenging aspects of GHPs. Questioning of peers’ motivations for attending GHPs, awareness of existing power differentials, practicing outside of one's scope of practice, and the possible deleterious effects of short-term work were all highlighted by participants. One student's critical reflection encompassed many of these issues:

I think that it's a slippery slope for you [as a medical student] to subqualify yourself to go down...as an American “doctor”, sort of this omniscient being. That, I think, is dangerous, and from a public health standpoint if you go anywhere for a week or two and you do something, what are you really accomplishing? (Research Participant in Abedini et al., 2012, p. 823-824)

What is perhaps most interesting to note, however, is that prior to these interviews many of the students $(n=5)$ had not actively reflected on their international experience. This highlights the need for both extensive pre-departure and post-departure training (Abedini et al., 2012).

Providing an interesting comparison to the first-year medical students whose reflections were reported in Abedini et al.’s (2012) study, Kumwenda and colleagues (2014) found that students who were closer to finishing their medical degree, and who were generally older, reported greater satisfaction in feeling prepared for their GHP. Additionally, findings from their qualitative inquiry $(n=29)$ revealed that students appeared to be well prepared to navigate potentially challenging ethical encounters. Times where participants engaged in patient care that was beyond their clinical ability did occur $(n=2)$, however, these instances did not occur under deliberate attempts to practice inappropriately. Rather, they arose out of miscommunications between the local healthcare team and the students, whereby the students were mistaken for fully trained physicians (Kumwenda et al., 2014). In one particular scenario, Kumwenda et al. (2014) discovered that "local staff had mistaken the student for a new volunteer doctor and had expected 
that the 'expatriate' would take charge of the emergency at hand” (p. 48). This misconception illustrates the potentially deleterious effects of not fully orienting and introducing visiting students to the host community and prospective workplace (Kumwenda et al., 2014).

A final study sought to identify the complex relationships inherent in GHPs through an exploration of the perceptions held by the three main stakeholder groups involved in GHPs: participants (trainees and professionals), host communities, and sponsoring institutions (McCarthy et al., 2013). Interviews with previous participants of GHPs, as well as with healthcare professionals with backgrounds in global health $(n=20)$, revealed that considerable amounts of tension existed amongst the three key stakeholder groups. Student participants spoke of the challenges in upholding principles of cultural humility, and the difficulties that arose when objectives and expectations were misaligned between participants and host communities. Similar tensions existed between participants and sending institutions, particularly when program objectives were not well defined at the outset (McCarthy et al., 2013). Unfortunately, data was not collected from two of the three stakeholder groups - sending institutions and host communities - resulting in a limited ability to provide a comprehensive perspective of the tensions that exist amongst the three key stakeholder groups. This limitation was readily acknowledged by the authors (McCarthy et al., 2013).

Lastly, three articles addressed the ethical implications of GHPs from a nursing perspective (Caldwell \& Purtzer, 2014; Evanson \& Zust, 2006; Ryan-Krause, 2016). While none of these studies explicitly sought to identify the ethical implications of GHPs, insight into the long-term learning effects of international practicums revealed that nursing students are beginning to question the ethical consequences of GHPs. For instance, Evanson \& Zust (2006) found that while nursing students were positively impacted, both personally and professionally, 
by their GHP experience, they began to question whether the local Guatemalan community in which their GHP was based benefited at all from the experience. As one participant remarked: “...did we really even help? I mean, we helped by bringing the supplies, but for what? To sit in a closet. They’re afraid to use them” (p. 416). Caution must be taken, however, in interpreting the results of this study, as the transferability of these findings are limited by a small convenience sample $(n=6)$ of former undergraduate nursing students.

Similar results were obtained in Ryan-Krause’s (2016) exploration of the "essential lessons learned” (p. 379) from short-term GHPs involving advance practice nursing students from the United States. While analysis of nursing students' written personal reflections revealed improvements in clinical skills and linguistic abilities, ethical challenges related to "undermining the value of local providers, obstacles to appropriate follow-up care, and disruption of community life” (Ryan-Krause, 2016, p. 379) were also identified. Similar to the uncertainty reported in Evanson and Zust's (2006) qualitative exploration, Ryan-Krause (2016) found that nursing students questioned the impact of their presence in the local community:

I still feel ambivalent about my individual contribution. Perhaps this is the unavoidable nature of a global service trip. Working in a developing country is a reminder of the inherent power imbalances between rich and poor, patients and providers, the helped and the helpers. My hope is that awareness of these complex dynamics will ultimately enable me to maintain the right intentions and do more good in the world. (Ryan-Krause, 2016, p. 382)

Lastly, through the analysis of questionnaire data $(n=41)$, Caldwell and Purtzer (2014) identified that nursing students began to question whether their presence during a short-term GHP was a source of more harm, than good, to the local Honduran community in which their 
GHP was based. Responses to four open-ended questions found that students wrestled with questions pertaining to patient autonomy, sustainability, power and privilege, and the challenges that come with working with a marginalized population. Interestingly, however, the authors identified that participants did not possess the skills to adequately mitigate or process these ethical tensions. Thus, while the identification of these complexities may be indicative of achieving transformational learning among trainees, as Caldwell and Purtzer eloquently stated, “at whose expense?” (2014, p. 582).

\subsection{Summary}

As a result of the scoping nature of this review, limitations exist. Given the limited number of databases searched and the controlled vocabulary used, it is reasonable to expect that additional evidence exists beyond the literature identified for this review. Furthermore, upon critical reflection of the theoretical, assumptive, and methodological traditions that make up the empirical literature included in this review, certain limitations in considering the credibility of this body of literature do exist. For instance, while 14 of the 21 identified articles drew upon qualitative methods, none of the studies incorporated data collection methods beyond participant interviews or open text questionnaires. Future qualitative inquiries should look to incorporate data collection and analysis techniques that seek to contextualize the study findings, employing methods such as participant observation and grounding data analysis in ethical theories, models, and frameworks.

The identification of 21 empirical articles demonstrates a growing body of health sciences literature that is dedicated to researching the ethical implications of GHPs. Articles identified in this scoping review provide important insight into the creation and application of ethical guidelines; the host perspective; and healthcare professionals' and trainees' perceptions of GHPs. 
Significant advances have been made in identifying and seeking to redress the ethical implications of GHPs. Drawing on a variety of perspectives, numerous studies recognized the ethical tensions, dilemmas, and challenges that exist in GHPs (Abedini et al., 2012; Caldwell \& Purtzer, 2014; Dell et al., 2014; Elit et al., 2011; Evanson \& Zust, 2006; Kumwenda et al., 2014; McCarthy et al., 2013; Petrosoniak et al., 2010). The majority of studies within this realm focused on how these ethical challenges are perceived or experienced by health sciences students. However, an emergent body of evidence exists that is dedicated to gaining a more comprehensive picture of GHPs; amplifying the voices of host institutions, patients, and students whose critical input was discounted until 2013 (Bozinoff et al., 2014; DeCamp et al., 2014; Elobu et al., 2014; Kraeker \& Chandler, 2013; Kumwenda et al., 2015; Lukolyo et al., 2016; Ryan-Krause, 2016).

In addition to ethical challenges related to cultural collisions, sustainability, reciprocity, resource allocation, and professional obligation and ability, tensions within the main tenets of biomedical ethics were discussed. Students wrestled with situations that jeopardized patient autonomy and justice and began to question motivations and actions that threatened beneficence and non-maleficence. It is interesting to note, however, that while the identification of ethical challenges was a predominant theme, there was little exploration of why such ethical challenges arose. Future inquiry into why these ethical tensions exist and how these challenges play out among GHP stakeholders has the potential to supplement existing ethical guidelines and predeparture training.

Lastly, comparable to the professional divide that is seen in the theoretical literature, the majority of empirical evidence specific to the ethical implications of GHPs originates from the field of medicine. While much can be gleaned from the fields of medicine and allied-health, 
there exists a large void in the empirical literature dedicated to the ethical implications of GHPs in nursing. Beyond simply discussing the benefits of GHPs as they are accrued to participants from the sending institutions, it is time nurses and nursing scholars alike question the moral and ethical implications of GHPs and expand on those "unsettling feelings” (Evanson \& Zust, 2006, p. 416) that undoubtedly exist. As such, the purpose of this study is to examine some of the more challenging aspects of international nursing practicums, through an exploration of the moral uncertainty nursing students experience during GHPs. 


\section{Chapter 3. Research Methods}

\subsection{Overview}

The current empirical and theoretical nursing literature inadequately explores the significant ethical implications of GHPs in nursing. Specifically, this dearth of information is evidenced by a lack of knowledge on the ethical and moral challenges that nursing students experience during overseas practicums. Therefore, through methods aligned with interpretive description, this study sought to bridge this gap through an exploration of the moral uncertainty nursing students experienced during GHPs facilitated by one university in Western Canada.

In this chapter, the over-arching study design, data collection and analysis strategies, ethical considerations, and methods to achieving methodological trustworthiness and rigor will be discussed. An in-depth description of the sample will be presented in Chapter Four.

\subsection{Study Design}

This qualitative study was guided by interpretive description. Predicated on the assumptions of naturalistic inquiry, interpretive description is a "research design logic" (Thorne, 2008, p. 27) that holds particular relevancy for addressing issues and challenges that arise in practice, and in particular, within the nursing practice (Thorne, 2008). Interpretive description, Thorne (2008) argues,

...constitutes a method that...pushes one into the "field" in a logical, systematic and defensible manner, and creates the context in which engagement with the data extends the interpretive mind beyond the self-evident - including both the assumed knowledge and what has already been established - to see what else might be there” (p. 35).

In other words, this pragmatic approach to inquiry seeks to generate new understandings of a particular phenomenon of interest, through an interpretation of common themes, patterns, and 
relationships that exist within individuals' experiences of the topic of interest (Thorne, Reimer Kirkham, \& MacDonald-Emes, 1997). As the intent of this study was to generate a better understanding of the ethical implications of GHPs in nursing, and in particular, the type of moral uncertainty nursing students experienced during such excursions, the clinical focus of interpretive description (Thorne, 2008) aligned well with the objective of this study.

Ethical approval for this study was obtained from the Behavioural Research Ethics Board (BREB) at the University of British Columbia's Okanagan campus.

\subsubsection{Sample}

\subsubsection{Sampling}

As opposed to quantitative research designs, the intent of qualitative research, including interpretive descriptive studies, is never grounded in “claims to generalization” (Thorne, 2008, p. 36). Rather, the goal of qualitative research is to discover meaning (Creswell 2007; Thorne et al. 2004), and to be able to articulate this meaning through rich, expressive accounts of participants' experiences with a particular phenomenon of interest (Thorne et al., 2004). Therefore, in light of the subject matter which was explored in this study, and on understandings of the empirical evidence currently available on this topic, I sought to recruit 15-20 participants to participate in this research study.

A convenience sampling technique was used to recruit participants to this study. Selected for its accessible and feasible nature, convenience sampling - a type of non-random sampling was used to select participants who were easily accessible and at the convenience of the researcher (Sedgwick, 2013). Thorne (2008) posits that convenience samples are "an excellent source of insight” (p. 89) for elucidating descriptive aspects of the phenomenon of interest in the early stages of research. Interestingly, however, while the sample selected to participate in this 
study was derived from a convenience sampling technique, the participants who elected to take part in this study had a unique ability to speak to the more morally and/or ethically challenging aspects of GHPs. Thus, if purposive sampling is defined as an approach whereby individuals "are recruited by virtue of some angle of the experience that they might help us to better understand" (Thorne, 2008, p. 90), both convenience and purpose sampling techniques were used in the creation of this study's sample. Nevertheless, attention was given to the limitations associated with a convenience sample by allowing for additional sampling methods to be drawn upon should the need arise (e.g., theoretical sampling). However, due to a rapid response rate and the variable characteristics (e.g., year of GHP attended) of volunteering participants, the need for additional sampling methods did not arise in the context of this study.

\subsubsection{Criterion for sample selection}

Eligibility criteria required that participants were graduates of the undergraduate nursing program at UBC's Okanagan campus who participated in a GHP in their final semester of nursing school between the years of 2011 and 2016, inclusive. As a result of the early exploratory nature of this research, and in keeping with a scope of study that is manageable at a master's level, graduates who attended GHPs that were not organized by UBC's Okanagan campus were excluded from partaking in this study.

\subsubsection{Recruitment procedures}

Recruitment for this study occurred via recruitment bulletins (Appendix C), which were distributed by e-mail to graduates of UBC Okanagan's School of Nursing who previously attended a GHP during their undergraduate nursing degree. E-mails were sent out exclusively to nursing students who participated in a GHP between the years of 2011 and 2016, inclusive. Access to this list of potential participants was obtained through the School of Nursing's 
practicum placement coordinator, upon approval by the Behavioural Research Ethics Board at UBC’s Okanagan campus.

Upon receipt of the recruitment e-mail, interested individuals were asked to contact me either by phone or e-mail should they wish to learn more about the research objective and the possibility of participating in this research study. I then followed up with interested individuals at their earliest convenience, either by phone or by e-mail. Follow-up sessions were used to further explain the purpose of the study, to screen for eligibility, and to answer any questions that the interested individual might have. At the closing of these follow-up sessions, consent forms (Appendix D) were presented to eligible individuals via e-mail who indicated that they were interested to participating in this research study. Participants were asked to bring a signed copy of the consent form to their scheduled interview. For participants who were not located in the Northern, Southern or Central regions of the Okanagan Valley, or for those who wished to interview via telephone, scanned copies of signed consent forms were sent back to the researcher via e-mail prior to the start of the interview. All signed consent forms are stored securely in a locked cabinet in the Health, Ethics and Diversity Lab at UBC’s Okanagan campus.

\subsubsection{Data Collection}

As the goal of this research study was to generate rich, thematic accounts of the moral uncertainty nursing students experienced during GHPs, semi-structured interviews served as the primary mode of data collection for this research study. I conducted 17 interviews with 17 participants over a period of 5 weeks (February - March 2017). Seven interviews were conducted in-person either at the Health, Ethics and Diversity Lab at UBC’s Okanagan campus or at quiet location off campus. The remaining ten interviews were conducted via telephone. In totality, 
1070 minutes of interview data were collected, with the average interview lasting 62 minutes.

\subsubsection{Semi-structured interviewing}

Semi-structured interviews (Appendix F) are conducted when an exploration of in-depth experiences, and the meanings participants attribute to these experiences, are sought (Adams, 2010). Often conducted on a topic of interest that seeks to identify the "variability rather than the commonality of responses” (Adams, 2010, p. 18), semi-structured interviews allow participants the freedom to explore concepts that are of interest to the researcher through a reflection on their own experiences (Adams, 2010). It is important to acknowledge, however, that responses elicited through semi-structured interviews hold an element of co-production from both the interviewer and the interviewee (Kvale, 2008). While this co-production of knowledge often allows for the generation of high-quality data, researchers must try to remain "judgmentally neutral facilitators" (Thorne, 2008, p. 129) throughout the interview process (Thorne, 2008). Practical strategies for remaining neutral during the interview process include asking open-ended questions, refraining from displaying affirming or disaffirming gestures, and minimizing the number of personal anecdotes told. As Thorne eloquently states, “it requires a substantial dose of humility and reflexivity to ensure that the dominant aspects of your own personality and passion don't steer the interactions in a predictable direction” (2008, p. 129).

\subsubsection{Creation of the semi-structured interview guide}

Due to the sensitive nature of the topic explored in this study, specific attention was given to the creation of the semi-structured interview guide. In particular, attention was paid to how I approached topics encompassing the more challenging aspects of the interviewee's international practicum, and in particular, their experiences, if any, which may have been indicative of experiences of moral uncertainty. As discussed in an earlier section of this document, moral 
uncertainty occurs when "a nurse feels indecision or a lack of clarity or is unable to even know what the moral problem is, while at the same time feeling uneasy or uncomfortable” (CNA, 2008, p. 6). Thus, in an effort to explore participants' experiences of moral uncertainty, more broadly, ethical language was deliberately avoided for fear of confusing or restricting the breadth of topics that the interviewee felt comfortable discussing over the course of our conversation.

Furthermore, upon consultation with my supervisory committee, it was decided that I should gently ease into the conversation by asking broader, more approachable questions, such as “can you tell me a bit about how you came to go on a global health practicum?” and “can you tell me a little bit about this experience - what were some of the highlights for you?” When it came time to broaching the topic of the more challenging or uncomfortable aspects of the GHP, I made sure to incorporate language that did not presume that the participant experienced any morally and/or ethically challenging moments during their GHP. For instance, instead of asking “what were some of the more challenging aspects of your GHP?”, I asked “were there any times when you were uncomfortable during your global health practicum?”

\subsubsection{Interview process}

Semi-structured interviews were conducted in a location and time that was best suited to the participant. For participants located in the Central, Northern, or Southern regions of the Okanagan Valley, interviews were conducted in a private and comfortable location, either at a quiet space off campus, or in a private meeting room in the Health, Ethics and Diversity Lab at UBC's Okanagan campus. For participants who were located outside of the Central, Northern, or Southern regions of the Okanagan Valley, interviews were conducted via telephone. It was anticipated that interviews would take between 30 minutes and an hour of the participants' time. However, as I previously stated, participant interviews lasted, on average, 62 minutes. 
Prior to commencing the interview, I thanked the participant for coming, answered any remaining questions that they had, acquired signed consent forms, and ensured the participant of their confidentiality. On-going negotiation of informed consent (Thorne, 2008) occurred, and participants were made aware that they could stop the interview, pass on a question, or leave the interview at any time. Upon obtaining consent from participants, all interviews were audiorecorded. I re-assured participants that the audio recording would be transcribed verbatim, with any identifying information coded with a number to preserve - to the best of our ability - the confidentiality of participants.

Interviews began with the completion of a participant survey, which consisted of acquiring basic demographic information, as well as past and present global health experience (Appendix E). Tape recorders were subsequently turned on, and I used a pre-determined semistructured interview guide (Appendix F) to assist in asking open-ended, thought provoking questions. As I briefly discussed in an earlier section, interview questions were intentionally designed to avoid the use of ethical language such as 'ethics', 'ethical', 'moral', and 'morality'. This was done to ensure that participants felt at ease, as well as to avoid any misunderstandings with the use of ethical language. For many participants, ethical language can be confusing or is solely conflated with biomedical ethical issues. In this study, I was interested in the day to day moral tensions that arise out of nursing practice during GHPs, but which may not necessarily be interpreted as moral challenges by participants.

Verbal probing, the interviewing technique of 'probing' further into the response of the participant (Willis, 1999), was utilized as needed to gain more detailed insight into "specific information relevant to the question, or to the specific answer given” (Willis, 1999, p. 5) by the participant. Moreover, upon completion of the interview, field notes were taken to summarize the 
content of the interview, as well as to capture the physical and contextual elements of the interview that may not have been identifiable through an audio-recorded transcript (Morse and Field, 1995). Characteristics, such as the emotional climate of the interview, the non-verbal cues expressed by the participant, and the initial impressions left by the encounter were all reported on (Morse and Field, 1995). This process of contextualization helped to maintain trustworthiness and rigor (concepts which I will explore in greater detail in another section), as I documented emergent and nascent ideas, explained my rationale for following certain 'leads', and reflected on questions that I did not pose during the interview. Field notes were not, however, coded as part of the formal data analysis process. A sample format for recording field notes, adapted by the template created by Morse and Field (1995), can be found in Appendix G.

The interview process was brought to a close by encouraging participants to voice any outstanding questions or concerns that they may have had, and by asking the participant if I could contact him or her for a second interview should any questions or the need for elaboration arise (Morse and Field, 1995). However, due to the level of articulation and reflection present in the first round of interviews, the need for second interviews did not arise.

\subsubsection{Data analysis}

Interview data was transcribed verbatim, de-identified, checked for accuracy, and uploaded into the NVivo 11 (QSR International, 2017) for data analysis. In order to familiarize myself with the interview data, I transcribed nine of the 17 interviews, while the remaining eight interviews were transcribed by a transcriptionist who had signed a contract of confidentiality.

Consistent with the analytic strategy of choice for "studies in which the purpose is to uncover commonalities and patterns across cases within a human experience” (Thorne, 2008, p. 151), constant comparative analysis of the semi-structured interview data was conducted. 
Constant comparative analysis involves the process of taking one piece of data and comparing it to all other pieces of data that hold similar or conflicting accounts (Thorne, 2000, see also: Thorne, 2008, p. 151). The researcher then poses analytic questions, such as: How are these similar? Where are the differences located? Are these accounts related? It is in this comparative process, as the researcher is challenged by new ideas that emerge with fresh sources of data (Corbin \& Strauss, 1990), that possible relationships within the phenomenon of interest emerge (Thorne, 2000).

For this study, data collection and analysis occurred concurrently. Concurrent data collection and analysis affords the researcher an opportunity to continuously engage in the process of confirming, exploring, expanding, and testing ideas that begin to form as soon you enter the research process (Thorne, 2008). Several elements of this study, such as the use constant comparative data analysis techniques, are founded on the ideals of concurrent data collection and analysis. As such, it was fitting to concurrently collect and analyze semistructured interview data.

Preliminary data analysis began once the first interview was drawn to a close. This occurred through the process of writing up field notes which, as I described in an earlier section, helped to characterize the contextual features of the interview, as well as to provide a space for exploring any initial 'hunches' that I had. A more formal approach to preliminary data analysis began once three participant interviews were completed and subsequently transcribed. The initial step of data analysis was to familiarize myself with the semi-structured interview data. I transcribed the audio-recordings verbatim, reflected on written field notes, and read and re-read the participant transcripts from beginning to end, making notations and memos in the margins 
about recurrent phrases, ideas, and emerging concepts. The following excerpt is from a memo I recorded during this preliminary data analysis phase:

This idea of "perhaps my skills are better used elsewhere" is an interesting one. I think it's so interesting because it really speaks to what I believe is a disjuncture between what the GHP is intended to do, and how it leaves some participants feeling. Here at UBC Okanagan, the GHP is - and this is verbatim - "designed to provide opportunities for students to gain insight into global health issues and cultural safety”. And if this was truly being done, wouldn't participants be encouraged to see that differences across cultures exist? To engage in a process of cultural humility? This doesn't mean that the skills students have been trained in are useless, or that they can't contribute to patient care, or that their mere presence is unhelpful; it simply means that alternative approaches need to be considered. In essence, I believe it would be a matter of reframing (Research Field Notes, 2017).

Upon familiarizing myself with the initial sources of data, I began the formal coding process. Morse and Field (1995) described coding as the process of identifying and categorizing persistent themes, phrases, and words in the data. To allow for the development of sub-codes and to maintain a manageable amount of data, initial codes were broadly conceived and few in number (Morse and Field, 1995). After open coding three transcripts, first independently and then together with my research supervisor, further negotiation and formation of a preliminary codebook occurred. This preliminary codebook - composed of four overarching codes and 10 sub-codes -, was then re-applied to the original three transcripts, as well as to three other transcripts not previously coded. The results of this initial analysis were then presented to my thesis committee to further refine the coding framework, as well as to discuss any discrepancies 
apparent in these beginning coding decisions. These consultations were used not only as an opportunity to openly discuss and reflect on emergent themes, but also as a source of investigator triangulation (Denzin, 1978), and an opportunity to receive feedback and guidance from experts within this field of inquiry. Upon incorporating the feedback received from my thesis committee, the further refined codebook was then re-applied to the original interview transcripts, as well as to all remaining and previously un-coded transcripts.

After all transcripts were coded, data contained within each code were then compared using constant comparative data analysis techniques; combining codes that shared common properties and identifying the need for sub-codes within the existing and overarching categories. It was in staying 'close' to the data, reflecting on written field notes and reflective journal entries, and upon application of prolonged and critical reflection that I constructed thematic accounts, or interpretive descriptions, of the interview data.

\subsubsection{Knowledge translation}

Findings generated through this research study have been, and will continue to be, disseminated through a number of traditional dissemination activities. These dissemination activities align with what the Canadian Institutes of Health Research (2010) defines as end-ofgrant knowledge translation (KT): "the dissemination of findings generated from research once a project is completed, depending on the extent to which there are mature findings appropriate for dissemination” (p. 4). To date, I have presented the study's findings to academic audiences at UBC Okanagan's School of Nursing, as well as to those attending the $29^{\text {th }}$ Annual Canadian Bioethics Conference (May 2018). Furthermore, I intent to publish the results of this study in the peer-reviewed journal, International Journal of Nursing Education Scholarship. 


\subsection{Trustworthiness and Rigor}

Denzin and Lincoln (2011) use the terms "trustworthiness" and "rigor” to describe how the validity, or credibility, of the evidence generated through qualitative research is established. A number of strategies were employed throughout this research study to maintain considerations of trustworthiness and rigor.

\subsubsection{Reflective journaling}

The process of reflexivity is an integral component of conducting rigorous and trustworthy qualitative research (Richards \& Morse, 2013; Thorne, 2008). Thorne (2008) defines reflexivity as the process of acknowledging and documenting “...something of what is happening to you subjectively and conceptually within the research [process]...” (p. 109). The importance of reflexivity in qualitative research is predicated on an awareness of the co-constructed nature of qualitative data (Lincoln \& Guba, 1985). As Richards and Morse (2013) argue, reflexivity is "the researchers' ability to reflect on and acknowledge their place in and impact on what is studied” (p. 74). Thus, in order to make explicit any assumptions that I held in relation to the phenomenon of interest, as well as to document emergent ideas, hunches, and decisions that I made during the research process, a reflective journal was kept throughout the entirety of this research study.

\subsubsection{Representative credibility}

Representative credibility is the act of ensuring that knowledge claims generated from qualitative research studies "are consistent with the manner in which the phenomenon under

study was sampled” (Thorne, 2008, p. 224). In this study, representative credibility was established through a number of different means. Firstly, representative credibility was established by acknowledging the limitations inherent in this study design. For instance, transferability of the study's findings must be considered cautiously as the sample selected to 
participate in this study consisted of a small number of individuals from one university in Western Canada. The goal of this research was never to generalize the results of this study to a larger context, nor was it to infer that the experiences articulated in this study are characteristic of all experiences encountered during GHPs in nursing. Rather, the goal of this study was to develop insight into the moral uncertainty experienced by nursing students during GHPs, as they were facilitated by one nursing school in Western Canada. As such, this study does not attempt to make claims that are beyond the boundaries structured by the research design. Secondly, in order to corroborate and confirm the representative credibility of the research findings, field notes and reflections obtained through the use of a reflective journal were used to triangulate the research data (Thorne, 2008).

\subsubsection{Analytic logic}

To maintain confirmability of a qualitative research study’s credibility, Thorne (2008) posits that an explicit depiction of the researcher's reasoning, insight, and decisions must be made available to those outside of the research project. A commonly used approach to maintaining transparency in the research process is the generation of a research audit trail (Morse \& Field, 1995; Thorne, 2008). In this study, field notes, analytic memos, and reflections generated from keeping a reflective journal provided the basis for representing the analytic logic employed throughout the research process.

\subsubsection{Interpretive authority}

To substantiate interpretive authority, readers must be reassured "that a researcher's interpretations are trustworthy, that they fairly illustrate or reveal some truth external to his or her own bias or experience” (Thorne, 2008, p. 225). As a nurse, and a participant in a GHP during my undergraduate education, attention was given to ensuring that my preconceived ideas, 
values, assumptions, and beliefs of the phenomenon of interest were continually acknowledged and adequately reflected upon. While it would be naïve to postulate that bias can be eliminated entirely from the research process, tactics such as keeping a reflective journal, writing memos throughout the analytic process, and dialoging with my research supervisor and thesis committee helped me to acknowledge and bracket the potentially deleterious effects of researcher bias (Thorne, 2008; Tufford \& Newman, 2010).

\subsection{Research Ethics Review and Approval}

Ethical approval for this research study was obtained through the University of British Columbia Okanagan’s Behavioural Research Ethics Board. This study was guided by core principles of research ethics, such as concern for welfare, justice, and respect for persons, as outlined in the Tri-Council Policy Statement (TCPS) 2 'CORE’ Tutorial (2014). I completed the TCPS 2 'CORE' Tutorial (2014) and incorporated these core principles throughout the entirety of the research process.

Semi-structured interviews were conducted to garner a better understanding of the moral uncertainty nursing students experienced during GHPs. While it did not occur, it was possible that during the interview process distressing emotional responses may have been elicited as a result of asking participants to reflect on their overseas nursing experience. In an effort to adequately support the wellbeing of my participants, I ensured that adequate time was factored into the interview process to allow participants the time and space to express any emotions that had the potential to been evoked over the course of our conversation.

Participants' identity and reflections were kept strictly confidential. All data related to this research study is being stored at UBC's Okanagan campus in a locked, secure location that is accessible only by myself and my research supervisor (BP). Upon completion of this research 
study, data will be stored electronically on a password protected, encrypted device for a minimum of five years. This length of time is in accordance with UBC's research ethics policies. Any hard copies of the data will be permanently destroyed using the confidential recycling service employed by the university. 


\section{Chapter 4. Findings}

\subsection{Introduction}

This chapter presents the study's findings, focusing on the thematic accounts of moral uncertainty that were constructed from nursing students' experiences of global health practicums (GHPs). In accordance with the interpretive descriptive approach undertaken, four thematic accounts of moral uncertainty typifying the data are presented. In the first theme, heightened reality in the global context, I will describe how experiencing the 'exotic' nature of working in a foreign environment was a source of considerable moral uncertainty for GHP participants. In the second theme, cultural collisions in healthcare, I will describe how experiences of moral uncertainty resulted from a disjuncture between participants’ values, beliefs, and assumptions, and the new cultural and clinical care environments within which participants found themselves. In the third theme, blurring of boundaries: Scope of practice issues, I will describe how in the midst of new clinical and cultural care environments, participants grappled with issues related to scope of practice and professional responsibility. In the last theme, connectedness, I will describe the importance of the connected and collaborative relationships that participants forged during their GHP, and discuss how experiences of moral uncertainty, and subsequent frustration, arose in their absence. A pictorial representation of the four themes and respective sub-themes described throughout this chapter can be found in Table 2. 
Table 2. Thematic accounts of moral uncertainty

\section{Theme 1: Heightened Reality in the Global Context \\ 1a. Heightened Presence: "On Display” in the Global Context \\ 1b. Confronting Issues of Power and Privilege}

Theme 2: Cultural Collisions in Healthcare

2a. A Quelled Approach to Care: Experiences of Moral Uncertainty

2a.a (In)Action

2a.b Relational practice

2a.c "It's just a different type of care": Seeking clarity amidst moral uncertainty

2a.d "My hands were tied": Experiences of moral distress

\section{Theme 3: Blurring of Boundaries: Scope of Practice Issues}

3a. Contextual Factors: Laying the Foundation for Moral Uncertainty

3a.a Rurality

3a.b Misaligned expectations

3a.c Individual characteristics

3b. Experiences of Moral Uncertainty Derived from Issues of Scope of Practice 3b.a "It's nobody, or it's you"

3b.b "I’ll just watch for my own learning": Experiences of guilt and burden

\section{Theme 4: Connectedness}

4a. Something Familiar: Working Through Experiences of Moral Uncertainty

\subsubsection{Description of the Sample}

Seventeen participants were included in this research study. Fifteen participants identified as female, and two as male. The mean age of participants was 26.5 years, with a range of 23 to 31 years of age. Furthermore, the majority of participants self-identified as either 'Canadian', ‘Caucasian’, ‘white’, or ‘European’. Sixteen participants had completed a bachelor’s degree, and one participant had completed a master’s degree. Eight participants indicated that they had travelled, volunteered, or worked in the global South prior to engaging in the GHP. Seven of the 17 participants indicated that they had gone on to travel, volunteer, or work in the global South upon completion of their GHP experience. Three participants indicated that they had been in the global South both prior to, and upon completion of, their GHP. 
Participants represented five of the six years (2011-2016) from which the sample of prospective participants were recruited. No participants from 2015 expressed an interest in participating in this research study. Presently, and over the course of the time frame sampled, UBC Okanagan’s School of Nursing consistently facilitated GHPs in two African nations, one in West Africa (Ghana) and another in sub-Saharan Africa (Zambia). In the context of this research, nine of the participants chose to go to Zambia, Africa for their GHP, whereas eight participants went to Ghana, Africa.

All participants were recruited through recruitment bulletins distributed via e-mail by UBC Okanagan's School of Nursing. Recruitment e-mails were sent to eligible participants on February $3^{\text {rd }}$, 2017. Due to a rapid response rate, the recruitment e-mail was sent to prospective participants on only one occasion. Ten interviews were conducted via telephone, while seven interviews were conducted in person either off campus or in the Health, Ethics and Diversity Lab at UBC’s Okanagan campus. The average length of interviews was 62 minutes, with a range from 42 minutes to 88 minutes. In totality, 1070 minutes of interview data were collected and subsequently analyzed.

\subsection{Heightened Reality in the Global Context}

From the moment that participants landed in either Ghana or Zambia, it was as if they had entered a heightened reality. A reality that was characterized by unfamiliar sights and smells, new languages and cultural practices, disorienting healthcare settings, and previously unseen geographical features, such as lush jungles and vast savannas. In this new, and oftentimes, overwhelming, environment, participants found themselves at the center of attention, garnering the curiosity of the local community members wherever they went. Ultimately, however, it was 
in experiencing this heightened reality, that the context, or breeding ground, for much of the moral uncertainty that participants experienced during their GHP was born.

\subsubsection{Heightened Presence: “On display" in the global context}

At first, participants recalled the attention that came from being a visitor in the local communities as endearing and exciting. Participants fondly recounted stories of the local children waiting at the door, willing participants to let them accompany the group on the walk down the road. Other times, participants spoke of the bafflement, albeit excitement, when the local night club filled up within ten minutes of the Canadian nursing students being there and the DJ proceeded to play “all the white music they had, including Happy Birthday and a Christmas song”. Other times, it was the familiar sound of 'mukua, mukua!' ['white person, white person!'] being called out whenever the group of participants walked past. However, after a while, the relentless feeling of being "on display” became exhausting, and the novelty of being a 'mukua' [Zambia] or an 'obruni' [Ghana] wore off.

For some, the discomfort that came with a being "on display" arose from the isolating and objectifying feeling of being exotified. One participant described it as a feeling of exposure, whereby “people were always looking at you, and you know, they'd be craning their heads to watch you. And people...would whisper to each other”. Another participant felt discouraged when it became obvious that a number of community members only came to the health clinic to see the Canadian nursing students who were helping to run the clinic that day. A similar experience was echoed by another participant, who, as opposed to feeling defeated, regarded the situation with optimism and was hopeful that their presence resulted in the registration of some new community members to the clinic. 
While instances of discomfort, such as those described above, were not uncommon, moral uncertainty, whereby participants experienced indecision and a lack of clarity as a result of their heightened presence, occurred less frequently. In times where moral uncertainty did occur, participants grappled with questions related to professionalism and professional identity; fueling anxieties that were rooted in a fear of misrepresenting Canadian nurses as a whole. For one participant, this was exemplified when the opportunity to go out to the local club was raised:

Now that I can think about it, it was fun. I think in the moment I was so anxious about giving off a good representation of what Canadian nurses were like... and we had been working with a mission in the town, as well, so I remember being like, oh my goodness, if the mission finds out that we're dancing and drinking [laughs]. Like, what would that look like? And I remember...I just didn’t want to give a negative representation or be known as the Canadian girls who partied.

While the moral uncertainty that this participant experienced was lessened through conversations with the mission, and through the respectfulness of the participants themselves, an important reflection on the need to be culturally sensitive to the environment that you are in, remained.

\subsubsection{Confronting issues of power and privilege}

For the majority of participants, the discomfort that was associated with a heightened presence bred feeling of isolation, objectification, and at times, frustration. Interestingly, however, when participants transitioned beyond more surface level reflections of what it means to be hyper-visible, they found themselves being confronted by, and grappling with, sophisticated issues of power and privilege.

For a number of participants, privilege, and the often unwanted and unearned advantages that it affords, was an abstract theory that had previously never been called into question. It was 
in experiencing, first hand, the advantages of privilege during their GHP that participants began to grapple with the consequences of a heightened position of power. For some, it was being looked to first for medical expertise on issues that were beyond the scope and level of education of a nursing student who had been trained in Canada. For others, it was evident when they gained entrance into high level hospital executive meetings, which rarely, if ever, would include local staff from a similar level of education and experience. For one participant, acknowledging the benefits of privilege came from witnessing the profound suffering of a man dying from a disease that would be swiftly treated, and cured, in Canada:

I kept dwelling on it thinking like, the only reason why this man is dying is because of where he was born. And that was, that was - oh, that sense of white privilege was just - it made me feel sick to my stomach knowing that if I got unwell, I would probably get better. For others, however, the entirety of the GHP experience was a stark reminder of the privilege they held. The daily cues that prompted one participant to question just how much privilege the group of GHP students occupied on a daily basis, was a source of insecurity: "I was so conscious of the fact that we were this huge group of white people who would just go out and spend money and buy things on weekends”. For another participant, a heightened awareness of privilege left them weary with feelings of guilt and questioning how the group should be harnessing and utilizing their privilege, for the better. Was it in the form of purchasing medications and treatments for those who could not afford to receive the care that they needed? Was it in advocating for treatment options that otherwise might not be made available to patients? Was sustainability even an option with such actions?

For other participants, however, acknowledging issues of power and privilege, and subsequently experiencing the moral uncertainty that ensued, was an isolating, and at times, 
frustrating, experience. A number of participants struggled with how insular, and Western, the GHP seemed, and how such a narrow-minded approach to the experience failed to contribute to the development of a critical social consciousness in GHP participants:

...we went to the slums when we were in *Capital City, and some of the girls were like, oh my gosh, this is unbelievable, I can’t believe it! And I was like, where were you? What do you mean you can't believe it?

For this participant, it was as if some of the GHP participants failed to acknowledge that the communities they stayed in, and the poverty that they saw, weren't just liminal states that people came to witness - they were places where people spent their whole lives.

Similar sentiments were shared by another participant who grappled with the connection between privilege and voyeurism when recounting an experience of walking through one of the local communities:

...the instructor was trying to say, it's like someone walking down your street, and I was like, it feels different than that because we are walking down their street to be like, look how sad it is here. That's not an appropriate thing to do to someone else's home, but then at the same time, is it worth it, if all of the people that do international practicums then have a widened worldview?

For this individual, questions remained as to how best to approach the raising of participants’ critical social consciousness during GHPs while also preventing such experiences from venturing into voyeuristic territories.

\subsection{Cultural Collisions in Healthcare}

Given the nature of a GHP, it is perhaps unsurprising, and indeed, expected, that participants would experience times when the culture embodied by the host communities, 
collided with the cultural values, ideas, and customs that were embedded in the social identities of the GHP participants. For the majority of participants, exploring the local culture and embracing the values, beliefs, and social practices upon which these cultures were formed, was an incredibly rewarding and eye-opening experience. Participants spoke at length about the deep level of respect they held for the local communities and for the collective spirit of compassion, strength, perseverance, and community-mindedness that they embodied. However, while immersion in the local culture provided participants with important opportunities for critical reflection and personal growth, the collision between healthcare cultures proved to be more challenging for them to digest.

\subsubsection{A quelled approach to care: Experiences of moral uncertainty}

For many, moral uncertainty was born out of witnessing or engaging in healthcare practices and contexts that were so different than one's own. Most often, participants grappled with a perceived difference in the pace and urgency of care that informed the culture of the larger, more urban, healthcare settings. For a number of participants, this diminished sense of urgency was a source of frustration and discomfort, prompting participants to question the local healthcare providers' approach to priority setting and responsiveness to patient needs. This is turn, fueled perceptions of a less innovative, less resourceful, less urgent, and less caring healthcare system.

\subsubsection{1 (In)Action}

For one participant, frustration and uncertainty arose when the local healthcare providers did not break from their embedded routine of dusting and cleaning to respond to patients who were exhibiting signs of distress and anguish. For another, frustration was born out a failure to act on what the participant perceived to be an appropriate and achievable course of treatment: 
“....if you say this patient needs an IV or this patient need pain medication, it would be like, 'oh, well, we will ask the doctor tomorrow morning', and it's like 10 o'clock in the morning, right?" Similar sentiments were expressed by two other participants who found themselves grappling with the consequences of alternative approaches to patient care. In one instance, a participant recounted the distress that accompanied an unanswered call for help in resuscitating a young patient who went into cardiac arrest:

His breathing changed and then actually he died and the parent was looking at me and I had no idea what to do. I had never been involved in a code before...I put him flat, I started calling for help. Everyone looked at me like I was crazy. And then my partner from Canada, another nursing student that I was with, she came over and she started calling for help too because we thought we should try and save this person. Nobody came and then the patient was dead. And then I just remember being so upset. Nobody even walked over. Everyone looked at me and was like, what is she doing? In another instance, a participant was struck by the lack of urgency and action taken by the local healthcare providers when the GHP participant and her student colleague came upon three infants struggling through periods of breathlessness: "I think we were a bit surprised in that we were noticing that the infants were having these apneic spells and the nurses didn’t feel the need to take action”. Interestingly, however, after the Canadian contingent (comprised of two nursing students and their instructors) encouraged the local staff to participate in the resuscitation efforts of the apneic infants, the same participant went on discuss the moral uncertainty, and indeed, distress, that arose when one of the three resuscitation attempts was unnecessarily prolonged, and ultimately unsuccessful: 
She [the instructor] got the physician to go do CPR on this infant. Unfortunately, kind of over about say - they performed CPR for a couple of hours, at which point is usually quite long. And so, they were sort of - the *Country 1 nurses were continuing with CPR as the physician ordered, and it kind of came to a point where...I really felt that it was - everyone kind of knew it was kind of this big elephant in the room. You know, they're not going to make it, unfortunately. They were born quite prematurely...their chance of living was very low at that point...So eventually, they [the instructors] stepped in and spoke to the physician about saying, 'okay, you know what, I think we need to call this. Our goals of care have changed, and you know, I think we just need to call this'.

For a number of participants, this less action-oriented approach to care was amplified by preconceived ideas and expectations of what the culture of care would look like in the global South. For example, instead of a climate of resourcefulness, whereby staff would be "using everything that they had" to care for their patients, one participant was struck by the host healthcare providers' more subdued, less action-oriented, approach to care:

...it's kind of having the initiative to see...the need for change, or the need for improvement...having a desire for, 'yeah, okay, you know what, we can make things better'...I honestly felt that that was kind of lacking sometimes. They didn’t have that same drive to really change and look for ways to improve things.

Similar sentiments were expressed by participants who witnessed rooms full of well-functioning equipment sitting untouched and collecting dust, or upon witnessing the use of these donated supplies in ways which had the potential to evoke patient harm. While participants were quick to acknowledge the complexity and difficulty that comes with providing aid to foreign countries, 
the observations and experiences articulated above fueled participants' perceptions of a less innovative, less resourceful, and less urgent healthcare culture.

For two participants, however, discomfort was derived not from a perceived lack of urgency, or a failure to take action, but rather, from actions, at the hands of GHP participants, that were, or had the potential to be, more harmful than they were beneficial. In one instance, a participant remarked on how uncomfortable they felt when fellow GHP participants expressed frustration and unease at the lack of interventions undertaken to prolong the life of dying child. Contrary to the appeals for greater interventions from the other participants, this individual supported the local healthcare providers' approach to care, and encouraged her colleagues to reflect on the sustainability of their calls for action:

...this little girl, she had encephalopathy from malaria, so her quality of life was going to be so bad anyways and I know some of our nursing students were saying, 'oh! What can we do to help her?' But I saw it as like...I don't know if it’s best to help her other than relationally - I don’t know - because there’s not as many resources and even here [in Canada] it's sometimes like when you have a patient who has had all the life saving measures but then is non-verbal and in a wheelchair...

For another participant, questioning the helpfulness of one’s actions occurred many years after the GHP experience, upon completion of additional training in global health that included, amongst other things, content on the ethics of providing care in resource constrained contexts. For this individual, reflecting on an experience of caring for a malnourished infant served as a catalyst for examining the reasons behind, and consequences of, encouraging the provision of intervention-focused care in a healthcare environment where this type of care could not be sustained or supported. The participant remarked, “I think it was that realization of it’s just a 
different type of care...if that baby is going to survive, it will survive because it is a strong baby. Not because of the care we [as nursing students] provide”.

\subsubsection{Relational practice}

Not all participants, however, attributed the perceived difference in healthcare cultures to specific instances of inaction or lack of urgency. For some, the difference stemmed from what

participants interpreted as a less relational, more removed, approach to patient care. For example, a number of participants grappled with the discomfort that arose out of clinical environments where patients and their families were expected to display a level of stoicism that was unfamiliar to the GHP participants. Oftentimes, participants were confronted with these expectations on units where life and death occurred at a frequency that was unfamiliar to them, such as in the Neonatal Intensive Care Unit (NICU), or in the labor and delivery suites. And while participants did not expect that the approach to care in such environments would mimic their previous experiences of life and death in Canada, the majority of participants expressed discomfort upon experiencing an approach to care that was so vastly different:

There was a lot of awkwardness...mothers aren’t allowed to make noise when they're laboring and the ones who did, the nurses would like slap the side of their legs and stuff like that....and at first everyone was kind of like 'ah'...do we say something? And then day after day you realize it's like the norm.

Other times, however, participants’ perceptions of a quelled approach to patient care were exemplified by a perceived failure - on the part of the host healthcare providers - to relate compassionately to the lived experience of a patient and/or their family. For one participant, this was highlighted when local healthcare providers approached a sensitive situation with what was perceived as a diminished sense of professionalism and compassion, failing to provide a safe 
space for a young mother and her gravely ill infant. For another, the care of a miscarried infant and the treatment of her mother was a source of significant distress:

...they would put the deceased babies in a cardboard box and put it on the floor next to the garbage can. And I wasn't really morally sure how to accept that because I think of are you just putting that baby out to the trash? Like the mother doesn't get to see the baby.

\subsubsection{3 "It's just a different type of care": Seeking clarity amidst moral uncertainty}

Moral uncertainty that arose from the collision of healthcare cultures, and in particular, experiencing a quelled approach to care, was a source of considerable distress for GHP participants. In an attempt to gain a level of clarity in the midst of such disjuncture, a number of participants sought to attribute meaning, or make sense of the discomfort that they experienced. For the majority of participants, this was exemplified through thought processes and statements which - from the perspective of the participants - helped to explain why such differences in approaches to care existed.

A number of participants attributed differences in the pace and urgency of care to a culture where death, and the process of dying, had become normalized as a result of increased mortality and morbidity rates. In the eyes of these participants, a lack of urgency was simply a by-product of a system that had become accustomed to death. As one participant stated:

...it's not about judging or getting frustrated about things. It was, okay, well, this is how they do things here and that's just the way it is. It’s not that life doesn't have as much meaning, I think death just happens a lot more there than it does here. So they are a little bit more jaded towards it. 
Two participants, however, struggled to attribute the differences in approaches to care, and in particular, the approach to implementing life sustaining measures, as solely related to a higher incidence of morbidity and mortality. While these participants acknowledged that increased rates of death and dying did contribute to a culture where death was normalized, and a lack of urgency prevailed, these participants made meaning of the moral uncertainty they experienced by attributing it to a lack of resources and a difference in the meaning and value that life held in the local cultures. As one of the two participants remarked:

...if you think about the same situation you know, back home in Canada, it would be completely different, and you'd have, you know, everything at your fingertips. And I get the feeling that life would be more valued if it was here. Because there's more money that would be put into that person - baby, and stuff like that.

For another participant, however, perceived differences in the pace and urgency of care arose not from more tangible disparities, such as increased morbidity and mortality rates, or a lack of resources, but rather from the value local healthcare providers, and indeed, the larger community, placed on divine intervention. For this participant, local healthcare providers' faith in "God's will” helped to explain why steps to resuscitating infants were not as actively pursued as they would be in Canada.

Other participants sought to make sense of the discomfort that resulted from a collision of healthcare cultures through attempts at understanding what it would be like to practice solely in a healthcare environment that was perpetually resource constrained. For a number of participants, differences in the pace and urgency of care were attributed to expressions of resignation, which in turn, gave rise to attitudes of complacency. In the minds of these participants, local healthcare providers felt resigned to the system they operated in, and thus, failed to display a will to 
innovate and improve the conditions within which they operated. It was through this lens of understanding that one participant remarked: “...I feel like the nurses are there, but like it has gotten to this point where they just don't do anything anymore, so they dust. They keep the ward clean, but they just let everything else run its natural course”.

For others still, experiencing the moral uncertainty that arose from a collision of healthcare cultures was a catalyst for reflecting on one's future contributions to the field of global health. In such instances, the meanings attributed to the cultural divide centered on the perception that the chasm it created was simply too large to be bridged. Oftentimes, such reflections would occur when participants were overwhelmed by the seemingly insurmountable differences between the two healthcare systems and saw little opportunity for meaningful engagement. One participant indicated that they would only engage in future global health work if "the culture of the organization aligns with the culture that I'm used to”. For others, the profound difference in culture norms restricted one's ability to engage efficiently in nursing practice, prompting feelings of being resigned to practicing in Canada where a fuller understanding of cultural norms and social practices was known. Lastly, for one participant, the differences in healthcare cultures, and having the ability to withstand and thrive in these differences, was a humbling and disheartening experience:

I remember coming home feeling like I didn’t have what it took to be tough enough to be a nurse in a developing country. And it was really - that was really devastating because that was like my whole life goal, was to become a nurse and travel overseas.

\subsubsection{4 "My hands were tied": Experiences of moral distress}

Not all participants, however, were able to reconcile the moral uncertainty that arose out of the collision of healthcare cultures through thought processes which sought to explain why such 
differences in healthcare cultures existed. For these individuals, a position of moral distress was adopted. By moral distress, I mean the stress that arises "when one knows the right thing to do, but institutional constraints make it nearly impossible to pursue the right course of action” (Jameton, 1984, p. 6). In other words, moral distress occurs when individuals are "unable to translate moral choices into moral action” (Ericksen, Rodney, \& Starzomski, 1995, p. 32)

For the majority of participants, moral distress was cultivated out of instances where participants witnessed or engaged in practices that fostered tension between respecting the values and beliefs of the local healthcare system and upholding the moral judgment and subsequent value systems of participants. Oftentimes, these experiences were characterized by accounts of discomfort, followed by phrases such as “it's not my place to make that decision”, or "my hands were tied”. For instance, when a participant expressed uncertainty, and indeed, discomfort, upon witnessing the care of a miscarried infant and the perceived maltreatment of her mother, questions relating to how best to process feelings of moral distress arose:

At first, I was like, I don’t know what to do because it's obviously the way they handle that, and at the same time it was like how horrible a way to handle that. And I had to keep being like you're not in Canada, you're here, kind of thing. But does that make it morally okay? You are in another country and in a different cultural setting, but does it still make it okay?

For another participant, much of the GHP experience exemplified feelings of moral distress, contributing to rising feelings of frustration in being unable, or constrained, in vocalizing and acting upon one's moral judgment. In the end, the uncertainty left the participant questioning what the objective of the GHP was: 
...it's challenging to be like, 'well, we should be doing this', like that seems so unethical to me...it's not my place to say anything. And it's not like me being here for one day is going to change anything. So, I found that really challenging, just kind of standing there for the day thinking, 'you should be doing something about this', but really like - because it seems like from the whole experience you're not going to make any real change, so, I don't know if the goal of the program was just to show you a bunch of stuff and then leave?

\subsection{Blurring of Boundaries: Scope of Practice Issues}

In the midst of experiencing a heightened reality, and in the disjuncture that resulted from cultural collisions in healthcare, participants found themselves challenged and intrigued by issues related to scope of practice and professional responsibility.

\subsubsection{Contextual factors: Laying the foundation for moral uncertainty}

A number of contextual variables, such as rurality, lack of supervision, misaligned expectations, and individual participant characteristics, influenced experiences of moral uncertainty as they related to issues of scope of practice and professional responsibility.

\subsubsection{Rurality}

Experiencing the exotic nature of a rural, 'outreach' opportunity was a source of great satisfaction for many participants. In particular, participants remarked on the importance of rural practicums for gaining insight into the culture of the host nations, for feeling as though they were able to give back to the local communities, and for exemplifying what they had envisioned as a true 'global health’ experience. During these rural, ‘outreach’ opportunities, participants found themselves in geographical locations which defied previous experiences of working in rural healthcare settings: 
...to give some context, we land in *City 1 , which is the capitol, and it's huge, it's you know, a big metropolitan area. And we had travelled 12 hours by bus to *City 2, which is the capitol of *Geographical Location 1 - and now we're getting very, very, very rural [laughs]. And then we were 40 minutes, or an hour outside of there, so now we're in the villages. And so, the outpost that I was working in for a week, was an hour past that...so, a really, really small, rural area.

While participants found themselves challenged and intrigued by the rural nature of these clinical rotations, for many, the variables that accompanied, and indeed, contributed, to making these rural settings so unique, created a foundation for challenges related to practicing beyond one's scope of practice.

Oftentimes, participants found themselves in clinical settings that lacked appropriate supervision, or environments which placed a large portion of the responsibility of care in the hands of participants directly:

...it was just totally different - yeah, so during those three days, there was - there was no physician. It was just us [two Canadian nursing students and a local nurse], and a couple of translators, and you know, this 'village without a doctor” book. But you know, we would assess people, and gather information, and read our 'village without a doctor' book and try to put it together [laughs]...I'm not saying it was right or not, but you know, we were able to prescribe and diagnose - well tentatively diagnose [laughs].

Other times, participants found themselves in positions of responsibility because they were either deemed to be, or perceived to be, the most competent healthcare provider available. Participants attributed this dearth of supervision in part to larger health and human resource issues, but also to what they described as a lack of clarity and discussion around the roles and responsibilities of the 
student participants. For instance, one participant experienced a profound lack of clarity during the rural, 'outreach' portion of the GHP:

[I]n the hospital I think there was definitely more of a guideline... of what you were going to be doing for the day... whereas in those outreach areas, in those smaller clinics, it was a little bit different...we went in and said, 'Hey, we're here. What can we do?' And they didn’t really know what we could do. Potentially, they didn't really care to have us there; probably a burden on them, really. And yeah, it was kind of like...they don't want to be rude, but you know, they're kind of like 'well, you can do this, or you can do that'. And it ended up being a lot of kind of sitting around and waiting for other people to make decisions about what you were going to do for the day. And they would be like, 'well, I didn’t know you guys were coming, so I’ll talk to this person'.

\subsubsection{Misaligned expectations}

Participants often spoke of the uncertainty around what they should, or could, be doing in clinical practice. Most often, participants recounted that ambiguity lay not in the participant's own understanding of scope of practice, but in what participants perceived as a lack of clarity on the part of the local healthcare providers. A number of participants described experiences whereby host healthcare providers expected participants to be practicing at a level that was beyond the education and skill-set of a Registered Nurse, let alone that of a nursing student. Thus, when opportunities arose, host healthcare providers offered, or “...would kind of push...” participants into participating in care that was outside of their scope of practice. Other participants felt that the host partners didn't have a clear idea of what the actual practicum entailed, and subsequently, would be confused when participants indicated that they were simply there to observe and ask questions. For instance, one participant recounted an experience 
whereby the host healthcare provider questioned why the participant was in the labor and delivery ward if it were not to help with the deliveries firsthand.

Expectations, however, were not always misaligned and rooted in the host partners' perceived lack of understanding. For a number of participants, the opportunity for skill building or engaging in a practice they had not previously engaged with in Canada, was an expectation that was engrained from the outset of the GHP:

That was kind of a thing that was pumped up, like before we left. Like, 'you might get to deliver a baby!' And I was like, oh my gosh, that would be amazing. And so, sure enough, in maternity, you know, after I'd watched a couple, the midwife goes, 'okay, it's your turn to catch the baby!' And I remember being like 'What!? What!? But then that like - that eager, curious nursing side of me was like, yeah, I want to do this!

Thus, as was evident in the previous anecdote, when opportunities to engage in exciting and long anticipated practices arose, oftentimes, participants eagerly joined in on the experience. However, as the same participant later remarked, “...I think that’s where maybe that assumption of our skills and education, kind of combined with my eagerness to try new things, kind of just converged to make a potentially dangerous and definitely not ethical situation”.

\subsubsection{Individual characteristics}

While opportunities to partake in practices beyond one's scope of practice were augmented by contextual variables, such as rurality, lack of supervision, and misaligned expectations, participants also remarked on personal attributes that had the potential to influence one's willingness, or unwillingness, to engage in practices outside of one's scope of practice. A number of participants reflected on how young they were at the time of their GHP, and how immaturity and a lack of experience with decision-making contributed to instances of practicing 
beyond one's scope of practice. Others attributed such decisions to variable understandings and interpretations of professional responsibility and personal accountability. For instance, a number of participants held the firm belief that their scope of practice as a nursing student remained consistent regardless of the whether they were in Canada or in Africa. However, as opportunities to practice outside of one's scope of practice were present, the decision to engage, or not, was largely left up to the individual:

....it was really up to me, so if I were the type of student that wanted to just be very independent and not ask the doctor questions, or confirm things, or question things, then there is a possibility that people may not have received the right healthcare that they should have.

Perhaps unsurprisingly, participants who had previous experience in having to draw boundaries as they relate to scope of practice were oftentimes more comfortable refraining from engaging in skills that were beyond their level of education and expertise.

\subsubsection{Experiences of moral uncertainty derived from issues of scope of practice}

Experiences of moral uncertainty as they relate to issues of scope of practice were evident throughout all 17 participant interviews. Indeed, when participants were asked to reflect on some of the more troubling aspects of their GHP experience, issues related to scope of practice were oftentimes the first thing participants recounted. Interestingly, participants were able to label such experiences of uncertainty more readily as either a moral and/or an ethical problem, as opposed to merely the 'discomfort' participants experienced throughout the other themes. However, despite a readiness to locate experiences of uncertainty as either an ethical and/or a moral issue, the depth of moral uncertainty that participants experienced varied from one to the 
next. Nevertheless, for most, the excitement that came with practicing outside of one's scope of practice was tempered by questions related to professional obligation and a duty to provide care.

\subsubsection{1 “It's nobody, or it's you”}

For a number of participants, moral uncertainty arose out of instances whereby participants engaged in, and were distressed by, an expanded scope of practice, in part, because of a dearth of support and supervision. Participants acknowledged that prior to embarking on the GHP they were told to prepare for clinical encounters that would challenge their ability to stay within a predefined, and legally defined, scope of practice. Participants recalled how they would react to such situations by asking oneself, “am I trained to do this skill?”, “am I competent in this practice”, “do I feel comfortable in performing this skill?”, “would I be engaging in this practice at home?” Indeed, it was very clear to the majority of participants, that should they feel uncomfortable engaging in a practice at any point, they could acknowledge that discomfort and take a step back. However, while such lines of questioning and self-awareness were clear in the abstract, for a number of participants, immersion in a foreign healthcare environment caused a blurring of those boundaries.

For many, indecision, or a lack of clarity, around issues of scope of practice were influenced by feelings of professional obligation. Instances, whereby participants engaged in practices beyond their level of comfort, education, and ability because of a sense of duty to provide care in the environment - and to the individuals - within which they found themselves. For a number of participants, taking a step back and vocalizing discomfort in practicing beyond one's scope of practice was simply not something these participants found that they had the luxury of doing. For example, two participants described instances during their rural, 'outreach' practicum whereby they found themselves in positions of care that would not be filled should 
they choose to leave, or decide not to participate in, the care practices they were providing. For these participants, the discomfort that came from saying, “no, I'm here to observe”, outweighed the discomfort that came from engaging in practices that were outside of one's scope of practice.

For one participant, engaging in the provision of healthcare services beyond one’s scope of practice and level of comfortability stemmed from a place of being the most educated, and thus, presumably, the most competent healthcare provider available. While this individual felt a sense of obligation to provide care to those who came in search of assistance, she experienced considerable distress knowing that "patients were coming through expecting a health professional, and they weren't getting it”. In the end, the participant was left questioning what the health outcomes of these patients would have been had they not sought out treatment from the student clinicians. Would they have been better? Would they have been worse? With a limited amount of time in each setting and knowing that “...I couldn’t do anything for these people, because I didn’t know enough...”, little clarity would be provided to this participant: “And you know, then we left. And she was still there, and you just hope that it worked out for her, you know?”

In the experiences of moral uncertainty addressed above, the phrase 'it's nobody, or it's you', embodied instances whereby participants engaged in, and were distressed by, an expanded scope of practice, in part, because of a dearth of support and supervision. However, for another participant, moral uncertainty was derived from an experience that encapsulated, amongst other things, a different definition of 'nobody'. In this instance, 'nobody' referred not to a dearth of support and/or supervision in the physical sense of the word, but rather, to a lack of confidence in the capacity and clinical competence of the local healthcare providers who were present. 
For this participant, when an eagerness to engage in a practice that would seldom arise in Canada, was met with feelings of under preparedness, the result was an expression of moral uncertainty that fueled a line of convoluted questions and reflections. The participant began to reflect on where her eagerness, and subsequent action, in birthing a child - "without feeling like that's something - a skill I should necessarily be performing" - came from:

I totally could have said no, and in the situation, they would have been - the nurses and the staff would have been fine with that. I think because it was presented as an opportunity that I would not have if I was in Canada, I was keen to take advantage of it in that sense. But at the same time, feeling underprepared or unqualified...then you feel like, well just because this is a country that doesn't have as much as much as we do...unqualified people are allowed to just come in and you know, deliver babies?

Interestingly, like a number of other participants who rationalized times of practicing beyond one's scope of practice on the basis of being the 'safest alternative' present, this individual questioned whether or not she was indeed the most qualified healthcare provider available, despite being in the presence of a number of other licensed healthcare providers.

For some participants, however, practicing outside of one's scope of practice was seen not from a lens of moral uncertainty, but from a lens of moral necessity, whereby their presence and ability to engage in practice was seen as a safer alternative to the care that was currently being provided, or, at the very least, better than no care at all. For example, while one participant felt underprepared and "panicked" when found in a situation of having to deliver a child, this participant went on to deliver three more children during her time overseas simply because a more qualified healthcare provider was unavailable at the time of delivery. Another participant felt that practicing beyond one's scope of practice was justified on the basis of having witnessed 
and lost confidence in the care that was being provided by the local healthcare practitioners: “...what I had seen in other wards and stuff, I didn’t have a lot of confidence in what they could do or would do".

However, this justification and rationalization of practicing outside of one's scope of practice was firmly discounted by a number of other participants. Oftentimes, individuals disputed the practice on the grounds of safety, and more nuanced questions on how we approach the care of underserved populations. For example, for one participant, the practice was disputed on the grounds of informed consent: “...if given the knowledge that this is a student from Canada who has never done this [skill] or learned about it before, I'm pretty sure anyone in their right mind would say, no”.

\subsubsection{2 "I'll just watch for my own learning": Experiences of guilt and burden}

For a number of other participants, moral uncertainty lay not in a perceived obligation to provide care, but in the guilt that was cultivated when participants did take a step back into the observer role. For some, guilt was derived from a failure to fully engage and participate in care practices which - in the eyes of this participant - were enmeshed in the objectives of the GHP:

So there were a number of times where I did step back and said like, no, I don’t feel comfortable. And I felt a little guilty because you know, here I am having come so far to you know, provide some sort of help to people, and you know, I'm just taking a step back and being like, no, I’ll just watch for my own learning.

For another participant, moral uncertainty resulted from conversations with the local healthcare providers who - upon learning that the participant was simply there 'to observe' - questioned what the objective of the practicum was if it wasn't to engage directly in clinical practice. Admittedly, the participant remarked that these comments were usually made in good humor, 
however, when combined with the participant's eagerness and genuine desire 'to help', it was enough to push them into providing care that exceeded both their comfort level and scope of practice.

For another participant, moral uncertainty stemmed from a fear of burdening an already burdened healthcare system. In this instance, the participant was caught between feelings of discomfort in the level of responsibility and autonomy of practice, and not wanting to repeatedly ask the sole healthcare provider - who was there to orchestrate the clinic - for advice and consultation when questions in practice arose:

...I felt bad stopping her to ask questions, when she had to see, you know, 60-70 people in four or five hours. Because that was her day...so you don’t want to trouble them too much to try and ask a bunch of questions.

Lastly, while feelings of guilt, uncertainty, and discomfort were not uncommon, for one participant, what was perceived as moral uncertainty in the moment, transitioned into moral distress after a period of deep reflection:

...I remember this one time...the baby came out and she [the mother] had a really big vaginal tear and the midwife said, 'okay, you can stitch it up'. And I remember saying, 'no, I don't know how to suture!' And she said, 'here, I'll show you'. And she showed me two stitches and told me to stitch the rest. And looking back I - I did. I stitched her up...But I look back and I go, 'I had no idea'. I had never stitched anything in my life. And never even learned about it. And here I was stitching up someone’s vagina. Like a young girl, who's going to have more babies down the road. And now knowing what I know about giving birth and perineal tears and even like childbirth in Africa, I just - I'm horrified that I 
did that. And I do wish that I had the knowledge to say no to that. And I just hope to this day that her health hasn’t been impacted further.

For this participant, reflecting on the implications of practicing beyond one's scope of practice had a lasting effect. It was as if the act of over-stepping one’s professional boundaries had instilled a sense of deep and enduring uncertainty in the participant, leaving them with a diminished level of confidence and lack of clarity in how best to proceed in a career where working overseas was still an area of interest: "I'm now so scared of doing something unhelpful, that I don't know what to do".

\subsection{Connectedness}

When participants were asked to expand on the more rewarding elements of their GHP experience, the majority of participants recounted anecdotes or experiences whereby a sense of connectedness - a sense of belonging - was evident. Oftentimes, however, such experiences of connectedness sat in juxtaposition to the experiences of moral uncertainty typified in the themes heightened reality in the global context, cultural collisions in healthcare, and blurring of boundaries: Scope of practice issues. Nonetheless, these experiences of connectedness were of paramount importance in helping participants to redress and reflect on such experiences of moral uncertainty.

Perhaps unsurprisingly, feelings of connectedness were facilitated by invitations or experiences of inclusivity; times when participants felt that their presence in the host community was welcomed, wanted, and appreciated. For a number of participants, a sense of belonging was exemplified through evenings spent laughing, joking, and sharing stories around the campfire after a busy day in the rural, 'outreach’ clinic. For others, a sense of belonging was fostered when community members invited participants to partake in community activities, such as going 
to church and playing in 'pick-up’ soccer games. For others still, a sense of belonging was cultivated through direct experiences of hospitality: invitations to attend the wedding of one of their nursing colleagues; outfitting participants in locally sewn, traditional outfits; and inviting participants to dine with them within the confines of their home.

Once a sense of belonging had been cultivated in the clinical setting, participants recounted that this feeling of connectedness served as an important conduit for collaboration between GHP participants and the local healthcare providers, helping to bridge some of the chasms created by cultural collisions in healthcare. Participants described collaborative practices as times of reciprocal knowledge development and exchange. For example, times when GHP participants were asked to accompany the ward nurses and doctors on morning rounds, or when an impromptu 'buddy system' was set up between the local nursing students and the GHP participants as a means to facilitating the delivery of culturally sensitive care:

It was a really great relationship that we had with the nursing students. They loved learning from us and they loved the tools that we brought them...or the tricks that we had learned to do...I don't think we could have gotten through our practicum without them because they helped with the translation and for us to kind of coast through to understand the way things are done...I probably couldn’t have done anything without them.

For many, collaboration with the local healthcare teams exemplified what the objective of the GHP ought to be: a conduit for gaining insight into the culture and lived-experience of the host communities; just as it did for this individual:

I remember making friends with some of the nurses and I really enjoyed...the comradery with them, and yeah, just learning what nursing was like for them. What even home life was like... what raising kids was like. And I always grew to respect them, like they worked 
so hard and by - by our Canadian standards, were understaffed, overworked, underpaid, and the burden of responsibility was huge. And yet, they took the time to teach us, and be beside us, and have fun with us on the ward.

Not all GHP participants, however, experienced the profound benefits of connected and collaborative relationships with the healthcare providers from the host communities. For three participants, being welcomed into the host communities with ease and excitement was an expectation that simply did not come to fruition. Instead of the warm welcome that participants were expecting, they were met with what was perceived as judgment, uncertainty, and an unwillingness to engage. Oftentimes, these expectations were influenced by preconceived ideas of how the host community would respond to the presence of GHP participants. As one participant remarked, "I thought that we would be very well accepted, very well loved. They would embrace us like their brothers and sisters”. When this sense of belonging was not immediately apparent, the disjuncture that ensued gave rise to feelings of uncertainty and frustration.

For most, frustration was born out of the host healthcare providers' perceived unwillingness to engage with the GHP participants. This resulted in failed attempts of collaboration, and a growing sense of uncertainty as to whether the presence of GHP participants was wanted. One participant likened attempts of collaboration to working on either side of an electrical fence, where “...the fence never goes down, there’s always electricity”. For others, despite repeated attempts at engaging with the local healthcare providers in the clinical setting, a willingness to collaborate remained absent. This resulted in diminished efforts to seek out engagement and a growing chasm between the two healthcare cultures. As one participant stated, “...I started talking to the nurses less, because it was dead road, after dead road”. 


\subsubsection{Something familiar: Working through experiences of moral uncertainty}

In times of uncertainty, participants frequently sought solace in their fellow GHP participants, looking to connect and reflect on the shared understanding of participating in a nursing practicum in the global context. For a number of participants, the ability to reflect on some of the more challenging aspects of the GHP was of paramount importance in maintaining a sense of equilibrium during what was, at times, a disorienting experience. For some, the physical presence of another GHP participant in the clinical setting was an important step in building an environment whereby critical reflection was supported and encouraged. For another, knowing that an additional GHP participant was present in the clinical setting, was source of comfort in times of uncertainty:

...going through these difficult experiences, and you don't know anyone else in the room and you look over and there's this one other person that you've travelled there with, and you sort of hold on to that connection, like - you know, something familiar, at least.

For others still, simply knowing that other participants were experiencing similar emotions was a source of reassurance, and an entry point for grappling with some of the uncertainty that participants were experiencing. Two participants emphasized the importance of continuing to connect with their fellow GHP participants upon return home from their overseas experience. For these individuals, the ability to continue the ‘debriefing' process upon return home to Canada facilitated the beginning stages of reconciling some of the uncertainty and helplessness that they had experienced during their GHP. For another, the dearth of opportunities to continue a reflexive dialogue upon return home was apparent, prompting an appeal to include postdeparture debriefing as part of the larger GHP program. 
While many participants sought out something familiar in the relationships they forged with fellow GHP participants, others recounted times when something familiar represented an avenue for a temporary escape from the unfamiliar, and at times, disorienting, environment within which participants found themselves. For example, participants spoke warmly of nights spent in their housing complex cooking dinner and watching episodes of the American television series, Glee. Other times, participants recounted the comfort that accompanied evenings spent eating chocolate chips and discussing the various experiences participants had encountered that day. For a number of participants, however, the familiarity simply served as a means to creating an environment that encouraged participants to decompress and recuperate after another long, and oftentimes, overwhelming, day of their GHP.

\subsection{Summary}

In this chapter, the study's findings were described. Semi-structured interviews were conducted with 17 graduates from UBC Okanagan’s School of Nursing who participated in a GHP between the years of 2011 and 2016, inclusive. Participants ranged in ages from 23 to 31 years of age, with eight participants indicating that they had travelled to, or worked in, the global South prior to participating in their GHP. The mean length of interviews was 62 minutes.

Experiences of moral uncertainty were evident throughout all 17 interviews. Using constant comparative data analysis techniques, four main themes typifying the data were constructed. In the first theme, heightened reality in the global context, I described how participants experienced varying levels of moral uncertainty when confronted with heightened positions of power, influence, and visibility. In the second theme, cultural collisions in healthcare, I described how experiencing an alternative approach to care, and in particular, a quelled approach to care, was a source of significant moral uncertainty, and at times, moral 
distress, for participants. In the third theme, blurring of boundaries: Scope of practice issues, I described how practicing beyond one's scope of practice contributed substantially to participants' experiences of moral uncertainty, and less frequently, moral distress. Lastly, in the fourth theme, connectedness, I described how a sense of belonging - a feeling of connectedness - was of paramount importance in helping participants to redress and reflect on experiences of moral uncertainty that occurred during and upon return home from their GHP experience. 


\section{Chapter 5. Discussion, Recommendations and Conclusions}

\subsection{Introduction}

In this chapter, I will discuss the contributions of this study to understandings of the ethical implications of global health practicums (GHPs), and more specifically, understandings of undergraduate nursing students’ experiences of moral uncertainty during GHPs. I will begin by summarizing the findings explored in Chapter Four, outlining nursing students’ experiences of moral uncertainty as it occurred during, and upon return home from, GHPs. I will then engage in a discussion around the primary contributions from this study’s findings, locating each contribution within the existing literature. I will then conclude by exploring the study's contributions to both education and practice and discuss recommendations for future research. For clarity purposes, moral uncertainty will henceforth be used to refer to all degrees of moral ambiguity, including moral distress.

\subsection{Summary of Findings}

This interpretive descriptive study explored undergraduate nursing students’ experiences of moral uncertainty during GHPs. Using constant comparative data analysis techniques, four main themes typifying the data were constructed. Experiences of moral uncertainty were evident throughout all 17 participant interviews. In the first theme, heightened reality in the global context, I described how, for many, experiences of moral uncertainty arose out of the students' heightened positions of power, influence, and visibility. In the second theme, cultural collisions in healthcare, I described how participants grappled with the moral uncertainty that resulted from experiencing alternative approaches to healthcare, and in particular, a quelled approach to care. In the third theme, blurring of boundaries: scope of practice issues, I described how practicing beyond one's scope of practice contributed substantially to participants' experiences 
of moral uncertainty. Lastly, in the fourth theme, connectedness, I described how a sense of belonging - a feeling of connectedness - was of paramount importance in helping participants to redress and reflect on experiences of moral uncertainty during, and upon return home from, their GHP experience.

\subsection{Discussion}

In this section, I will discuss the primary contributions that this study makes to the literature on undergraduate nursing students' experiences of GHPs. I will begin by presenting a discussion on the role of expectations in nurses' experiences of moral uncertainty, more broadly, followed by a discussion on the role of expectations in participants' experiences of moral uncertainty as it occurred during GHPs. I will then discuss the concept of moral uncertainty, illustrating how participants processed moral uncertainty during their GHP and conclude with a discussion on the usefulness of critical pedagogies in helping students to process feelings of moral uncertainty. Specific recommendations for education, practice, and future research will then be discussed.

As indicated above, this discussion will revolve largely around the concept of moral uncertainty. While experiences of moral distress were articulated in the anecdotes shared by participants in this study, such experiences occurred at much lower frequency than nursing students' experiences of moral uncertainty during GHPs. As such, the coming sections will feature discussions that are grounded primarily in nursing students' experiences of moral uncertainty during GHPs. However, the implications and recommendations that follow from these discussions, can be similarly applied to the occurrences of moral distress that were less frequently, albeit importantly, articulated by participants. 


\subsubsection{The role of expectations in nurses' experiences of moral uncertainty: What does the existing literature reveal?}

Findings from this study indicate that participants' experiences of moral uncertainty often resulted from a disjuncture between what participants expected to occur during their GHP, and what occurred in reality. Participants experienced an array of emotions stemming from misaligned expectations; ranging from feelings of anger and frustration, to feelings characteristic of guilt and helplessness. While a paucity of literature specific to nurses' experiences of moral uncertainty makes it difficult to embed these findings in the existing literature, a parallel body of literature detailing nurses’ experiences of moral distress, more broadly, has articulated similar emotional responses during times of moral and/or ethical discomfort (Erlen \& Frost, 1991; Pauly, 2012; Rodney \& Starzomski, 1993; Rodney \& Varcoe, 2001; Rushton, 1992; Yeo \& Ford, 1996). As such, discussions will henceforth be grounded in the existing literature on moral distress to further our understanding of the role, if any, of expectations in nurses' and nursing students' experiences of moral uncertainty and distress.

The concepts of moral uncertainty and moral distress were initially introduced to the nursing literature by American ethicist, Andrew Jameton, in 1984. In his germinal text on nursing ethics, Jameton provided nurses, and other healthcare providers, with a framework for helping them to distinguish between three categories of ethical issues: moral uncertainty, moral dilemma, and moral distress (1984). Moral uncertainty, Jameton posited, "arises when one is unsure what moral principles or values apply, or even what the moral problem is” (Jameton, 1984, p. 6), and is often manifested by nurses in feelings of discomfort and frustration (Cohen \& Erickson, 2006), as well as generalized unease (Hamric, 2000). Moral dilemmas, however, occur when two, or more, moral principles apply, "but they support mutually inconsistent courses of 
action. It seems terrible to give up either value, and yet the loss seems inescapable” (Jameton, 1984, p. 6). Finally, Jameton suggested that moral distress "arises when one knows the right thing to do, but institutional constraints make it nearly impossible to pursue the right course of action” (Jameton, 1984, p. 6). While the concept of moral distress has received notable and growing attention in both the empirical and theoretical nursing literature (Rodney, Brown, \& Liaschenko, 2004), few studies to date have explored nurses’ experiences of moral uncertainty. As Jameton (1984) initially suggested, nurses' experiences of moral distress - those feeling that arise "when nurses are unable to act according to their moral judgment” (Rodney, 2017, p. S7) - are largely influenced by institutional barriers which include, but are not limited to, legal restrictions, power differentials, and resource constraints (Hamric, 2000). For instance, in today’s healthcare climate which is often plagued by economic constraints as well as health and human resource shortages (Cohen \& Erickson, 2006), it can be “overwhelming for nurses who try to uphold the basic duties of patient advocacy and high ethical principles” (Cohen \& Erickson, 2006). Thus, for nurses, and other healthcare professionals, who are intimately involved in morally significant relationships with sick, and oftentimes, vulnerable, individuals (Bartholome, 1998, as cited in Hamric, 2000), an inability to act as an autonomous moral agent (Rodney, Brown, \& Liaschenko, 2004), is, to some effect, an inability to live up to one's own expectations, as well as to the expectations of others.

Interestingly, research indicates that nursing students - the population of interest in this study - may be more likely to experience moral uncertainty and may be at a heightened risk for being more profoundly troubled by ethical dilemmas (Cohen \& Erickson, 2006). For nursing students, and new graduate nurses, tension often arises as a result of differences between the ethical principles students are taught in school and what is experienced, or perceived, in practice 
(Cantrell, Browne \& Lupinacci, 2005). Further, nursing students’ and new graduate nurses' experiences of moral uncertainty and moral distress are often precipitated by a failure to live up to expectations put in place by healthcare institutions (Kelly, 1998), as well as one’s own expectations, or ability, "to be the kind of nurse I want to be" (Kelly, 1998, p. 1140). In the context of our findings, whereby nursing students are engaging in practices in an entirely new healthcare system and culture, it seems plausible that students would be at ever heightened risk for moral uncertainty, given the unfamiliar ground upon which they are practicing.

\subsubsection{The role of expectations in nursing students' experiences of moral uncertainty during GHPs}

As I briefly discussed, narratives which amplified participants' experiences of moral uncertainty often revealed a growing disjuncture between what participants had anticipated, or expected, to occur during their GHP, and what was experienced in reality. In the following section, I will further explore the connection between expectations and moral uncertainty, through discussions on nursing students' motivations for engaging in GHPs, as well as the role of misaligned expectations in participants' experiences of moral uncertainty.

\subsubsection{Nursing students' motivations and expectations for engaging in GHPs}

To date, a limited, albeit insightful, body of literature dedicated to exploring nursing students' expectations and motivations for engaging in overseas practicums exists (Burgess, Reimer-Kirkham, \& Astle, 2014; Gower, Duggan, Dantas, \& Boldy, 2016; Kelleher, FitzGerald, \& Hegarty, 2016; Kent-Wilkinson, Leurer, Luimes, Ferguson, \& Murray, 2015). Perhaps unsurprisingly, existing literature suggests that undergraduate nursing students are primarily motivated to participate in GHPs from positions of altruism - or a desire to help - (Burgess et al., 2014; Gower et al., 2016), from the allure of adventure and excitement (Gower et al., 2016; 
Kelleher et al., 2016), and from a sense of cultural intrigue (Burgess et al., 2014; Gower et al., 2016; Kelleher et al., 2016; Kent-Wilkinson et al., 2015). Enmeshed within these motivating factors, nursing students expected to come away from international clinical placements with an enhanced cultural awareness and ability to serve the needs of an increasingly diverse patient population (Burgess et al., 2014; Gower et al., 2016; Kelleher et al., 2016; Kent-Wilkinson et al., 2015). Oftentimes, such expectations were perceived to be met through an engagement with, and observation of, different cultural, social, and clinical care environments (Kelleher et al., 2016).

Beyond the pull of enhancing one’s cultural awareness and cultural competency, research on nursing students' motivations and expectations for participating in overseas practicums indicates that students view GHP-like opportunities as a means to facilitating both professional and personal growth. Specifically, nursing students expected to experience growth in realms such as increased confidence and autonomy (Gower et al., 2016; Kent-Wilkinson et al., 2015); enhanced language skills, communications skills, and future employability (Gower et al., 2016; Kelleher et al., 2016; Kent-Wilkinson et al., 2015); resilience and resourcefulness (Gower et al., 2016); and an enlightened perspective on the importance of social accountability, and the emerging concept of global citizenship (Burgess et al., 2014; Gower et al., 2016).

This study's findings resonate with much of the existing research on nursing students' motivations and expectations for GHPs; including, to varying degrees, literature which has identified a level of paternalism - or what can be construed as paternalistic - in nursing students' motivations for engaging in GHPs (Burgess et al., 2014). Burgess et al.'s (2014) qualitative exploration of the topic found that while nursing students were genuinely motivated to participate in GHPs from positions of altruism, "subconscious expressions of ingrained social constructs about low-income countries" (p. 79), were also apparent. These "subconscious 
expressions” (Burgess et al., 2014, p. 79) were often expressed through narratives which implied a sense of responsibility for the host populations, such as when participants spoke of providing education to the host communities, or of modeling appropriate nursing behaviour in the clinical setting (Burgess et al., 2014). Thus, as was demonstrated in this study's findings when participants spoke of being motivated to engage in GHPs by a desire "to help" the local communities, or "to teach" the local healthcare providers, embedded within this implied sense of responsibility, was also an implied sense of superiority (Burgess et al., 2014).

In an insightful application of Andreotti’s (2006) framework on "soft versus critical global citizenship education”, Burgess et al. (2014) concluded that the majority of participants were motivated to engage in overseas practicums by what Andreotti calls, a 'soft' approach to global citizenship (2006). While I will explore, in greater depth, the usefulness of Andreotti’s framework (2006) in the recommendations section of this chapter, 'soft' approaches to global citizenship education can be broadly categorized into pedagogical methods which, for a number of reasons, fail to encourage learners' critical literacy, or awareness of notions such as "power, voice, and difference” (Andreotti, 2006, p. 49). To a similar end, 'soft' motivations for global citizenship, such as those evidenced in this study as well as in Burgess et al.'s qualitative exploration (2014), are largely grounded in moral imperatives for action (Andreotti, 2006). For instance, there were times when our participants were motivated to engage in GHPs by a desire "to teach" local healthcare providers or to capitalize on an opportunity to "give back" and "help" more impoverished nations in the global South.

This is not to say, however, that such 'soft' motivations for global citizenship can not serve as an important foundation for engaging in GHPs, or as a beginning point for fostering 'critical' global citizenship (Andreotti, 2006; Burgess et al., 2014). Indeed, it would be discouraging, and 
perhaps, insensitive, to suggest otherwise. However, in opposition to 'the good' that what was initially intended, actions grounded solely in moral imperatives for action can end up contributing to the reinforcement of existing power imbalances as well as to the sustained vulnerability of communities on the receiving end of such actions (Dobson, 2006). Therefore, as Andreotti (2006) explains, fostering a critical perspective in global citizenship education, is a helpful, and indeed, necessary, approach to facilitating individuals' understanding of global issues:

[F]or the creation of an ethical relationship with learners (and with the South), the development of critical literacy becomes necessary. I conceptualise critical literacy as a level of reading the word and the world that involves the development of skills of critical engagement and reflexivity: the analysis and critique of the relationships among perspectives, language, power, social groups, by the learners. Critically, in this context, does not refer to the dominant notion that something is right or wrong, biased or unbiased, true or false. It is an attempt to understand origins of assumptions and implications (p. 49). This study's findings suggest that participants were largely motivated to attend GHPs from moral imperatives for engagement; opportunities "to help", or "to give back" to the local communities. While these motivations are well intentioned and can lay a beginning basis for engagement in overseas practicums (Andreotti, 2006; Burgess et al., 2014), such motivations are, and can be construed as, paternalistic and colonial in an already colonized context. It is important to note, however, that not all forms paternalism are morally wrong or ethically impermissible (Dawson, 2011). Indeed, some paternalistic actions (e.g., regulation of the food industry to combat rising rates of obesity) can be incredibly positive in the context of public health and safety more broadly (Dawson, 2011). However, in the context of this study's findings, the 
morally problematic form of paternalism was embedded in the participants' implicit assumptions of the 'good' they were intending to provide during these experiences. For instance, what implicit beliefs about 'progress' did these students hold? Did they impose a culture of healthcare that aligned with the wants, needs, and desires of the local healthcare system? And further, did this imposition of culture align with the wants, needs, and desires of the local patients, families, and other community members? With this in mind, it is critical that nurse educators explore and make explicit nursing students’ motivations and expectations for engaging in GHPs and seek to foster - early on in the preparation for GHPs - critical approaches to global citizenship education (Andreotti, 2006).

\subsubsection{The role of misaligned expectations in nursing students' experiences of moral uncertainty during GHPs}

Narratives which amplified participants’ experiences of moral uncertainty often revealed a growing disjuncture between what participants had expected to occur during their GHP, and what was experienced in reality. Much like the feelings of anger, frustration, dispiritedness, and guilt that are characteristic of nurses’ experiences of moral distress (Pauly, 2012; Rodney \& Starzomski, 1993; Rodney \& Varcoe, 2001; Rushton, 1992; Yeo \& Ford, 1996), misaligned expectations in the context of this study contributed to nursing students' experiences of moral uncertainty, and subsequently to emotional responses indicative of guilt, frustration, and helplessness. In the following section, I will discuss how misaligned expectations were woven throughout the four main themes typifying participants' experiences of moral uncertainty, with a particular focus on the misaligned expectations embedded within the sub-themes encompassed by cultural collisions in healthcare, blurring of boundaries: Scope of practice issues, and connectedness. 
Firstly, in the theme, cultural collisions in healthcare, participants were struck by what was perceived as a quelled, or slowed, approach to care. Times when the local healthcare providers' response was perceived, by the GHP participants, to be less urgent, less relational, and at times, lacking in resiliency and resourcefulness. Interestingly, however, experiencing a quelled approach to care sat in direct juxtaposition to what many of the participants had expected to learn from, and observe, during their GHP. In accordance with the existing literature (Gower et al., 2016), a number of participants were motivated to participate in a GHP by the allure of developing a sense of resourcefulness and resiliency as a result of practicing in a healthcare context that was perpetually resource constrained. However, when expectations of developing one's sense of resourcefulness were met by a healthcare culture that was perceived to be lacking in innovative and action-oriented approaches to healthcare, participants experienced, first hand, the frustration, anger, and unease which resulted from misaligned expectations.

A similar disjuncture was evident in the theme, blurring of boundaries: Scope of practice issues. As I discussed in an earlier section, many participants were motivated to engage in a GHP as a means to "giving back" to those less fortunate, or "to help" the local communities in some healthcare related capacity. In some instances, such 'soft' motivations for global citizenship (Andreotti, 2006) fueled participants' experiences of guilt when one’s expectations and/or motivations could not, or were not, operationalized in the context within which participants found themselves. For example, when one's expectation "to help" was replaced with feelings of helplessness in being uncomfortable or unable to provide care that was beyond the scope or practical means of a Canadian nursing student, feelings of guilt arose. Such feelings of guilt and helplessness are evident in the following participant reflection: 
So there were a number of times where I did step back and said like, no, I don’t feel comfortable. And I felt a little guilty because you know, here I am having come so far to you know, provide some sort of help to people, and you know, I’m just taking a step back and being like, no, I’ll just watch for my own learning.

Lastly, in the theme, connectedness, misaligned expectations, and the disjuncture that ensued, stemmed from the belief that participants would be embraced by the local communities as if they were their "brothers and sisters". While this was not an expectation that was overtly stated by the majority of participants, a number of participants did vocalize that they expected to be welcomed into the community in a spirit of celebration, appreciation, and a sense of belonging. When resistance to this expectation was met through a perceived unwillingness - on the part of the hosts - to engage and participate in an exchange of knowledge or friendship with the GHP participants, feelings of resentment and frustration were fostered. Interestingly, while this idea of a 'saviour complex' has been addressed by the theoretical literature concerned with global citizenship (Andreotti, 2006), it has yet to be - to the best of my knowledge - amplified in the empirical literature on the benefits and challenges of GHPs in nursing.

Interestingly, however, participants' experiences of moral uncertainty were not always rooted in misaligned expectations. Rather, at times, moral uncertainty ensued from what I will call an alignment of expectations. For example, for those participants who were motivated to engage in GHPs for reasons related to skill-building opportunities - a factor that has identified in nursing students' motivations for GHPs (Kent-Wilkinson et al., 2015) - the fulfillment of such expectations, while accomplished, nevertheless contributed, in some instances, to participants' experiences of moral uncertainty. For instance, after reflecting on occurrences of practicing 
beyond one's scope of practice during times where the potential for harm was evoked, one GHP participant reflected, "I wish I had done a little bit less".

As reflected in the anecdotes shared above, findings suggest that nursing students’ experiences of moral uncertainty were often precipitated by expectations. In some instances, participants experienced uncertainty in the disjuncture that resulted from misaligned expectations. Other times, uncertainty was triggered by the fulfillment of expectations, whereby GHP participants accomplished practices which they had intended, or hoped, to achieve, but were left to grapple with the consequences of their actions. However, irrespective of the 'alignment' of the expectations, experiences of moral uncertainty ensued.

\subsubsection{Redressing experiences of moral uncertainty in the context of GHPs}

Despite the feelings of frustration, anger, guilt, and helplessness that stemmed, in part, from participants' experiences of moral uncertainty, I am not suggesting that GHPs should be void of any opportunities for nursing students to grapple with issues of morality and ethics. Indeed, there is a growing body of literature which suggests that by grappling with issues of morality - as occurs in instances of moral distress and moral uncertainty -, one is afforded an opportunity to engage in personal reflection and advocacy, thereby contributing to positive personal growth (Lützén \& Kvist, 2012; Rodney, 2017). However, the process of how we wrestle with the frustration, anger, helplessness, and dispiritedness that often accompanies positions of moral distress (Erlen \& Frost, 1991; Pauly, 2012; Rodney \& Starzomski, 1993; Rodney \& Varcoe, 2001; Rushton, 1992; Yeo \& Ford, 1996) and moral uncertainty (Cohen \& Erickson, 2006; Hamric, 2000), as well as an examination of why nursing students are experiencing such uncertainty during GHPs, is of paramount importance. The importance of understanding these dimensions of nursing students' experiences of moral uncertainty will be addressed below. 
Experiences of moral uncertainty were evident through this study’s findings. Participants exhibited an array of emotional responses to such experiences of moral uncertainty, ranging from feelings of frustration and helplessness, to anger, dispiritedness, and fear. Some participants were able to resolve experiences of moral uncertainty through personal reflections or through discussions with their peers, instructors, and family members. However, for others, the moral uncertainty remained unresolved.

A number of negative consequences have been linked to nurses' experiences of moral distress (Rushton, 2017). Moral distress has been associated with nurse attrition (Huffman \& Rittenmeyer, 2012; Pauly, 2012), emotional withdrawal from patients (Meltzer \& Huckabay, 2004; Ohnishi et al., 2010), a diminished self-worth (Huffman \& Rittenmeyer, 2012), and expressions of emotional distress, such as bouts of anger, disgust, and frustration (Huffman \& Rittenmeyer, 2012; Radzvin, 2011; Rushton, Caldwell, \& Kurtz, 2016). Unresolved moral distress has been shown to manifest in a sense of powerlessness (Rushton, 2017). Furthermore, prolonged, and/or repeated exposure to moral distress, without adequate intervention or resolve, has been shown to result in moral compromise and experiences of moral residue (Epstein \& Hamric, 2009; Rodney, Brown \& Liaschenko, 2004; Webster \& Baylis, 2000). Moral residue has been defined in the literature as "what we carry with us when we knew how we should act but were unwilling and/or unable to do so” (Rodney, Brown \& Liaschenko, 2004, p. 163).

A number of insightful strategies for mitigating, redressing, and preventing the negative consequences associated with moral distress have been identified in the literature (Beumer, 2008; Burston \& Tuckett, 2013; Hanna, 2005; Meaney, 2002; Pendry, 2007; Kalvemark et al., 2004). In a comprehensive overview of the existing literature on moral distress, Burston and Tuckett (2012) found that interventions to combat moral distress could be broadly categorized into two 
main themes: individualistic approaches or collaborative approaches. Perhaps unsurprisingly, individualized approaches encouraged the development of one's own coping strategies to combat moral distress (Burston \& Tuckett, 2012). Oftentimes, this was fostered through periods of critical self-reflection (Hanna, 2004), or through the development of ethical decision-making skills (Malloy et al., 2009; Meaney, 2002). Conversely, collaborative approaches to working through experiences of moral distress have suggested, amongst other things, the importance of interdisciplinary staff meetings and/or ethics rounds (Rice et al., 2008). Most recently, the solution-oriented and self-reflexive concept of moral resilience has emerged as a possible response to moral distress (Rushton, 2017). Moral resilience refers to one’s "capacity to sustain, restore, or deepen [original emphasis] her or his integrity in response to moral complexity, confusion, distress, or setbacks” (Rushton, 2017, p. S13).

In the context of my study's findings, nursing students often redressed feelings of moral uncertainty through periods of self-reflection, through formal and informal discussion groups or debriefing sessions, and by retreating to familiar environments (e.g., participants' lodging) and familiar rituals (e.g., watching Western television shows). Interestingly, however, study findings indicate that the way participants processed their moral uncertainty, or sought to redress feelings of distress, frustration, or discomfort, contributed, in part, to unintended and undesirable consequences. For instance, when participants grappled with perceptions of a quelled approach to care, resolution of moral uncertainty often resulted from thought processes and reflections which attributed feelings of moral uncertainty to having witnessed a less innovative, less resourceful, and less caring healthcare system in action. This was exemplified when participants attributed perceptions of a less-urgent approach to care to a difference in the value that life in the host countries held. This was also demonstrated when perceptions of a lack of urgency or 
responsiveness to patients' needs were attributed to a healthcare system that was lacking in resourcefulness or a caring approach to nurse-patient relationships. Other times, participants resolved moral uncertainty by attributing it to irreconcilable differences between the healthcare system participants were familiar with, and the one participants found themselves in during GHPs. Thus, in order to avoid such uncertainty in the future, one participant indicated, ... and one of the things I did decide if I ever did do foreign aid again...is that I want to work for an organization that I understand, and [that] the culture of the organization aligns with the culture I'm used to...so that I understand the hierarchy and I can be like this is wrong.

It is not to say, however, that reflexive processes which seek to redress individuals’ experiences of moral uncertainty are not a necessary component of GHPs. Indeed, I would argue that the ability to reflect on feelings of moral uncertainty is an integral and indispensable part of GHPs in nursing. However, reflexive processes which fail to critically examine one’s own positioning and assumptions alongside the historical, social, and political context within which this moral uncertainty develops, can, as suggested above, result in the reinforcement of negative stereotypes, as well as a diminished desire to work outside of one's cultural frame of reference. Therefore, as a means to mitigating such unintended and undesirable consequences of GHPs, in the following section I will discuss the importance of grounding GHPs in post-colonial, crosscultural, and critical lenses; and in particular, the importance of assessing and facilitating the development of nursing students' social and moral consciousness both prior to, during, and upon return home from GHPs (Giddings, 2005). 


\subsubsection{Moral uncertainty: An opportunity to facilitate the development of students' critical and ethical decision-making}

As Rodney, Pauly and Burgess (2004) remind us, “[s]ince morality is acted out in a cultural context, it is critical to try and engage in a dialogue with others about their cultural beliefs and practices before we pass judgment or implement decisions” (p.83). Findings from this study indicate that a number of participants began to grapple with the weight and importance that cultural beliefs and practices have on the delivery of healthcare services in the global context. However, for many, the way in which participants grappled with or made meaning of their moral uncertainty also showed a need for a more nuanced and critical approach to reflecting on, and making space for, cross-cultural interactions in healthcare. Like other contextualist approaches to analyzing ethical issues in practice, such as feminist ethics (see: Sherwin, 1992), cross-cultural ethics invites us to consider the context and circumstances within which issues of morality and ethics arise in practice (Rodney, Pauly, Burgess, 2004). For instance, in the context of this study's findings, when a participant was grappling with how best to accept and process feelings of moral uncertainty upon witnessing the care of a miscarried infant and the treatment of her mother, instead of labelling it as a "horrible...way to handle [it]", a cross-cultural approach to analysis would invite the participant to consider other "morally relevant features in the situation" (Rodney, Pauly, \& Burgess, 2004, p. 81) that may have been overlooked as a result of an ethnocentric, or in this case, Western, approach to ethical analysis. In the context of this example, such "morally relevant features" (p. 81) might have included an exploration of the local healthcare providers' beliefs around caring for still-born infants, or a broader examination of the culture of death and dying in the local community. 
Furthermore, post-colonial theory has been identified as a helpful adjunct to cross-cultural ethics (Brown, Rodney, Pauly, Varcoe, \& Smye, 2004). Just as cross-cultural and other contextualist approaches to ethical analysis invite us to consider additional "moral aspects that a different theoretical lens might tend to miss or distort” (Rodney, Pauly, Burgess, 2004, p. 78), post-colonial theory takes us a step further, and asks us to critically (re)consider how dominant discourses have been constructed (Anderson \& McCann, 2002; Brown et al., 2004). As McConaghy (1997) posited, a willingness to engage with post-colonial perspectives ...is a sign that we are now more aware of our historical locatedness, less sure of the rightness of our policy decisions, more alert to the possibility that our decisions may be colonizing rather than decolonizing in their consequences, more able to be responsive to new situations of disadvantage and more able to correctly analyze and redress the specifics of local oppressions (p. 86, as cited in Brown et al., 2004).

It is important to note, however, that 'post', in post-colonial, does not signify an end to colonialism. Rather, it is a lens and reflexive process by which explicit attention is drawn to colonialism "as it existed and exists" (Brown et al., 2004, p. 142) around the world, and encourages individuals to reflect on the socio-historical conditions which contribute to the sustained marginalization of distinct populations (Brown et al., 2004; Varcoe, 2004).

Findings from this study indicate that nursing students’ experiences of moral uncertainty were influenced, in part, by motivations, expectations, and reflexive processes which could benefit from critical and contextualized ethical theories, such as post-colonial and cross-cultural ethics. These findings echo previous calls for the inclusion of critical pedagogies in GHPs that have been made in the theoretical literature dedicated to exploring the ethics of international practicums (Hanson, Harms, \& Plamondon, 2001; Levi, 2009; Racine \& Perron, 2012). In the 
following section, I will look to expand on these findings, by engaging in a discussion on the usefulness of Giddings’ Dialectical Model of Social Consciousness (2005) as a means to analyzing students' awareness of social injustice during GHPs, and in particular, students' awareness of social injustice when processing experiences of moral uncertainty. For the purposes of this discussion, social consciousness is defined as "personal awareness of social injustice" (Giddings, 2005, p. 224).

Giddings’ Dialectical Model of Social Consciousness (2005) highlights, from a postcolonial lens, how individuals respond to social injustice - in both their personal lives and in the lives of others - through either an "acquired social consciousness”, an “awakened social consciousness”, or an “expanded social consciousness” (Giddings, 2005). According to Giddings, when individuals are positioned in an "acquired social consciousness”, power imbalances, health and social disparities, and the maltreatment of others is simply, and often uncritically, accepted (2005). From a position of an "awakened social consciousness", however, individuals actively acknowledge and seek to disrupt legacies of social inequities, challenging "taken-for-granted assumptions about his or her identity or assiduously monitors the dominant cultures for unjust practices” (Giddings, 2005, p. 230). Lastly, from a position of an "expanded social consciousness”, Giddings’ suggests that individuals uphold a more nuanced understanding of the relationship between privilege and oppression and take a more balanced approach to disrupting harmful legacies of injustice. From this position, individuals choose to engage in critically chosen actions only when one's involvement has been thoughtfully and critically considered (Giddings, 2005).

Narratives of moral uncertainty amplified in this study's findings revealed responses to social injustice that spanned all three positions of Giddings' Dialectical Model of Social 
Consciousness (2005). As Giddings’ model suggests, participants transitioned within and between the three positions of social consciousness in what can be described as a fluid-like manner (2005). Most often, participants entered their GHP from a position of an "acquired social consciousness" and then transitioned, to varying degrees, into either an "awakened" or “expanded social consciousness” (Giddings, 2005), depending on the context and experiences they encountered.

Perhaps unsurprisingly, participants' positioning in this study influenced how they grappled with, and were exposed to, experiences of moral uncertainty. For instance, participants who articulated a position of an "acquired social consciousness" oftentimes failed to critically examine the socio-historical contexts within which their experiences of moral uncertainty arose (Giddings, 2005). This was evident in the context of this study's findings when the meaning that participants ascribed to their moral uncertainty perpetuated negative and undesirable stereotypes of the host communities. Furthermore, in some situations, participants' experiences of moral uncertainty may have resulted from the ascribed nature of their social consciousness (Giddings, 2005). This was evident when participants experienced moral uncertainty as a result of not being welcomed into the host communities in a spirit of celebration, or when one participant experienced moral uncertainty upon witnessing the care of a miscarried infant and the treatment of her mother. In this latter example, moral uncertainty was perpetuated by the participant's belief that there was an appropriate and right way to behave in this context, and when this 'right' way to respond did not occur, feelings of moral uncertainty ensued. According to Giddings, such rigid and decontextualized approaches to ethical decision-making are common in individuals who operate from positions of an "acquired social consciousness" (2005). 
For participants who voiced a more "awakened” or "expanded” position of social consciousness (Giddings, 2005), experiences of moral uncertainty resulted in the development of a more nuanced understanding of issues related to social justice, rather than a perpetuation of the status quo, and encompassed within that, the perpetuation of negative stereotypes. This was exemplified when participants grappled with heightened positions of power; questioned links between GHPs and neocolonialism; sought to understand the broader social, economic, and political contexts which influenced the lives of individuals from the host nations; and when one participant grappled with the connection between privilege and voyeurism when a walking tour of the local community turned - from the participant's perspective - into an exercise in objectification:

...the instructor was trying to say, it's like someone walking down your street, and I was like, it feels different than that because we are walking down their street to be like, look how sad it is here. That's not an appropriate thing to do to someone else's home, but then at the same time, is it worth it, if all of the people that do international practicums then have a widened world view?

While this participant remained in a place of uncertainty - questioning the objectives of GHPs more broadly - this critical perspective indicates a more nuanced understanding of injustice, and in turn, suggests the development of a more critical approach to global citizenship (Andreotti, 2006).

The usefulness of Giddings’ Dialectical Model of Social Consciousness (2005), as well as the importance of facilitating the development of nursing students' social consciousness during GHPs, has not gone unidentified in the empirical literature. In an exploration of the nature of nursing students' learning during GHPs, as well as an investigation into the long-term benefits of 
such practicums, Reimer Kirkham, Van Hofwegen and Pankratz (2009) found that GHPs provided nursing students with meaningful opportunities to engage with, and facilitate the development of, one's social consciousness (2009). However, the sustained integration of this heightened social consciousness into students' personal and professional lives upon return home from GHPs, proved to be a more challenging task (Reimer Kirkham et al., 2009). In response to the important questions about the long-term benefits of GHPs that these findings raised, Reimer Kirkham et al. (2009) proposed a Preliminary Framework for International Learning Experiences. With an added emphasis on "partnerships and equity for all stakeholders" (p. 12), Reimer Kirkham et al.'s framework seeks to provide a starting point for fostering the sustained development of students' social consciousness during GHPs, all the while encouraging careful consideration of the more harmful effects of international practicums (2009).

Findings from this study suggest that the positioning of nursing students’ social consciousness influenced their experiences of moral uncertainty both prior to, during, and upon return home from their GHP. Furthermore, despite narratives which were indicative of a heightened social consciousness, and a more nuanced understanding of social justice issues, a number of participants grappled with moral uncertainty from standpoints indicative of an ascribed, or less critical, approach to social consciousness (Giddings, 2005). Unfortunately, such positioning had the potential to result in the reinforcement of negative and undesirable stereotypes of the host communities as participants sought to redress feelings of moral uncertainty. This was exemplified when participants attributed differences in the approaches to care, and in particular, a less action-oriented approach to care, as resulting from a culture where the meaning of life held a diminished, less acute, value. Other times, negative stereotypes were perpetuated when participants attributed a perceived lack of urgency and resourcefulness to a 
healthcare culture that simply “didn’t have the same drive to really change and look for ways to improve things”. Therefore, in the unfamiliar, and oftentimes disorienting, environments students find themselves in during GHPs, sustained and dedicated attention to facilitating the development of nursing students' social consciousness is imperative. Strategies for facilitating the development of nursing students' social consciousness, as well as encouraging the use of contextualized approaches to ethical analysis, will be discussed in the recommendations section below.

\subsection{Recommendations}

In light of this study's findings, this section presents recommendations for practice, education, and future research, with an emphasis on fostering students' ethical development prior to, during, and upon return home from undergraduate nursing GHPs. I will begin by discussing recommendations for nurse educators in supporting nursing students in advance of GHPs. I will then provide recommendations for nurse leaders and nurse educators responsible for supporting nursing students prior to, during, and upon return home from their GHPs. I will then conclude with recommendations for future research.

\subsubsection{Education}

This study's findings indicate that nursing students encountered times of moral uncertainty, and less frequently, moral distress, during their GHPs. Narratives of participants' experiences of moral uncertainty highlighted varying depths of critical reflection. Variations ranged from understandings of social justice concomitant with an "awakened" or "expanded” social consciousness (Giddings, 2005), to more superficial reflections representative of an "ascribed social consciousness” (Giddings, 2005). As I briefly discussed in Chapter One, several strategies for facilitating the sustained development of nursing students' critical and ethical decision- 
making during GHPs have been suggested (Hanson et al., 2011; Leininger, 1998; Levi, 2009; Racine \& Perron, 2012). For instance, Hanson et al. (2011) call for the introduction of pedagogical approaches that are designed to build students' "capacity to critically analyze and challenge the institutional and structural inequalities embodied in Western models of development and medicine” (p. 179). However, beyond the importance of focused education prior to, during, and upon return home from GHPs, findings from this study suggest that a greater emphasis should be placed on ethics education more broadly, with a particular focus on building nursing students' capacity for ethical decision-making and navigating experiences of moral uncertainty and moral distress. Furthermore, participants' narratives of moral uncertainty suggest that the integration of critical and transformative pedagogies throughout the entirety of nursing students' undergraduate education may be beneficial.

As discussed in an earlier section of this chapter, Andreotti's (2006) framework on "soft versus critical global citizenship education” may be a helpful approach to facilitating individuals’ understandings of global issues from critically located and self-reflexive standpoints. While the framework is not targeted at undergraduate nursing education specifically, the usefulness of Andreotti’s (2006) framework throughout the continuum of undergraduate nursing education should not be overlooked. Using Andreotti’s approach to 'critical' global citizenship education (2006), individuals are asked, amongst other things, to reflect on their motivations for engaging in practices that seek to redress global injustices, such as health and social inequities. In juxtaposition to 'soft' motivations for global citizenship (see: Table 3), individuals operating from 'critical' global citizenship perspectives are motivated to attend to global issues, not by moral imperatives for action- as has been articulated in the existing literature (Burgess et al., 
2014), as well as in this study's findings - but rather, by political and ethical imperatives for engagement (Andreotti, 2006).

Furthermore, 'critical' approaches to global citizenship education provide the space, knowledge, and encouragement to consider the "causal responsibility" of the global injustices or disparities that these global health initiatives are likely trying to redress (Andreotti, 2006; Dobson, 2006). Thus, where 'soft' global citizenship education sees the role of 'ordinary' individuals in redressing global injustices as "some individuals are part of the problem, but ordinary people are part of the solution” (Andreotti, 2006, p. 47), 'critical' global citizenship education encourages learners to grapple with the idea that "we are all part of the problem and part of the solution” (Andreotti, 2006, p. 47). While such framing is important for preparing nursing students for GHP-like experiences, facilitating the development of students' critical global citizenship throughout the continuum of undergraduate nursing education has the potential to positively influence the delivery of ethical and equity-centred care both at home and abroad. 
Table 3. 'Soft' versus 'critical' global citizenship education

\begin{tabular}{|l|l|l|}
\hline & $\begin{array}{l}\text { Soft global citizenship } \\
\text { education }\end{array}$ & $\begin{array}{l}\text { Critical global citizenship } \\
\text { education }\end{array}$ \\
\hline Problem & Poverty, helplessness & Inequality, injustice \\
\hline $\begin{array}{l}\text { Nature of the } \\
\text { problem }\end{array}$ & $\begin{array}{l}\text { Lack of “development”, } \\
\text { education, resources, skills, } \\
\text { culture, technology, etc. }\end{array}$ & $\begin{array}{l}\text { Complex structures, systems, } \\
\text { assumptions, power relations, and } \\
\text { attitudes that create and maintain } \\
\text { exploitation and enforced } \\
\text { disempowerment and tend to } \\
\text { eliminate difference }\end{array}$ \\
\hline Basis for caring & $\begin{array}{l}\text { Common humanity/being } \\
\text { good/sharing and caring. } \\
\text { Responsibility FOR the other } \\
\text { (or to teach the other) }\end{array}$ & $\begin{array}{l}\text { Justice/complicity in harm. } \\
\text { Responsibility TOWARDS the } \\
\text { other (or to learn with the other) - } \\
\text { accountability }\end{array}$ \\
\hline Ground for acting & $\begin{array}{l}\text { Humanitarian/moral (based on } \\
\text { normative principles for } \\
\text { thought and action) }\end{array}$ & $\begin{array}{l}\text { Political/ethical (based on } \\
\text { normative principles for } \\
\text { relationships) }\end{array}$ \\
\hline $\begin{array}{l}\text { Understanding of } \\
\text { interdependence }\end{array}$ & $\begin{array}{l}\text { We are all equally } \\
\text { interconnected, we all want the } \\
\text { same thing, we can all do the } \\
\text { same thing }\end{array}$ & $\begin{array}{l}\text { Asymmetrical globalization, } \\
\text { unequal power relations, Northern } \\
\text { and Southern elites, imposing own } \\
\text { assumptions as universal }\end{array}$ \\
\hline $\begin{array}{l}\text { Notes: Permission to reproduce granted by Caroline Burgess (May 2018). Original permission } \\
\text { to reproduce granted by Dr. Andreotti (December 2012) from Andreotti (2006) (pp. 46-48). }\end{array}$ \\
\hline
\end{tabular}

Additionally, in 2015, the Canadian Coalition for Global Health Research (CCGHR) released the Principles for Global Health Research (CCGHR, 2015). Grounded in a concern for health equity, six interrelated principles for conducting global health research were proposed: humility, responsiveness to causes of inequities, commitment to the future, inclusion, authentic partnering, and shared benefits (CCGHR, 2015). While these principles are primarily geared toward conducting equity-centred research (CCGHR, 2015), their applicability to GHPs, and undergraduate nursing education more broadly, should not be dismissed. Of particular relevancy, is the recognition that research, and indeed, the delivery of healthcare services in general, are not "conducted outside of the frame of power relations that creates health inequities" (Cleaver, Magalhaes, Bond, Polatajko, \& Nixon, 2016, p. 1023; CCGHR, 2015). Thus, from this 
perspective, students are called to critically reflect on their role in redressing, but also creating and maintaining health and social inequities.

This study's findings also indicate that participants experienced considerable amounts of moral uncertainty upon engaging in practices beyond one's scope of practice. While similar findings have been identified in the empirical ethics literature dedicated to exploring the ethical challenges of GHPs encountered by medical students (Elit et al., 2011; Abedini et al., 2012), to the best of my knowledge, this is a novel contribution to the empirical nursing literature. Such findings raise the importance of educating nursing students on the weight of scope of practice, as well as the importance of adhering to the College of Registered Nurses of British Columbia's Professional Standards for Registered Nurses and Nurse Practitioners (2013), the Canadian Nurses Association's Code of Ethics (2017), and the document outlining undergraduate nursing students’ Scope of Practice at UBC Okanagan’s School of Nursing (2015).

Lastly, nursing schools across Canada are working to respond to the Truth and Reconciliation Commission of Canada: Calls to Action, including Call to Action 24, which mandates all Canadian medical and nursing schools to "require all students to take a course dealing with Aboriginal health issues, including the history and legacy of residential schools, the United Nations Declaration on the Rights of Indigenous Peoples, Treaties and Aboriginal rights, and Indigenous teachings and practices", with preparation in "intercultural competency, conflict resolution, human rights, and anti-racism” (Truth and Reconciliation Commission of Canada, 2015, p.7). In addition, the College of Registered Nurses of BC (CRNBC), the provincial regulatory body which recognizes baccalaureate and nurse practitioner education programs in BC, has committed to work in partnership with the First Nations Health Authority and 22 other provincial health regulators to collectively "review how cultural safety and humility can be better 
integrated into quality assurance programs, complaints processes and post-secondary programs” (CRNBC, 2017). The decolonizing work in BSN teaching and learning that CRNBC, nurse educators and nursing students undertake with Indigenous Elders, knowledge keepers, communities and organization can inform undergraduate global health curriculum and support BSN students' preparation for, critical reflection during, and debriefing after GHPs.

\subsubsection{Pre-departure training}

Focused education tailored uniquely to students who decide to pursue an international experience, such as a GHP, evolved out of calls for greater transparency and ethical delivery of international clinical experiences (Pinto \& Upshur, 2000; Wallace \& Webb, 2014; Hanson et al., 2011; Petrosoniak, McCarthy, \& Varpio. 2010). Pre-departure training, as it is referred to in the literature, denotes the training students receive prior to GHPs to help prepare them for learning and practicing in the global context (Wallace \& Webb, 2014). Specifically, pre-departure training seeks to build "trainee competence in the skills necessary to maximize learning while also minimizing harm to themselves and the communities in which they study” (Anderson et al., 2012, p. 206). In an exploration of pre-departure training in Canadian medical schools, Anderson et al. (2012) found that while 16 of the 17 Canadian medical schools offered some form of predeparture training, significant differences in the structure, content, facilitation, and duration of such programs existed. Despite this variance, a recent review of recommended pre-departure training practices found that the type of education delivered during the training could be broadly categorized into six themes: global health knowledge; ethics, introspection, and critical thinking; cultural competency; cross-cultural communication; placement-specific knowledge; and personal health and safety (Bessette \& Camden, 2017). 
While it was beyond the scope of this study to explicitly explore the training nursing students received prior to departure, findings suggest that a more robust approach to predeparture training is needed. Broadly speaking, nursing students within this study grappled with issues related to professionalism, scope of practice, power differentials, cultural competency, and sustainability. Interestingly, many of these topics are located within the pre-departure practice themes identified by Bessette and Camden (2017). Therefore, in addition to embedding critical pedagogies across undergraduate nursing curriculum, nurse educators responsible for preparing students for GHPs should look to draw upon existing research detailing pre-departure practices for health sciences students. A particular emphasis should be placed on incorporating practices which seek to mitigate ethical challenges that may arise during GHPs. To that end, several freely available online curricula dedicated to preparing trainees for ethical challenges in global health exist and would make excellent additions to pre-departure training. Of particular interest may be the online modules created by the University of British Columbia entitled, "Ethics of International Engagement and Service-Learning Project” (2011), as well as the case-based curriculum - "Ethical Challenges in Short-Term Global Health Training” - created by DeCamp, Rodriguez, Hecht, Barry and Sugarman (2013). Furthermore, a number of innovative approaches to preparing students for GHPs have recently been identified in the literature. Of particular interest is the use of simulation-based training to prepare students for the ethical challenges inherent in international healthcare related practicums (see: Asao, Lewis, Harrison, Glass, \& Penich Brock, 2017; Logar et al., 2015). Asao et al. (2017) examined the usefulness of simulation-based training in preparing medical students for GHPs and found that "[i]n the absence of systematic and substantive guidelines regarding ethical challenges in global health work, incorporation of simulations within broader global health curricula may improve trainees' 
ability to deal with the ethical challenges they will inevitably encounter” (p. 7). Lastly, important work in the area of global health competencies has been undertaken by the Consortium of Universities for Global Health (CUGH). The Consortium's Global Health Education Competencies Toolkit (2017) may be a helpful guiding document for both students and instructors alike as they prepare for their GHP experience.

\subsubsection{Practice}

\subsubsection{Clarifying program objectives}

This study's findings suggest that much of the moral uncertainty nursing students experienced during GHPs was related to expectations. Most often, moral uncertainty arose from misaligned expectations; times when nursing students' motivations and expectations for participating in a GHP, did not align with what was experienced in reality. Other times, it was the actualization of nursing students' motivations and expectations for GHPs that propelled, and subsequently prompted, students’ experiences of moral uncertainty. Further still, however, participants' narratives of moral uncertainty revealed a perceived lack of clarity - on the part of host healthcare providers - about the role of GHP participants in the clinical setting. Whether moral uncertainty resulted from misaligned expectations, or not, such findings suggest a need for revisiting and clarifying the objectives of GHPs.

In 2010, the Working Group on Ethics Guidelines for Global Health Training (WEIGHT) developed a set of ethics and best practice guidelines for training experiences in global health (Crump \& Sugarman, 2010). Embedded within these guidelines for trainees, institutions, and sponsors of GHP-like experiences, was a call for trainees and institutions to "recognize that the primary purpose of the experience is global health learning and appropriately supervised service" (Crump \& Sugarman, 2010, p. 1180). In order to ensure that such a mandate is met, and perhaps 
more importantly, to ensure that all parties involved in the GHP "derive mutual, equitable benefits" (p. 1179), explicit discussions on the responsibilities and expectations of both the sending and host institutions are of paramount importance and should be revisited on a periodic basis (Crump \& Sugarman, 2010).

Lastly, it is important to emphasize that GHP students from the sending institutions also have a responsibility in communicating the objectives, expectations, and goals of GHPs to the local healthcare providers they are working alongside. This should be done prior to engaging in appropriately supervised practice (Crump \& Sugarman, 2010), thereby potentially avoiding situations where students may feel compelled or pressured into engaging in practices beyond their ability and scope of practice.

\subsubsection{Practicum support}

Participant narratives in this study suggest that nursing students experienced situations within their GHP that challenged their thinking ethically, emotionally, and morally. The moral uncertainty that amounted from such experiences encouraged students to engage in numerous forms of debriefing. Participant narratives highlighted the importance of formal debriefing periods, the helpfulness of informal discussion groups with fellow nursing students, as well as the integral, but oftentimes, overlooked, importance of debriefing sessions upon return home from GHPs. However, findings also indicate that the way participants processed feelings of moral uncertainty contributed to unintended, and at times, negative, consequences. This raises the importance of facilitating safe, yet critically located spaces for students to process feelings of discomfort, frustration, and confusion that may arise during GHPs.

The importance of clinical debriefing sessions has been noted in the nursing literature (Galvin, Suominen, Morgan, O’Connell, \& Smith, 2015; Megel, Nelson, Black, Vogel, \& 
Uphoff, 2013; Potgeiter, 2012). Cited benefits include a reduction in clinical anxiety after particularly challenging clinical encounters (Galvin et al., 2015), as well as an opportunity to foster the development of nursing students' critical thinking and ability to make more sound nursing judgments (Megel et al., 2013; Potgeiter, 2012). However, in order to facilitate stimulating and transformative debriefing sessions, instructors must be well versed in the knowledge and skills required to help students learn from and grapple with encounters that are both clinically and ethically challenging. In the context of our findings, a foundation in critical pedagogies (i.e., post-colonial theory, global health ethics, cross-cultural ethics, and cultural humility) would be helpful. This is particularly evident from the standpoint of facilitating development of nursing students' social consciousness (Giddings, 2005) and “critical” global citizenship (Andreotti, 2006). It is also evident when helping nursing students to process their experiences of moral uncertainty and moral distress in a constructive, safe, and critically located way. Ultimately, nursing programs overseeing the facilitation of GHPs need to provide clinical instructors with the resources required to develop and build upon this foundational body of knowledge.

In the context of this study's findings, nursing students' experiences of moral uncertainty often arose out of conflicts between the values, beliefs, and practices of the host nations, and the values, beliefs, and assumptions of the GHP participants. In order to mitigate some of the clashes between cultures both in the clinical setting and community setting, the inclusion of a cultural broker has been suggested (Underwood, Gleeson, Konnert, Wong, \& Valerio, 2016; Vance, Vaiman, \& Anderson, 2009). The cultural broker can provide visiting students and faculty with insight into relevant political, historical, and social influences (Leffers \& Mitchell, 2011), as well as important insight into cultural practices, such as dress, communication styles, and health 
beliefs (Underwood et al., 2016), thus helping to foster students' cultural competency (Crump \& Sugarman, 2010). In an effort to transition to more equitable partnerships, whereby host communities are involved in all aspects of the planning and execution of GHPs (Crump \& Sugarman, 2010), the role of a cultural broker provides an important link to building sustainable and collaborative global health partnerships (Underwood et al., 2016). Lastly, although it may appear obvious, those who occupy the critical role of a cultural broker must be remunerated appropriately, at rates which reflect the remuneration of individuals in similar roles at the sending institutions.

\subsubsection{Research}

To date, there is a scarcity of empirical literature dedicated to exploring the ethical complexities and implications of GHPs in nursing. While this study's findings contribute a unique contribution to our understanding of nursing students' experiences of GHPs, and in particular, some of the more challenging aspects of GHPs, a number of avenues for future research warrant attention.

Findings from this study indicate that the way in which nursing students processed their moral uncertainty resulted, at times, in the perpetuation of hurtful and damaging stereotypes of the host communities. Further, exploration of students' experiences of moral uncertainty identified times when the care of local healthcare recipients was - or had the potential to be jeopardized. Such findings are not limited to the results of this study (Martin et al., 2013; Abedini et al., 2012); nevertheless, the seriousness of these claims warrant further investigation, particularly as it relates to host partners' perceptions of the benefits and risks of GHPs and to trainees' experiences of practicing beyond one's scope of practice in the international context. However, due to the sensitive nature of this topic and to the inherent differences that exist 
between standards of care globally, explorations of this topic should be facilitated - to the greatest extent possible - in a no-fault manner.

As discussed in greater depth in my second chapter, research within this topic area has largely originated from the field of medicine. Existing studies have explored perspectives of host medical students (Elobu et al., 2014), host healthcare professionals (Bozinoff et al., 2014; Kraeker \& Chandler, 2013; Kumwenda et al., 2015; Lukolyo et al., 2016), and recipients of the care provided by GHP participants (DeCamp et al., 2014). More recently, however, a qualitative exploration of host partners' perceptions of GHPs provided important insight on the topic from a nursing perspective (Underwood et al., 2016). Additionally, important work has been done to incorporate host partners' perspectives into competencies for global health education (see: Cherniak et al., 2017). However, despite this positive shift toward exploring components of international practicums, such as mutuality and reciprocity, future research to further investigate the more troubling aspects of GHPs, is needed. Of particular interest would be both qualitative and quantitative investigations of the hosts' perceptions of the ethical challenges of GHPs in nursing. This could be facilitated through an exploration of some of more challenging or uncomfortable elements of hosting international nursing students. However, due to the sensitive nature of this topic, and in acknowledgment of the power differentials engrained between highincome and low- and middle-income countries, this research would need to be approached through research methods that include both observational and interview methods so that the burden placed on participants to disclose the more challenging elements of GHPs is reduced.

\subsection{Limitations}

Despite the important contributions that this study makes to the literature on undergraduate nursing students' experiences of moral uncertainty during GHPs, several limitations inherent in 
this study exist. Firstly, participants were recruited to participate in this study via convenience sampling. While benefits of convenience sampling do exist (Kriska, Sass, \& Fulcomer, 2013; Laerd, 2012), limitations associated with this type of non-probability sampling must be acknowledged. Specifically, findings must be interpreted cautiously as the sample that was selected to participate in this study may not be representative of the population of interest as a whole (Laerd, 2012). For instance, one must reflect on the reasons behind why some participants elected to take part in this study, while others did not (Laerd, 2012). In the case of our research, nursing students who encountered more ethically and/or morally challenging GHP experiences may have been more likely to participate in our research, than students who did not. Furthermore, despite sampling from cohorts of undergraduate nursing students who participated in a GHP between the years of 2011 and 2016, inclusive, no participants from the 2015 cohort expressed an interest in participating in this study. As such, while this study provides important insight into the more challenging aspects of GHPs, it may not represent the breadth of experiences that students encounter during overseas practicums.

Secondly, in light of the sampling method used, the demographics of our sample represent a largely homogenous population: the mean age of participants was 26.5 years, with 15 of the 17 participants self-identifying as female, and the majority of participants self-identifying as either ‘Caucasian’, ‘white’, or ‘European’. Furthermore, participants were recruited from a single university in Western Canada, who had participated in a GHP in either West Africa (Ghana) or sub-Saharan Africa (Zambia). Thus, while this study offers robust insight into the moral uncertainty that nursing students from UBC’s Okanagan campus experienced during GHPs, these finding can not be transferred to all nursing programs that offer global health experiences. To further emphasize this limitation, this study’s findings indicate that much of the moral 
uncertainty that nursing students experienced during GHPs was related to the inadequacy of training in critical pedagogies, both prior to, and during, GHPs. Thus, given the diversity of educational instruction across programs which offer GHPs in nursing, caution must be taken when interpreting and attempting to transfer the results of this study.

\subsection{Conclusions}

Findings from this study indicate that nursing students from one university in Canada experienced a considerable amount of moral uncertainty during their GHP experience. Underlying much of the moral uncertainty was a misalignment between what participants expected their global health experience to entail, and what was experienced in reality. Participants reflected deeply on these disparities, evidencing the development of a critical approach to global citizenship, as well as a heightened social consciousness. However, further integration of critical and transformative pedagogies into pre-departure training for GHPs, specifically, and undergraduate nursing education, more broadly, is needed. 


\section{Works Cited}

Abedini, N.C., Gruppen, L.D., Kolars, J.C., \& Kumagai, A.K. (2012). Understanding the effects of short-term international service-learning trips on medical students. Academic Medicine, 87(6), 820-828. doi: 10.1097/AMC.0b013e31825396d8

Adams, E. (2010). The joys and challenges of semi-structured interviewing. Community Practitioner, 83(7), 18-21.

Adams, L., Wagner, C., Nutt, C., \& Binagwaho, A. (2016). The future of global health education: Training for equity in global health. BMC Medical Education, 16(1), 1-7. doi:10.1186/s12909-016-0820-0

Afriyie Asenso, B., Reimer-Kirkham, S., \& Astle, B. (2013). In real time: Exploring nursing students' learning during an international experience. International Journal of Nursing Education Scholarship, 10(1), 227-236. doi: 10.1515/ijnes-2012-0045

Anderson, J. M., \& McCann, E. K. (2002). Toward a post-colonial feminist methodology in nursing research: Exploring the convergence of post-colonial and black feminist scholarship. Nurse Researcher, 9(3), 7.

Anderson, K.C., Slatnik, M.A., Pereira, I., Cheung, E., Xu, K., \& Brewer, T.F. (2012). Are we there yet? Preparing Canadian medical students for global health electives. Global Health Education, 87(2), 206-209. doi: 10.1097/ACM.0b013e31823e23d4

Andreotti, V. (2006). Soft versus critical global citizenship education. Policy \& Practice - A Development Education Review, 3, 40-51. Retrieved from: https://www.developmenteducationreview.com

Asao, S., Lewis, B., Harrison, J.D., Glass, M., \& Penick Brock, T. (2017). Ethics simulation in global health training (ESIGHT). MedEdPORTAL, 13, 1-10. doi:10.15766/mep_23748265.10590

Battat, R., Seidman, G., Chadi, N., Chanda, M., Nehme, J., Hulme, J., . . Brewer, T. (2010). Global health competencies and approaches in medical education: A literature review. BMC Medical Education, 10(1), 94-94. doi:10.1186/1472-6920-10-94

Bentley, R., \& Ellison, K. J. (2007). Increasing cultural competence in nursing through international service-learning experiences. Nursing Educator, 32, 207-211.

Beumer, C. M. (2008). Innovative solutions: The effect of a workshop on reducing the experience of moral distress in an intensive care unit setting. Dimensions of Critical Care Nursing, 27(6), 263-267. doi:10.1097/01.DCC.0000338871.77658.03

Bessette, J., \& Camden, C. (2017). Pre-departure training for student global health experiences: A scoping review. Physiotherapy Canada, 69(4), 343-350. doi: 10.3138/ptc.2015-86GH 
Bohman, D. M., \& Borglin, G. (2014). Student exchange for nursing students: Does it raise cultural awareness'? A descriptive, qualitative study. Nurse Education in Practice, 14(3), 259-264.

Bozinoff, N., Dorman, K.P., Kerr, D. Roebbelen, E., Rogers, E., Hunter, A...Kraeker, C. (2014). Toward reciprocity: Host supervisor perspectives on international medical electives. Medical Education, 48(4), 397-404. doi:10.1111/medu.12386

Brown, H., Rodney, P., Pauly, B., Varcoe, C., \& Smye, V. (2004). Working within the landscape: Nursing ethics. In: Storch, J., Rodney, P., \& Starzomski, R. (Eds.), Toward a moral horizon: Nursing ethics for leadership and practice (pp. 126-153). Toronto, ON: Pearson.

Burgess, C.A., Reimer Kirkham, S., \& Astle, B. (2014). Motivations and international clinical placements: Shifting nursing students to a global citizenship perspective. International Journal of Nursing Education Scholarship, 11(1), 75-82. doi: 10.1515/ijnes-2013-0056

Burston, A. S., \& Tuckett, A. G. (2013). Moral distress in nursing: Contributing factors, outcomes and interventions. London, England: SAGE Publications. doi:10.1177/0969733012462049

Canadian Coalition for Global Health Research (CCGHR). (2015). CCGHR Principles for Global Health Research [Online]. Ottawa, Canada: CCGHR. Available at: http://www.ccghr.ca/resources/principles-global-health-research/ [Accessed 04 April 2018].

Canadian Nurses’ Association. (2008). Code of ethics for registered nurses (2008 centennial Ed.). Ottawa: The Association.

Canadian Nurses’ Association. (2017). Code of ethics for registered nurses [Online]. Ottawa, Canada: The Association. Available at: https://www.cna-aiic.ca/html/en/Code-of-Ethics2017-Edition/files/assets/basic-html/page-1.html\# [Accessed 04 April 2018].

Caldwell, P., \& Purtzer, M. A. (2014). Long-term learning in a short-term study abroad program: "Are we really truly helping the community?” Public Health Nursing, 32(5), 577-583.

Callahan, D. (1999). The social science and the task of bioethics. Daedalus, 128(4), 275-294. Retrieved from http://www.jstor.org/stable/20027596

Cantrell, M.A., Browne, A.M., \& Lupinacci, P. (2005). The impact of a nurse externship program on the transition process from graduate to registered nurse: Part 1 quantitative findings. Journal for Nurses in Staff Development, 21, 187-195.

Carpenter, L. J., \& Garcia, A. A. (2012). Assessing outcomes of a study abroad course for nursing students. Nursing Education Perspectives, 33(2), 85-89.

Castree, N., Kitchin, R., \& Rogers, A. (2013). Global North. In A Dictionary of Human Geography. Retrieved from www.oxfordreference.com 
Cherniak, W., Latham, E., Astle, B., Anguyo, G., Beaunoir, T., Buenaventura, J.H, ... Evert, J. (2017). Host perspectives on short-term experiences in global health: A survey. Lancet Global Health, 5, S9-S9. doi: 10.1016/S2214-109X(17)30116-X

Cleaver, S., Magalhaes, L., Bond, V., Polatajko, H., \& Nixon, S. (2015). Research principles and research experiences: critical reflection on conducting a $\mathrm{PhD}$ dissertation on global health and disability. Disability and the Global South, 3(2), 1022-1043.

Cohen, J.S., \& Erickson, J.M. (2006). Ethical dilemmas and moral distress in oncology nursing practice. Clinical Journal of Oncology Nursing, 10(6), 775-782. doi: 10.1188/06.CJON.775-780

College of Registered Nurses of British Columbia (CRNBC). 2013. Professional Standards for Registered Nurses and Nurse Practitioners [Online]. Vancouver, Canada: CRNBC. Available at https://www.crnbc.ca/Standards/Lists/StandardResources/128ProfessionalStandards.pdf

College of Registered Nurses of British Columbia (CRNBC). (2017, March 31). CRNBC commits to a safer health system for First Nations and Aboriginal People. Available at https://www.crnbc.ca/crnbc/Announcements/2017/Pages/Declaration_Cultural_Safety_H umility.aspx

Consortium of Universities for Global Health (CUGH). (2017). Global Health Education Competencies Toolkit. Available at https://www.cugh.org/sites/default/files/CUGH_GHE_Competency_Tool-kit_2017_0.pdf

Creswell, J. (2007). Qualitative Inquiry \& Research Design: Choosing Among Five Approaches ( $2^{\text {nd }} E d$.). New Delhi, India: Sage Publications, Inc.

Crump, J.A., \& Sugarman, J. (2010). Ethics and best practice guidelines for training experiences in global health. American Journal of Tropical Medicine and Hygiene, 83(6), 1178-1182.

Crump, J.A., \& Sugarman, J. (2008). Ethical considerations for short-term experiences by trainees in global health. Jama, 300(12), 1456-1458. doi:10.1001/jama.300.12.1456

Dawson, A. (2011). Public health ethics: Key concepts and issues in policy and practice. Cambridge: Cambridge University Press.

DeCamp, M., Enumah, S., O’Neill, D., \& Sugarman, J. (2014). Perceptions of a short-term medical programme in the Dominican Republic: Voices of care recipients. Global Public Health, 9(4), 411-425. doi:10.1080/17441692.2014.893368

DeCamp, M., Rodriguez, J., Hecht, S., Barry, M., \& Sugarman, J. (2013). An ethics curriculum for short-term global health trainees. Globalization and Health, 9(5), 1-10.

Dell, E.M., Varpio, L., Petrosoniak, A., Garjaria, A., \& McCarthy, A.E. The ethics and safety of medical student global health electives. (2014). International Journal of Medical Education, 5, 63-72. 
Denzin, N.K. (1978). The research act: A theoretical introduction to sociological methods $\left(2^{\text {nd }}\right.$ Ed.). New York: McGraw-Hill.

Denzin, N. K., Lincoln, Y. S., \& Xwi7xwa Collection. (2011). The sage handbook of qualitative research (4th ed.). Thousand Oaks, Calif: Sage

Dobson, A. (2006). Thick cosmopolitanism. Political Studies, 54(1), 165-184. doi:10.1111/j.1467-9248.2006.00571.x

Dowell, J., \& Merrylees, N. (2009). Electives: Isn't it time for a change? Medical Education, 43(2), 121-126.

Dunn, M., Sheehan, M., Hope, T., \& Parker, M. (2012). Toward methodological innovation in empirical ethics research. Cambridge Quarterly of Healthcare Ethics, 21, 466-480. doi: 10.1017/S0963180112000242

Elit, L., Hunt, M., Redwood-Campbell, L., Ranford, J., Adelson, N., \& Schwartz, L. (2011). Ethical issues encountered by medical students during international health electives. Medical Education, 45(7), 704-711.

Elobu, A.E., Kintu, A., Galukande, M., Kaggwa, S., Mijjumbi, C., Tindiwebwa, J...Lipnick, M. (2014). Evaluating international global health collaborations: Perspectives from surgery and anesthesia trainees in Uganda. (2014). Surgery, 155(4), 585-592. doi: http://dx.doi.org/10.1016/j.surg.2013.11.007

Epstein, E., \& Hamric, A. (2009). Moral distress, moral residue, and the crescendo effect. Journal of Clinical Ethics, 20(4), 330-342.

Ericksen, J., Rodney, P., \& Starzomski, R. (1995). When is it right to die? The Canadian Nurse, 91(8), 29.

Erlen, J.A., \& Frost, B. (1991). Nurses’ perceptions of powerlessness in influencing ethical decisions. Western Journal of Nursing Research, 13(3), 397-407. doi:10.1177/019394599101300309

Evanson, T., \& Zust, B.L. (2006). "Bittersweet knowledge": The long-term effects of an international experience. Journal of Nursing Education, 45(10), 412-419.

Forde, R. (2012). How can empirical ethics improve medical practice? Cambridge Quarterly of Healthcare Ethics, 21, 517-526. doi: 10.1017/S096318011200028X

Frith, L. (2010). Symbiotic empirical ethics: A practical methodology. Bioethics, 26, 198-206. doi: 10.1111/j.1467-8519.2010.01843.x

Galvin, J., Suominen, E., Morgan, C., O’Connell, E.J., \& Smith, A.P. (2015). Mental health nursing students' experiences of stress during training: A thematic analysis of qualitative interviews. Journal of Psychiatric and Mental Health Nursing, 22, 773-783. 
Giddings, L.S. (2005). A theoretical model of social consciousness. Advances in Nursing Science, 28(3), 224-239.

Gower, S., Duggan, R., Dantas, J.A.R., \& Boldy, D. (2016). Motivations and expectations of undergraduate nursing students undertaking international clinical placements. Journal of Nursing Education, 55(9), 487-494. doi: 10.3928/01484834-2060816-02

Hamric, A.B. (2000). Moral distress in everyday ethics. Nursing Outlook, 48, 199-201.

Hanna, D. R. (2005). The lived experience of moral distress: Nurses who assisted with elective abortions. Research and Theory for Nursing Practice, 19(1), 95.

Hanson, L., Harms, S., \& Plamondon, K. (2011). Undergraduate International Medical Electives: Some ethical and pedagogical considerations. Journal of Studies in International Education, 15: 171-185.

Harrison, J.D., Logar, T., Le, P., \& Glass, M. (2016). What are the ethical issues facing globalhealth trainees working overseas? A multi-professional qualitative study. Healthcare, 4(3), 1-9. doi: 10.3390/healtcare4030043.

Hoe Harwood, C., Reimer-Kirkham, S., Sawatzky, R., Terblanche, L., \& Van Hofwegen, L. (2009). Innovation in community clinical placements: A canadian survey. International Journal of Nursing Education Scholarship, 6(1), 28. doi:10.2202/1548-923X.1860

Hsieh, H-F., \& Shannon, S.E. (2005). Three approaches to qualitative content analysis. Qualitative Health Research, 15(9), 1277-1288. doi: 10.1177/1049732305276687

Huffman, D. M., \& Rittenmeyer, L. (2012). How professional nurses working in hospital environments experience moral distress: A systematic review. Critical Care Nursing Clinics of North America, 24(1), 91-100. doi:10.1016/j.ccell.2012.01.004

Izadnegahdar, R., Correia, S., Ohata, B., Kittler, A., Kuile, S., Vaillancourt, S...Brewer, T.F. (2008). Global health in Canadian medical education: Current practices and opportunities. Academic Medicine, 83, 192-198.

Jameton, A. (1984). Nursing practice: The ethical issues. Englewood Cliff, New Jersey: Prentice Hall.

Kälvemark, S., Höglund, A. T., Hansson, M. G., Westerholm, P., \& Arnetz, B. (2004). Living with conflicts-ethical dilemmas and moral distress in the health care system. Social Science \& Medicine, 58(6), 1075-1084. doi:10.1016/S0277-9536(03)00279-X

Kelleher, S., FitzGerald, S., \& Hegarty, J. (2016). Factors that influence nursing and midwifery students' intentions to study abroad: A qualitative study used the theory of planned behaviour. Nurse Education Today, 44, 157-164. doi:

http://dx.doi.org/10.1016/j.nedt.2016.05.019

Kelly, B. (1998). Preserving moral integrity: A follow-up study with new graduate nurses. Journal of Advanced Nursing, 28(5), 1134-1145.

Kent-Wilkinson, A., Dietrich Leurer, M., Luimes, J., Ferguson, L., \& Murray, L. (2015). Studying abroad: Exploring factors influencing nursing students' decisions to apply for 
clinical placements in international settings. Nurse Education Today, 35, 941-947. doi: http://dx.doi.org/10.1016/j.nedt.2015.03.012

Kollar, S. J., \& Ailinger, R. L. (2002). International clinical experiences: Long-term impact on students. Nurse Educator, 27(1), 28-31.

Koplan, J.P., Bond, T.C., Merson, M.H., Reddy, K.S., Rodriguez, M.H., Sewankambo, N.K., \& Wasserheit, J.N. (2009). Towards a common definition of global health. Lancet, 373(9679). 1993-1995. doi: http://dx.doi.org/10.1016/S0140-6736(09)60332-9

Kraeker, C., \& Chandler, C. (2013). "We learn from them, they learn from us": Global health experiences and host perceptions of visiting health care professionals. Academic Medicine, 88(4), 483-487.

Kriska, S.D., Sass, M.M., \& Fulcomer, M.C. (2013). Assessing limitations and uses of convenience samples: A guide for graduate students. JSM: Section on Statistical Education, 2828-2834. Retrieved from http://www.statlit.org/pdf/2013-Kriska-ASA.pdf

Kumwenda, B., Dowell, J., Daniels, K., \& Merrrylees, N. (2015). Medical electives in subSaharan Africa: A host perspective. Medical Education, 49(6), 623-633. doi: 10.1111/medu.12727

Kumwenda, B., Royan, B., Ringsell, P., \& Dowell, J. (2014). Western medical students' experiences on clinical electives in sub-Saharan Africa. Medical Education, 48(6), 593603. doi: 10.1111/medu.12477

Kvale, S. (2008). Interviews: Learning the craft of qualitative research interviewing ( ${ }^{\text {rd }}$ Ed.). London: Sage.

Laerd. (2012). Convenience sampling. Retrieved from http://dissertation.laerd.com/conveniencesampling.php

Lahey, T. (2012). A proposed medical school curriculum to help students recognize and resolve ethical issues of global health outreach work. Academic Medicine, 87, 210-215. doi: 10.1097/ACM.0b013e31823f3fb1

Larson, K. L., Ott, M., \& Miles, J. M. (2010). International cultural immersion: En vivo reflections in cultural competence. Journal of Cultural Diversity, 17(2), 44-50.

Leffers, J., \& Mitchell, E. (2011). Conceptual model for partnership and sustainability in global health. Public Health Nursing, 28(1), 91-102. doi:10.1111/j.1525-1446.2010.00892.x

Leininger, M. (1998). Nursing education exchanges: concerns and benefits. Journal of Transcultural Nursing, 9(2), 57-63.

Levi, A. (2009). The ethics of nursing student international clinical experiences. Journal of Obstetric, Gynecologic, and Neonatal Nursing, 38(1), 94-99. doi: 10.1111/j.15526909.2008.00314.x 
Levine, M. A., \& Perpetua, E. M. (2006). International immersion programs in baccalaureate nursing education: Professor and student perspectives. Journal of Cultural Diversity, 13(1), 20-26.

Lincoln, Y. S., \& Guba, E. G. (1985). Naturalistic inquiry. Beverly Hills, Calif: Sage Publications.

Logar, T., Le, P., Harrison, J. D., \& Glass, M. (2015). Teaching corner: "First do no harm": Teaching global health ethics to medical trainees through experiential learning. Journal of Bioethical Inquiry, 12(1), 69-78. doi:10.1007/s11673-014-9603-7

Lukolyo, H., Rees, C. A., Keating, E. M., Swamy, P., Schutze, G. E., Marton, S., \& Turner, T. L. (2015;2016;). Perceptions and expectations of host country preceptors of short-term learners at four clinical sites in sub-Saharan Africa. Academic Pediatrics, 16(4), 387-393. doi:10.1016/j.acap.2015.11.002

Lützén, K., Kvist, B. E. (2012). Moral distress: A comparative analysis of theoretical understandings and inter-related concepts. HEC Forum, 24(1), 13-25. doi:10.1007/s10730-012-9178-9

Malloy, D. C., Hadjistavropoulos, T., McCarthy, E. F., Evans, R. J., Zakus, D. H., Park, I.,...Williams, J. (2009). Culture and organizational climate: Nurses' insights into their relationship with physicians. Nursing Ethics, 16(6), 719-733. doi:10.1177/0969733009342636

Martin, B.M., Love, T.P., Srinivasan, J., Sharma, J., Pettitt, B., Sullivan, C...Brewster, L.P. (2013). Designing an ethics curriculum to support global health experiences in surgery. Journal of Surgical Research, 187(2), 367-370.

McCall, D., \& Iltis, A. S. (2014). Health care voluntourism: Addressing ethical concerns of undergraduate student participation in global health volunteer work. HEC Forum, 26(4), 285-297. doi: 10.1007/s10730-014-9243-7

McCarthy, A. E., Petrosoniak, A., \& Varpio, L. (2013). The complex relationships involved in global health: A qualitative description. BMC Medical Education, 13, 136-144.

Meaney, M. (2002). Taking action against moral distress. Mosby, Inc. doi:10.1067/mcm.2002.127993

Megel, M.E., Nelson, A.E., Black, J.,Vogel, J., \& Uphoff, M. (2013). A comparison of student and faculty perception of clinical post-conference learning environment. Nurse Education Today, 33, 525-529.

Meltzer, L., \& Huckaboy, L. (2004). Critical care nurses' perceptions of futile care and its effect on burnout. American Journal of Critical Care, 13(3), 202-208

Mill, J., Astle, B., Ogilvie, L., \& Gastaldo, D. (2010). Linking global citizenship, undergraduate nursing education, and professional nursing curricular innovation in the 21st century. Advances in Nursing Science, 33(3), E1-E11. doi:10.1097/ANS.0b013e3181eb416f 
Mill, J.E., Yonge, O.J., \& Cameron, B.L. (2005). Challenges and opportunities of international clinical practica. International Journal of Nursing Education Scholarship, 2(1), 1-13.

Morse, J.M., \& Field, P.A. (1995). Qualitative Research Methods for Health Professionals $\left(2^{\text {nd }}\right.$ Ed.). Thousand Oaks, CA: Sage.

Oberle, K. \& Raffin, S. (2009). Ethics in Canadian nursing practice: Navigating the journey. Toronto: Pearson Prentice Hall.

Ohnishi, K., Ohgushi, Y., Nakano, M., Fujii, H., Tanaka, H., Kitaoka, K.,...Narita, Y. (2010). Moral distress experienced by psychiatric nurses in japan. Nursing Ethics, 17(6), 726740. doi:10.1177/0969733010379178

Pauly, B. M., Varcoe, C., \& Storch, J. (2012). Framing the issues: Moral distress in health care. HEC Forum, 24(1), 1-11. doi:10.1007/s10730-012-9176-y

Patton, M. (1990). Qualitative evaluation and research methods. Beverly Hills, CA: Sage.

Pendry, P. (2007). Moral distress: Recognizing it to retain nurses. Nursing Economics, 25(4), 217-221.

Petrosoniak, A., McCarthy, A., \& Varpio, L. (2010). International health electives: Thematic results of student and professional interviews. Medical Education, 44(7), 683-689.

Pinto, A.D., \& Upshur, R.E. (2009). Global health ethics for students. Developing World Bioethics, 9(1), 1-10. doi: 10.1111/j.1471-8847.2007.00209.x.

Pogge, T. (2001). Priorities of global justice. Metaphilosophy, 32(1/2), 6-24. doi:10.1111/14679973.00172

Potgeiter, E. (2012). Clinical teaching: Developing critical thinking in student nurses. Professional Nurse Today, 16(2), 4-8.

QSR International Pty Ltd. (2017). Nvivo qualitative data analysis Software: Version 11, 2017.

Racine, L., \& Perron, A. (2012). Unmasking the predicament of cultural voyeurism: A postcolonial analysis of international nursing placements. Nursing Inquiry, 19(3), 190201. doi: 10.1111/j.1440-1800.2011.00555.x

Radzvin, L. C. (2011). Moral distress in certified registered nurse anesthetists: Implications for nursing practice. AANA Journal, 79(1), 39.

Reimer Kirkham, S., Van Hofwegen, L., \& Pankratz, D. (2009). Keeping the vision: Sustaining social consciousness with nursing students following international learning experiences. International Journal of Nursing Education Scholarship, 6(1), 1-15. doi: 10.2202/1548923X.1635

Rice, E. M., Rady, M. Y., Hamrick, A., Verheijde, J. L., \& Pendergast, D. K. (2008). Determinants of moral distress in medical and surgical nurses at an adult acute tertiary 
care hospital. Journal of Nursing Management, 16(3), 360-373. doi:10.1111/j.13652834.2007.00798.x

Richards, L., \& Morse, J.M. (2013). Readme first for a user's guide to qualitative methods (3 ${ }^{\text {rd }}$ Ed.). Thousand Oaks, CA: Sage.

Rodney, P.A. (2017). What we know about moral distress. American Journal of Nursing, 117(2), S7-S10.

Rodney, P., Brown, H., \& Liaschenko, J. (2004). Moral agency: Relational connects and trust. In: Storch, J., Rodney, P., \& Starzomski, R. (Eds.), Toward a moral horizon: Nursing ethics for leadership and practice (pp. 154-177). Toronto, ON: Pearson.

Rodney, P., Pauly, B., \& Burgess, M. (2004). Our theoretical landscape: Complementary approaches to health care ethics. In: Storch, J., Rodney, P., \& Starzomski, R. (Eds.), Toward a moral horizon: Nursing ethics for leadership and practice (pp. 77-97). Toronto, ON: Pearson.

Rodney, P., \& Starzomski, R. (1993). Constraints on the moral agency of nurses. The Canadian Nurse, 89(9), 23.

Rodney, P., \& Varcoe, C. (2001). Towards ethical inquiry in the economic evaluation of nursing practice. The Canadian Journal of Nursing Research, 33(1), 35.

Ruddock, H. C., \& Turner, d. S. (2007). Developing cultural sensitivity: Nursing students' experiences of a study abroad programme. Journal of Advanced Nursing, 59(4), 361-369.

Rushton, C. H. (1992). Care-giver suffering in critical care nursing. Heart \& Lung: The Journal of Critical Care, 21(3), 303.

Rushton, C.H. (2017). Cultivating moral resilience. American Journal of Nursing, 117(2), S11S15.

Rushton, C., Caldwell, M., \& Kurtz, M. (2016). Moral distress: A catalyst in building moral resilience. American Journal of Nursing, 116(7), 40-49.

Ryan-Krause, P. (2016). Short-term global experiences: Reflections, themes, and implications. Journal of Nursing Education, 55(7), 379-384. doi:10.3928/0148483420160615-04

Sandelowski, M. (1993). Rigor or rigor mortis: The problem of rigor in qualitative research revisited. Advances in Nursing Science, 16(2), 1-8.

Sandelowski, M. (2000). Whatever happened to qualitative description? Research in Nursing \& Health, 23, 334-340.

Sandeloswki, M., \& Barroso, J. (2003). Classifying the findings in qualitative studies. Qualitative Health Research, 13(7), 905-923. doi: 10.1177/1049732303253488

Sandelowski, M. (2009). What's in a name? Qualitative description revisited. Research in Nursing \& Health, 33, 77-84. doi: 10.1002/nur.20362 
Sedgwick, P. (2013). Convenience Sampling. BMJ, 347(oct25 2), f6304-f6304. doi: 10.1136/bmj.f6304

Seymour, B., Benzian, H., \& Kalenderian, E. (2012). Voluntourism and global health: Preparing dental students for responsible engagement in international programs. Journal of Dental Education, 77(10), 1252-1257.

Sherwin, S. (1992). No longer patient: Feminist ethics and healthcare. Philadelphia: Temple University Press.

Sloand, E., Bower, K., \& Groves, S. (2008). Challenges and benefits of international clinical placements in public health nursing. Nurse Educator, 33(1), 35-38.

Shah, S., \& Wu, T. (2008). The medical student global health experience: Professionalism and ethical implications. Journal of Medical Ethics, 34(5), 375-378. doi: 10.1136/jme.2006.019265

Snyder, J., Dharamsi, S., \& Crooks, V.A. (2011). Fly-by medical care: Conceptualizing the global and local social responsibilities of medical tourists and physician volunteers. Globalization Health, 7(6), 1-14.

Spivak, G. (2003). A conversation with Gayatri Chakravorty Spivak: Politics and the imagination, interview by J Sharpe. Signs: Journal of Women in Culture and Society, 28609-624.

Storch, J. L., Peter, E., \& Canadian Nurses Association. (2008). The CNA code of ethics for registered nurses. Nursing Leadership, 21(2), 28-33. doi:10.12927/cjnl.2008 .19872

Thorne, S. (2000). Data analysis in qualitative research. Evidence Based Nursing, 3(3), 68-70. doi:10.1136/ebn.3.3.68

Thorne, S. (2008). Interpretive description. Walnut Creek, CA: Left Coast Press.

Thorne, S. (2000). Data analysis in qualitative research. Evidence-Based Nursing, 3(3), 68-70. doi:10.1136/ebn.3.3.68

Thorne, S., Kirkham, S. R., \& MacDonald-Emes, J. (1997). Interpretive description: A noncategorical qualitative alternative for developing nursing knowledge. Research in Nursing \& Health, 20(2), 169-177. doi:10.1002/(SICI)1098240X(199704)20:2<169::AID-NUR9>3.3.CO;2-B

Thorne, S., Kirkham, S. R., \& O'Flynn-Magee, K. (2004). The analytic challenge in interpretive description. International Journal of Qualitative Methods, 3(1), 1-11. doi:10.1177/160940690400300101 
Truth and Reconciliation Commission of Canada. (2015). Truth and reconciliation commission of Canada: Call to action. Retrieved from http://nctr.ca/assets/reports/Calls_to_Action_English2.pdf

Tufford, L., \& Newman, P. (2010). Bracketing in qualitative research. Qualitative Social Work, 11(1), 80-96. doi: 10.1177/1473325010368316

Underwood, M., Gleeson, J., Konnert, C., Wong, K., \& Valerio, B. (2016). Global host partner perspectives: Utilizing a conceptual model to strengthen collaboration with host partners for international nursing student placements. Public Health Nursing, 33(4), 351-359. doi: 10.1111/phn.12258

United Nations. (2017). Progress towards the Sustainable Development Goals: Report of the Secretary-General. Retrieved from http://www.un.org/ga/search/view_doc.asp?symbol=E/2017/66\&Lang=E

United Nations Development Programme. (2007). South-South Cooperation. Retrieved from http://ssc.undp.org/content/dam/ssc/documents/exhibition_triangular/SSC ExPoster1.pdf

University of British Columbia. (2011). The Ethics of International Engagement and ServiceLearning [Online]. Vancouver, Canada: UBC Go Global. Available at: http://ethicsofisl.ubc.ca/

University of British Columbia. (2015). Scope of Practice: Psychomotor Skills for BSN Students [Online]. Kelowna, Canada: School of Nursing, Okanagan Campus. Available at: https://nursing.ok.ubc.ca/wp-content/uploads/sites/6/2015/07/BSN-Scope-of-Practicepassed-24-Aug-2015.pdf

University of British Columbia. (2017). Global Health Practicum. Kelowna, Canada: Go Global, UBC Okanagan Campus. Available at: http://students.ok.ubc.ca/global/outgoing/gsp/current-programs/2016nursing.html

Wallace, L.J., \& Webb, A. (2014). Pre-departure training and the social accountability of international medical electives. Education for Health, 27(2), 143-147. doi: 10.4103/13576283.143745

Webster, G.C., \& Baylis, F.E. (2000). Moral Residue. In: Rub, S.B. and Zoloth, L. (Eds.), Margin of error: The ethics of mistakes in the practice of medicine (pp. 217-230). Hagerstown, MD: University Publishing Group.

Willis, G. (1999). Cognitive interviewing: A “how to” guide. Research Triangle Institute. Retrieved from http://appliedresearch.cancer.gov/archive/cognitive/interview.pdf

World Health Organization. (2015). Health in 2015: From millennium development goals to sustainable development goals. Available at http://apps.who.int/iris/bitstream/handle/10665/200009/9789241565110_eng.pdf;jsession id=4352EFEAFA5B29F4D45ACB8D619F7275? sequence $=1$ 
Vance, C.M., Vaiman, T., \& Anderson, V. (2009). The vital liaison role of host country nationals in MNC knowledge management. Human Resource Management, 48(4), 649-659. doi: 10.1002/hrm.20307

Varcoe, C. (2004). Widening the scope of ethical theory, practice, and policy: Violence against women as an illustration. In: Storch, J., Rodney, P., \& Starzomski, R. (Eds.), Toward a moral horizon: Nursing ethics for leadership and practice (pp. 414-432). Toronto, ON: Pearson.

Yeo, M. \& Ford, A. (1996). In M. Yeo and A. Moorhouse (Eds.), Concepts and cases in nursing ethics, $2^{\text {nd }}$ ed. (pp. 267-306). Peterborough, ON: Broadview.

Zaidi, M.Y., Haddad, L., \& Lathrop, E. (2015). Global health opportunities in obstetrics and gynecology training: Examining engagement through an ethical lens. American Journal of Tropical Medicine and Hygiene, 93(6), 1194-1200 


\section{Appendices}

\section{Appendix A: Search Strategy}

Database: Ovid MEDLINE

1 International Educational Exchange

2 study abroad.tw.

3 ((international\$ or foreign) adj2 (partner\$ or program\$ or placement\$ or experienc\$)).tw.

4 (global health adj2 (program\$ or experience\$ or education\$)).tw.

5 (world health adj2 (program\$ or experience\$ or education\$)).tw.

6 or $1-5$

7 ethic\$.tw.

8 exp ethics/

9 or $/ 7-8$

$10 \quad 6$ and 9

11 limit 10 to (abstracts and English language) 


\section{Appendix B: PRISMA Flow Diagram}

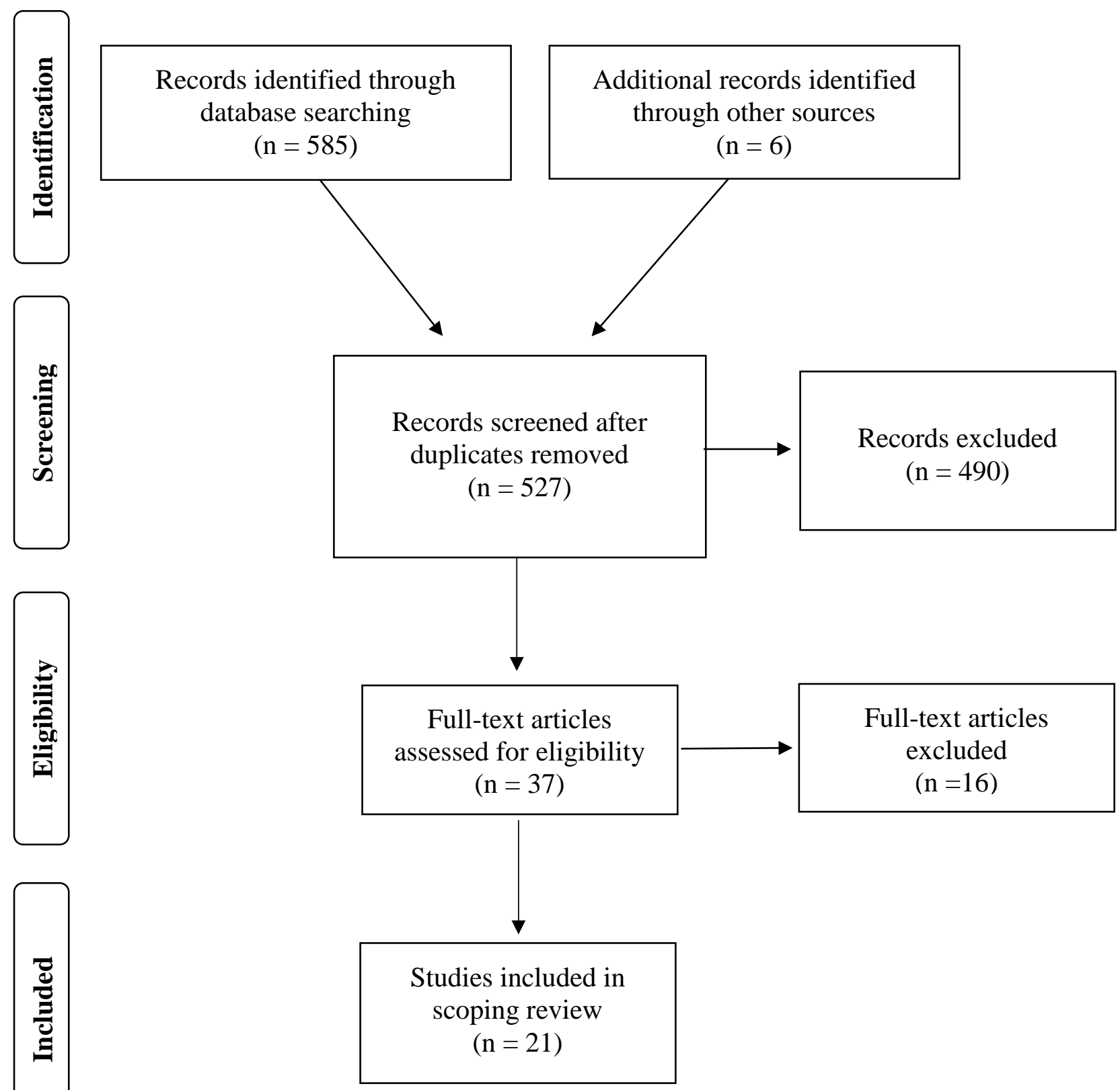




\section{Appendix C: Recruitment Bulletin \\ EMAIL CORRESPONDENCE}

Date

Dear Graduates,

On behalf of Graduate Nursing Student, Madeleine Greig, and the research team we are inviting your participation in a research study entitled, "The Ethical Implications of Global Health Practicums in Nursing”. The aim of this study is to gain a better understanding of the ethical implications of global health practicums in the context of undergraduate nursing education.

Our records indicate you participated in a Global Health experience in your fourth year of the nursing program. Therefore, we are requesting your participation in this study. Please see the attached information sheet provided by the research team for more information on the research study, and ways that you can become involved.

Sheila Epp RN MN, Senior Instructor

Associate Director, School of Nursing

Office: Arts 343 | Phone: 250-807-9975

Cell phone: 250-862-7334

Email: sheila.epp@ubc.ca

Faculty of Health and Social Development

ARTS 360 - 1147 Research Rd.

University of British Columbia | Okanagan Campus

Kelowna BC | V1V 1V7 


\section{Appendix D: Consent Form}

THE UN IVER S T Y OF B R I I S H C O L U M I A

\section{Consent Form}

Title of Project: The Ethical Implications of Global Health Practicums in Nursing

Principal Investigator:

Co-Investigator:

Co-Investigator:

Co-Investigator:
Dr. Barbara Pesut, Associate Professor

Health, Ethics and Diversity Lab

University of British Columbia Okanagan

Telephone: 250-807-9955

E-mail: barb.peust@ubc.ca

Madeleine Greig, Graduate Nursing Student

Master of Science in Nursing (MSN) Program

University of British Columbia Okanagan

Telephone: 250-807-8263

E-mail: mbgreig@mail.ubc.ca

Dr. Patricia Marck, Dean

Faculty of Human and Social Development

University of Victoria

E-mail:pmarck@uvic.ca

Dr. Michael Burgess, Chair

Centre for Biomedical Ethics

University of British Columbia

E-mail: michael.burgess@ubc.ca

\section{Background and Purpose of the Study:}

More students than ever are electing to take part in international practicums from health related disciplines. The purpose of this study is to explore the ethical implications of global health practicums attended by undergraduate nursing students from a Canadian university. A better understanding of the types of moral challenges that nursing students experience during global health practicums has the potential to influence the training that student nurses receive before engaging in an overseas nursing experience.

\section{Who is Funding this Study?}

Madeleine Greig, Co-Investigator, is supported by Dr. Barbara Pesut's Canada Research Chair.

\section{Study Procedures:}

Graduates from the undergraduate nursing program at UBC Okanagan who participated in a global health practicum between the years of 2011 and 2016, inclusive, will be asked to participate in a semi-structured interview led by Co-Investigator, Madeleine Greig. Through a variety of questions, both the rewarding and more challenging encounters experienced by nursing students during global health practicums will be explored. In addition, a short survey will 
be completed that collects demographic information, logistics concerning the attended global health practicum, and relevant global health experience. The discussion will be audio-recorded and transcribed for data analysis purposes.

If you are located in the Central, Northern, or Southern regions of the Okanagan Valley, interviews will be conducted in a private meeting room in the Health, Ethics and Diversity Lab at UBC Okanagan, or at a location that is better suited to your needs. If you are located outside of the Central, Northern, or Southern regions of the Okanagan Valley, interviews will be conducted over the phone. The time required for this meeting will range from 30 minutes to 1 hour and refreshments will be served. At the close of the interview Co-Investigator, Madeleine Greig, will ask your permission to participate in a second interview should the need to discuss additional experiences arise. Should this need for a second interview arise, this meeting will again range from 30 minutes to 1 hour of your time.

The results of this study will be made publicly available online via cIRcle, as this research is one component of Co-Investigator, Madeleine Greig’s, Master's thesis.

\section{Risks and Benefits:}

While no explicit risks are expected from participating in this research study, some of the topics that we touch upon may bring up unsettling or upsetting memories. If at any time you feel uncomfortable, vulnerable, or sad, please tell the researcher as we can engage in a conversation around the best way for you to access new or existing psychosocial support systems.

Furthermore, while there are no direct benefits for participating in this study, your participation in this study will help further our understanding of the types of challenges nursing students experience during global health practicums. This information will be used to inform the training nursing students receive prior to going on global health practicums in the future.

\section{Confidentiality:}

Your confidentiality will be respected. Information that discloses who you are will not be released without your consent unless required by law. When you participate in this study you are given a study number and this number is used, rather than your name. The list that matches your name to the study number is kept in a locked filing cabinet and on a computer that is encrypted and protected by a password. Your name, and any information that could identify you, will not be used in any reports of the completed study. We will not reveal any stories that might be linked back to you. Only the Principal Investigator and Co-Investigators listed at the top of the first page of this form, and a transcriptionist hired to transcribe the interview data will be able to access data received from this study. Please note that prior to receiving any interview data, the transcriptionist will have signed a confidentiality agreement. Data from this study will be stored in the Principal Investigator's office.

\section{Questions about the Study?}

If you have any questions or concerns about what we are asking of you, please contact the Principal Investigator or one of the Co-Investigators at any time. Their names and numbers are listed at the top of the first page of this form.

\section{Who do you Contact if you have Complaints or Concerns about the Study?}


If you have any concerns or complaints about your rights as a research participant and/or your experiences while participating in this study, contact any of the following:

- The Research Participant Complaint Line in the UBC Office of Research Ethics toll free at 1-877-822-8598 or the UBC Okanagan Research Services Office at 250-807-8832. It is also possible to contact the Research Complaint Line by e-mail (RSIL@ors.ubc.ca)

\section{Participant Consent and Signature:}

Taking part in this study is entirely voluntary. You have the right to refuse to participate in this study. If you decide to take part, you may choose to pull out of the study at any time without giving a reason for your withdrawal and without any negative impact on your relationship with UBC Additionally, the School of Nursing at UBC Okanagan will not know if you have participated in this research study, or not. If you decide you would like to withdraw from the study, it is up to you whether we can use your data in publications, presentations, and reports about this study.

- Your signature below indicated that you have received a copy of this consent form for you own records.

- Your signature indicates that you consent to participate in this study.

Participant Signature

Date

Printed Name of the Participant Signing Above

I consent to having my interview audio recorded

Yes $\square \quad$ No

Check this box if you would like to receive a summary of the study findings.

E-mail Address (please print):

Mailing Address:

Telephone \# (or where we can leave a message): 


\section{Appendix E: Demographic Survey}

THE U N IVER S I T Y OF B R I T IS H C O L U M B I A

Demographic Form

Title of Project: The Ethical Implications of Global Health Practicums in Nursing

Date:

ID \#

1. In what year were you born?

2. Gender: Male

Female

Prefer not to identify/respond

3. How would you describe your ethnic or cultural identity?

4. What is your highest level of education?

$\square$ University (undergraduate, bachelor's degree)

$\square$ University (graduate, master’s degree, doctorate degree; post-doctorate)

$\square$ Other (specify)

5. What year did you graduate from nursing school?

6. What year did you go on your global health practicum?

7. Where did you go on your global health practicum?

8. Had you travelled or worked in a low or middle-income country prior to going on your global health practicum? Yes
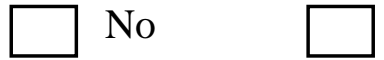

9. Have you travelled or worked in a low or middle-income country since returning home from your global health practicum? Yes No 


\section{Appendix F: Semi-Structured Interview Guide}

\section{Semi-Structured Interview Guide}

\section{Title of Project: The Ethical Implications of Global Health Practicums in Nursing}

Date:

ID \#:

\section{Introduction:}

Firstly, I want to thank you for taking time out of your busy schedule to sit down with me and discuss some of the experiences you had during your global health practicum. Your insight, experiences, and knowledge are so appreciated. If you are feeling comfortable and ready, I will begin by turning on the audio-recording equipment. Remember, you are allowed to pass on any of the questions, stop the interview entirely if you feel uncomfortable, or ask for clarification at any time.

I think the best place for us to begin this conversation will be by getting an over-arching picture of your experience in Ghana/Zambia (circle the appropriate one).

\section{Interview Sequence:}

1. Can you tell me a bit about how you came to go on a global health practicum?

a. Was it something that you had always planned on applying to participate in?

2. Can you tell me a little bit about this experience - what were some of the highlights for you?

3. Can you help me understand why these experiences were so rewarding for you?

I am hearing you say (reiterate the highlights/rewarding experiences perceived by the interviewee)....Now,

4. Were there any times when you were uncomfortable during your global health practicum?

a. How did you find yourself in this situation?

b. In what type of context did it arise?

c. Did you feel this sense of discomfort immediately? Or, did this feeling settle-in after a period of reflection?

5. Can you help me understand why you felt this feeling of discomfort?

a. What do you think was important to you in that experience?

b. What do you think was important to the people around you?

c. How did it make you feel?

6. What do you think you gained from participating in the GHP?

a. Do you think this is something you could have gained without engaging in a GHP?

b. Going into this experience, is this something that you thought you might gain? 
7. Now that you have had some time to reflect back on your GHP experience, do you think it is an activity that you would participate in again given the chance to do so?

a. If yes, why?

b. If no, what about your experience has left you questioning whether or not to engage in such an opportunity again?

As we're coming to the close of this interview, I would like to ask you if I were to say (reiterate some of the highlights of the GHP that the participant expressed), would that - in your mindrepresent what you felt about of the highlights of your experience? And if I were to say (reiterate some of the more troubling elements of the GHP that the participant expressed), would that represent some of the aspects that you found more troubling? And lastly, if I were to say (reiterate the participant's current reflections on their GHP experience), would that represent how you feel about your experience now?

We have come to the end of questions that I wanted to ask you. Have I missed anything that you think is relevant? Are there any questions that you would like to ask me?

Thank you so much for taking the time to chat with me today. If you think of a question, or something you would like to go over with me, please do not hesitate to contact me via e-mail or phone. My contact information is on the consent form that I will photocopy for you. 
Appendix G: Field Notes Template

THE U N IVER S I Y OF B R I T S H C O L U M B I A

Field Notes

Title of Project: The Ethical Implications of Global Health Practicums in Nursing

\section{Participant Code \#:}

Interview Date:

Starting Time:

Ending Time:

Location of interview:

People present:

Description of the environment:

Non-verbal behaviour: (e.g., tone of voice, posture, facial expressions, eye movements, forcefulness of speech, body movements, and hand gestures)

Content of interview: (e.g., use key words, topics, focus, exact words, or phases that stand out)

Researcher's impressions: (e.g., discomfort of participant with certain topics, emotional responses to events)

Preliminary Analysis: (e.g., researcher’s questions, tentative hunches, trends in data, and emerging patterns)

Technological problems: (e.g., lost 5 minutes of audio recording due to battery failure) 